\title{
Derivation of a one-dimensional von Kármán theory for viscoelastic ribbons
}

\author{
Manuel Friedrich® and Lennart Machillø
}

\begin{abstract}
We consider a two-dimensional model of viscoelastic von Kármán plates in the Kelvin's-Voigt's rheology derived from a three-dimensional model at a finite-strain setting in Friedrich and Kružík (Arch Ration Mech Anal 238: 489-540, 2020). As the width of the plate goes to zero, we perform a dimension-reduction from $2 \mathrm{D}$ to $1 \mathrm{D}$ and identify an effective one-dimensional model for a viscoelastic ribbon comprising stretching, bending, and twisting both in the elastic and the viscous stress. Our arguments rely on the abstract theory of gradient flows in metric spaces by Sandier and Serfaty (Commun Pure Appl Math 57:1627-1672, 2004) and complement the $\Gamma$-convergence analysis of elastic von Kármán ribbons in Freddi et al. (Meccanica 53:659-670, 2018). Besides convergence of the gradient flows, we also show convergence of associated time-discrete approximations, and we provide a corresponding commutativity result.
\end{abstract}

Mathematics Subject Classification. 74D10, 35A15, 35Q74, 49J45.

Keywords. Viscoelasticity, Metric gradient flows, Dimension reduction, $\Gamma$ convergence, Dissipative distance, Curves of maximal slope, Minimizing movements.

\section{Introduction}

The derivation of effective theories for thin structures such as plates, rods, or ribbons is a classical problem in continuum mechanics. Despite the long history of the subject with contributions already by Euler, Kirchhoff, and von Kármán (see $[6,12]$ for surveys), rigorous results relating lower-dimensional theories to three-dimensional elasticity have only been obtained comparably recently. They were triggered by the use of variational methods, particularly by $\Gamma$-convergence [13] together with quantitative rigidity estimates [24]. In this present work, we are interested in effective descriptions for viscoelastic ribbons, i.e., bodies with three different length scales: a length $l$ which is much larger than the width $\varepsilon$ which, in turn, is much larger than the thickness $h$. 
This difference in the characteristic dimensions allows to model the material effectively as a one-dimensional continuum [16].

In elasticity theory, the study of such models dates back to works by SADOWsky [26] and WunderLich [38] who proposed and formally justified one-dimensional energies from a two-dimensional Kirchhoff plate model, corresponding to $h=0$ and to the limiting passage $\varepsilon \rightarrow 0$. Recently, FreDDI, Hornung, MorA, AND PARONi [19] gave a rigorous justification of a corrected model and addressed also related effective descriptions derived from von Kármán plate models [20]. More generally, a hierarchy of one-dimensional models has been derived from three-dimensional nonlinear elasticity by considering the simultaneous limit $h \ll \varepsilon \rightarrow 0$ for appropriate rates $h / \varepsilon[17,18]$. On the contrary, the assumption $h \sim \varepsilon$ leads to different effective rod models identified in $[2,32,33]$.

The present work is devoted to a similar scenario of deriving onedimensional theories for viscoelastic von Kármán ribbons. Let us start by considering a nonlinear three-dimensional model of a viscoelastic material with reference configuration $\Omega_{\varepsilon, h}=\left(-\frac{l}{2}, \frac{l}{2}\right) \times\left(-\frac{\varepsilon}{2}, \frac{\varepsilon}{2}\right) \times\left(-\frac{h}{2}, \frac{h}{2}\right)$ at finite strains in Kelvin's-Voigt's rheology, i.e., a spring and a damper coupled in parallel. Neglecting inertia, the nonlinear system of equations takes the form

$$
-\operatorname{div}\left(\partial_{F} W(\nabla y)+\partial_{\dot{F}} R\left(\nabla y, \partial_{t} \nabla y\right)\right)=f \text { in }[0, T] \times \Omega_{\varepsilon, h},
$$

where $[0, T]$ is the process time interval and $y:[0, T] \times \Omega_{\varepsilon, h} \rightarrow \mathbb{R}^{3}$ denotes a deformation with gradient $\nabla y$. The tensors $\partial_{F} W$ and $\partial_{\dot{F}} R$ correspond to the elastic stress and the viscous stress, respectively, and are related to a stored energy density $W$ as well as a (pseudo-)potential $R$ of dissipative forces. (Here, $F$ and $\dot{F}$ are placeholders for $\nabla y$ and $\partial_{t} \nabla y$, respectively.) Eventually, $f$ describes an external force. In contrast to the rapidly developed static theory of nonlinear elasticity, due to the physically relevant assumptions on frame indifference for both $W$ and $R$ (see $[5,7]$ ), existence of solutions remains a challenging problem and results are scarce. We refer, e.g., to [28] for local intime existence or to [15] for the existence of measure-valued solutions. To date, weak solutions in a general finite strain setting [21,31] can only be guaranteed by using the concept of second-grade nonsimple materials where the elastic properties additionally depend on the second gradient of the deformation [39, 40]. At the same time, let us mention that a main justification of the model investigated in [21] lies in the observation that, in the small strain limit, the problem leads to the standard system of linear viscoelasticity without second gradient.

Recently in [22], starting from a version of (1.1) for nonsimple materials, a dimension reduction has been performed to derive a von Kármán-like viscoelastic plate model on a two-dimensional plate $S_{\varepsilon}=\left(-\frac{l}{2}, \frac{l}{2}\right) \times\left(-\frac{\varepsilon}{2}, \frac{\varepsilon}{2}\right)$. This complements the static $\Gamma$-convergence analysis for elastic materials [25], which has justified the von Kármán plate equations proposed more than 100 years ago [41]. In contrast to previous works on viscoelastic plates [9-11,35], where 
the starting point is already a plate model, [22] constitutes a rigorous derivation by proving that solutions to (1.1) converge in a suitable sense to effective two-dimensional equations. More precisely, there are in-plane displacements $u:[0, T] \times S_{\varepsilon} \rightarrow \mathbb{R}^{2}$ and out-of-plane displacements $v:[0, T] \times S_{\varepsilon} \rightarrow \mathbb{R}$ such that

$$
\left\{\begin{aligned}
g^{2 D}= & -\operatorname{div}\left(\mathbb{C}_{W}^{2}\left(e(u)+\frac{1}{2} \nabla v \otimes \nabla v\right)+\mathbb{C}_{R}^{2}\left(e\left(\partial_{t} u\right)+\nabla \partial_{t} v \otimes \nabla v\right)\right), \\
f^{2 D}= & -\operatorname{div}\left(\left(\mathbb{C}_{W}^{2}\left(e(u)+\frac{1}{2} \nabla v \otimes \nabla v\right)+\mathbb{C}_{R}^{2}\left(e\left(\partial_{t} u\right)+\nabla \partial_{t} v \otimes \nabla v\right)\right) \nabla v\right) \\
& +\frac{1}{12} \operatorname{div} \operatorname{div}\left(\mathbb{C}_{W}^{2} \nabla^{2} v+\mathbb{C}_{R}^{2} \nabla^{2} \partial_{t} v\right)
\end{aligned}\right.
$$

in $[0, T] \times S_{\varepsilon}$, where $e(u):=\frac{1}{2}\left(\nabla u+\nabla u^{T}\right)$ denotes the linear strain tensor, and $\mathbb{C}_{W}^{2}$ as well as $\mathbb{C}_{R}^{2}$ are tensors of elasticity and viscosity coefficients, respectively, derived suitably from $W$ and $R$. In addition to the vertical force $f^{2 D}$, we also consider a horizontal force $g^{2 D}$ that was not included in [22] for simplicity. The first equation features a membrane term both in the elastic and the viscous stress, and the second equation contains also a bending term. The problem is closely related to the von Kármán functional given by (neglecting the forces)

$$
\phi_{\varepsilon}(u, v):=\frac{1}{2} \int_{S_{\varepsilon}} Q_{W}^{2}\left(e(u)+\frac{1}{2} \nabla v \otimes \nabla v\right) \mathrm{d} x+\frac{1}{24} \int_{S_{\varepsilon}} Q_{W}^{2}\left(\nabla^{2} v\right) \mathrm{d} x,
$$

where $Q_{W}^{2}(F)=F: \mathbb{C}_{W}^{2} F$ for $F \in \mathbb{R}^{2 \times 2}$. Indeed, (1.2) can proved to be a (metric) gradient flow for $\phi_{\varepsilon}$ for a metric suitably related to $\mathbb{C}_{W}^{2}$, see again [22]. This approach was additionally exploited in [23] for numerical approximation of the system (1.2) via a minimizing movement scheme. We also refer to [1] for a related dynamical problem considering inertial terms but no viscosity.

In order to describe the one-dimensional effective behavior of thin viscoelastic ribbons, the goal of the present paper is to perform another dimension reduction by letting the width $\varepsilon$ in (1.2) go to zero. In a purely static setting, this problem has been addressed in [20] by identifying the $\Gamma$-limit of the sequence $\frac{1}{\varepsilon} \phi_{\varepsilon}$ in terms of the non-convex functional

$$
\begin{aligned}
\phi_{0}\left(\xi_{1}, \xi_{2}, w, \theta\right):= & \frac{1}{2} \int_{-l / 2}^{l / 2} Q_{W}^{0}\left(\xi_{1}^{\prime}+\frac{\left|w^{\prime}\right|^{2}}{2}\right) \mathrm{d} x_{1} \\
& +\frac{1}{24} \int_{-l / 2}^{l / 2}\left(Q_{W}^{0}\left(\xi_{2}^{\prime \prime}\right)+Q_{W}^{1}\left(w^{\prime \prime}, \theta^{\prime}\right)\right) \mathrm{d} x_{1}
\end{aligned}
$$

comprising stretching, bending, and twisting, where $Q_{W}^{0}$ and $Q_{W}^{1}$ are quadratic forms suitably related to $Q_{W}^{2}$. More precisely, the in-plane displacement $u$ can be related to an axial displacement $\xi_{1}$ and an orthogonal in-plane displacement $\xi_{2}$. In contrast, the out-of-plane displacement $v$ generates an out-of-plane displacement $w$, and the derivative of $v$ in the direction orthogonal to the axis leads to a twist function $\theta$. 
In the framework of viscoelastic ribbons, we relate the nonlinear equations (1.2) to the following equations for viscoelastic ribbons

$$
\left\{\begin{array}{l}
g_{1}^{1 D}=-\frac{\mathrm{d}}{\mathrm{d} x_{1}}\left(C_{W}^{0}\left(\xi_{1}^{\prime}+\frac{\left|w^{\prime}\right|^{2}}{2}\right)+C_{R}^{0}\left(\partial_{t} \xi_{1}^{\prime}+w^{\prime} \partial_{t} w^{\prime}\right)\right) \\
g_{2}^{1 D}=\frac{1}{12} \frac{\mathrm{d}^{2}}{\mathrm{~d} x_{1}^{2}}\left(C_{W}^{0} \xi_{2}^{\prime \prime}+C_{R}^{0} \partial_{t} \xi_{2}^{\prime \prime}\right) \\
f^{1 D}=-\frac{\mathrm{d}}{\mathrm{d} x_{1}}\left(\left(C_{W}^{0}\left(\xi_{1}^{\prime}+\frac{\left|w^{\prime}\right|^{2}}{2}\right)+C_{R}^{0}\left(\partial_{t} \xi_{1}^{\prime}+w^{\prime} \partial_{t} w^{\prime}\right)\right) w^{\prime}\right) \\
\quad+\frac{1}{24} \frac{\mathrm{d}^{2}}{\mathrm{~d} x_{1}^{2}}\left(\partial_{1} Q_{W}^{1}\left(w^{\prime \prime}, \theta^{\prime}\right)+\partial_{1} Q_{R}^{1}\left(\partial_{t} w^{\prime \prime}, \partial_{t} \theta^{\prime}\right)\right) \\
0=\frac{\mathrm{d}}{\mathrm{d} x_{1}}\left(\partial_{2} Q_{W}^{1}\left(w^{\prime \prime}, \theta^{\prime}\right)+\partial_{2} Q_{R}^{1}\left(\partial_{t} w^{\prime \prime}, \partial_{t} \theta^{\prime}\right)\right)
\end{array}\right.
$$

in $[0, T] \times\left(-\frac{l}{2}, \frac{l}{2}\right)$, where the constants $C_{W}^{0}>0$ and $C_{R}^{0}>0$, and the quadratic form $Q_{R}^{1}$ are again related to $Q_{W}^{2}$ and $Q_{R}^{2}$, respectively. Moreover, $f^{1 D}$, $g^{1 D}$ are forces derived from $f^{2 D}, g^{2 D}$. Note that the equations are again given in divergence form. More precisely, we prove existence of solutions to (1.4) and make the dimension reduction rigorous, i.e., we show that solutions to (1.2) converge to solutions of (1.4) in a specific sense. The solutions have to be understood in a weak sense, see (2.13) for the exact definition. The same property holds for time-discrete approximations, and we provide a corresponding commutativity result, see Fig. 1.

Heuristically, (1.4) can be understood as the effective equation of a thinwalled beam with reference configuration $\Omega_{\varepsilon, h}$ governed by (1.1), when we first let $h \rightarrow 0$ and then $\varepsilon \rightarrow 0$. In a forthcoming work, we will make this intuition rigorous by studying simultaneous limits $h \ll \varepsilon \rightarrow 0$, complementing the purely elastic analysis in [18]. Let us mention that in [20] also other energy regimes have been considered, leading to a "linearized" von Kármán or a "constrained" von Kármán energy. Whereas the former case is completely covered by our analysis, the latter is subtler due to the nonlinear constraint $\operatorname{det}\left(\nabla^{2} v\right)=0$ in the two-dimensional setting.

Our general strategy is to treat the systems (1.2) and (1.4) in the abstract setting of metric gradient flows [4] for the energies $\phi_{\varepsilon}$ and $\phi_{0}$, respectively, where the underlying metric is given by a dissipation distance suitably related to $\mathbb{C}_{R}^{2}, \partial_{1} Q_{R}^{1}, \partial_{2} Q_{R}^{1}$, and $C_{R}^{0}$. (We also refer to [30] for a thorough explanation to this approach.) We follow the approach of evolutionary $\Gamma$-convergence devised in $[29,34,36,37]$. In using this theory, the challenge lies in proving that the additional conditions needed to ensure convergence of gradient flows are satisfied.

More specifically, to use the abstract convergence result, lower semicontinuity of the energies, the metrics, and the local slopes is needed. The estimate for the energies and metrics essentially follows from [20], see Theorems 5.1 and 5.2 below. The lower semicontinuity of the local slopes, however, is more technical and the core of our argument. We briefly present the main idea in the one-dimensional setting. The local slope of $\phi_{0}$ in a metric space with metric 
$\mathcal{D}_{0}$ is defined by

$$
\left|\partial \phi_{0}\right|_{\mathcal{D}_{0}}(z):=\limsup _{\hat{z} \rightarrow z} \frac{\left(\phi_{0}(z)-\phi_{0}(\hat{z})\right)^{+}}{\mathcal{D}_{0}(z, \hat{z})} .
$$

(To simplify the notation here, we use a single variable $z$ in place of $\left(\xi_{1}, \xi_{2}, w, \theta\right)$, see (1.3).) We use a finer representation of the local slope, based on generalized convexity properties, to show that the local slope coincides with the global slope, see [4, Definition 1.2.4], up to some lower order terms. Essentially, this shows $\left|\partial \phi_{0}\right|_{\mathcal{D}_{0}}(z) \approx \frac{\left(\phi_{0}(z)-\phi_{0}(\tilde{z})\right)^{+}}{\mathcal{D}_{0}(z, \tilde{z})}$ for some $\tilde{z}$. Consider a sequence $\left(z_{n}\right)_{n \in \mathbb{N}}$ converging to a limit $z$. The main step consists in constructing a $m u$ tual recovery sequence $\left(\tilde{z}_{n}\right)_{n \in \mathbb{N}}$ such that $\phi_{0}\left(z_{n}\right)-\phi_{0}\left(\tilde{z}_{n}\right) \rightarrow \phi_{0}(z)-\phi_{0}(\tilde{z})$ and $\mathcal{D}_{0}\left(z_{n}, \tilde{z}_{n}\right) \rightarrow \mathcal{D}_{0}(z, \tilde{z})$, see Lemma A.2. The strategy to prove the lower semicontinuity of local slopes along the sequence $\phi_{\varepsilon}$ is similar, but the construction of mutual recovery sequences (see Lemma 5.3) is subtler as suitable compatibility conditions between the elastic energy and the viscous dissipation are needed. We consider different conditions in that direction, ranging from materials with small Poisson ratio to vanishing dissipation potentials in the direction of the width $\varepsilon$, see Subsection 2.3 for details. Let us emphasize that mutual recovery sequences are also crucial to perform the limiting passage on the time-discrete level, see Theorem 5.7.

The plan of the paper is as follows. In Sect. 2, we introduce the one- and two-dimensional models and state our main results. The main goal is to prove the existence of solutions to the one-dimensional model, which is based on gradient flows in metric spaces [4]. In particular, Theorem 2.2(i) provides the existence of solutions to the one-dimensional gradient flow by relying on the theory of generalized minimizing movements. Moreover, Theorem 2.2(ii) identifies solutions of the gradient flow as weak solutions of the one-dimensional system of PDEs (1.4). Finally, Theorem 2.3 addresses the relation to the twodimensional system. Besides the convergence of two-dimensional solutions to the one-dimensional solutions, we also get analogous results for the semidiscretized problems. In particular, convergences for vanishing time step and vanishing width of the plate commute, see Fig. 1. Section 3 is devoted to definitions concerning the theory of gradient flows in metric spaces. In particular, we recall the approximation scheme and also collect the necessary existence results for curves of maximal slopes $[4,14]$. While Sect. 4 collects separately the main properties of the two- and one-dimensional systems, Sect. 5 addresses the relation between the two systems. Finally, proofs of the main results can be found in Sect. 6, and several technical proofs are postponed to the Appendix A.

\section{Notation}

In what follows, we use standard notation for Lebesgue spaces, $L^{p}(\Omega)$, which are measurable maps on $\Omega \subset \mathbb{R}^{d}, d=1,2$, integrable with the $p$-th power (if $1 \leq p<+\infty$ ) or essentially bounded (if $p=+\infty$ ). Sobolev spaces, i.e., $W^{k, p}(\Omega)$ denote the linear spaces of maps which, together with their weak derivatives up to the order $k \in \mathbb{N}$, belong to $L^{p}(\Omega)$. Further, $W_{0}^{k, p}(\Omega)$ contains maps from $W^{k, p}(\Omega)$ having zero boundary conditions (in the sense of traces). 
Moreover, for a function $v \in W^{k, p}(\Omega)$, the set $W_{v}^{k, p}(\Omega)$ contains maps from $W^{k, p}(\Omega)$ attaining $v$ at the boundary (in the sense of traces) up to the $(k-1)$ th order. To emphasize the target space $E$, we write $W^{k, p}(\Omega ; E)$. If $E=\mathbb{R}$, we write $W^{k, p}(\Omega)$ as usual. We refer to [3] for more details on Sobolev spaces and their duals. If the integration variable is clear from the context, we usually drop $\mathrm{d} x$ at the end of integrals.

\section{The model and main results}

\subsection{The two-dimensional setting}

In this subsection we describe the two-dimensional von Kármán plate model. Fixing an interval $I=\left(-\frac{l}{2}, \frac{l}{2}\right)$, the set $S_{\varepsilon}:=I \times\left(-\frac{\varepsilon}{2}, \frac{\varepsilon}{2}\right)$ represents a twodimensional reference configuration of a two-dimensional plate, where $l>0$ denotes the length and $\varepsilon>0$ the width. For $u \in W^{1,2}\left(S_{\varepsilon} ; \mathbb{R}^{2}\right)$ and $v \in$ $W^{2,2}\left(S_{\varepsilon} ; \mathbb{R}\right)$ we define the von Kármán functional by

$$
\begin{aligned}
\phi_{\varepsilon}(u, v):= & \frac{1}{\varepsilon} \int_{S_{\varepsilon}} \frac{1}{2} Q_{W}^{2}\left(e(u)+\frac{1}{2} \nabla v \otimes \nabla v\right)+\frac{1}{\varepsilon} \int_{S_{\varepsilon}} \frac{1}{24} Q_{W}^{2}\left(\nabla^{2} v\right) \\
& -\frac{1}{\varepsilon} \int_{S_{\varepsilon}}\left(f_{\varepsilon}^{2 D} v+g_{\varepsilon}^{2 D} \cdot u\right),
\end{aligned}
$$

where $e(u):=\frac{1}{2}\left(\nabla u+\nabla u^{T}\right)$ denotes the linear strain tensor and $\otimes$ the Euclidean tensor product. Moreover, $Q_{W}^{2}$ denotes a quadratic form on $\mathbb{R}^{2 \times 2}$, related to the tensor $\mathbb{C}_{W}^{2}$ in (1.2) via the mapping $A \mapsto Q_{W}^{2}(A)=A: \mathbb{C}_{W}^{2} A$. The quadratic form is derived from a frame-indifferent energy density $W$. Therefore, it only depends on the symmetric part of a matrix $A \in \mathbb{R}^{2 \times 2}$, i.e., on $\frac{1}{2}\left(A+A^{T}\right)$, and is positive definite on $\mathbb{R}_{\mathrm{sym}}^{2 \times 2}$. This functional describes the energy of a two-dimensional plate deformed in three-dimensional space, where $u$ and $v$ correspond to in-plane and out-of-plane displacements, respectively. The energy comprises both a membrane term depending on $u$ and $v$, as well as a bending term, only depending on $v$. Eventually, the functions $f_{\varepsilon}^{2 D} \in L^{2}\left(S_{\varepsilon}\right)$ and $g_{\varepsilon}^{2 D} \in L^{2}\left(S_{\varepsilon} ; \mathbb{R}^{2}\right)$ correspond to a vertical force density and a horizontal force density, respectively.

We also introduce a global dissipation distance $\mathcal{D}_{\varepsilon}((u, v),(\tilde{u}, \tilde{v}))$ between two displacements $(u, v),(\tilde{u}, \tilde{v}) \in W^{1,2}\left(S_{\varepsilon} ; \mathbb{R}^{2}\right) \times W^{2,2}\left(S_{\varepsilon}\right)$ whose square is given by

$$
\begin{aligned}
\mathcal{D}_{\varepsilon}((u, v),(\tilde{u}, \tilde{v}))^{2}= & \frac{1}{\varepsilon} \int_{S_{\varepsilon}} Q_{R}^{2}\left(e(\tilde{u})-e(u)+\frac{1}{2} \nabla \tilde{v} \otimes \nabla \tilde{v}-\frac{1}{2} \nabla v \otimes \nabla v\right) \\
& +\frac{1}{12 \varepsilon} \int_{S_{\varepsilon}} Q_{R}^{2}\left(\nabla^{2} \tilde{v}-\nabla^{2} v\right) .
\end{aligned}
$$

Here, $Q_{R}^{2}$ is a quadratic form on $\mathbb{R}^{2 \times 2}$, positive definite on $\mathbb{R}_{\mathrm{sym}}^{2 \times 2}$, which corresponds to the tensor $\mathbb{C}_{R}^{2}$ in (1.2) and is derived from a nonlinear viscous dissipation potential $R$, see (1.1). 
As shown in $[22,23]$, the metric gradient flow of $\phi_{\varepsilon}$ with respect to the metric $\mathcal{D}_{\varepsilon}$, for a given initial datum $\left(u_{0}, v_{0}\right) \in W^{1,2}\left(S_{\varepsilon} ; \mathbb{R}^{2}\right) \times W^{2,2}\left(S_{\varepsilon}\right)$, corresponds to the $2 D$ equations for viscoelastic von Kármán plates

$$
\begin{cases}-\operatorname{div}\left(\mathbb{C}_{W}^{2}\left(e(u)+\frac{1}{2} \nabla v \otimes \nabla v\right)+\mathbb{C}_{R}^{2}\left(e\left(\partial_{t} u\right)+\nabla \partial_{t} v \odot \nabla v\right)\right)=g_{\varepsilon}^{2 D}, & \\ -\operatorname{div}\left(\left(\mathbb{C}_{W}^{2}\left(e(u)+\frac{1}{2} \nabla v \otimes \nabla v\right)+\mathbb{C}_{R}^{2}\left(e\left(\partial_{t} u\right)+\nabla \partial_{t} v \odot \nabla v\right)\right) \nabla v\right) & \\ \quad+\frac{1}{12} \operatorname{div} \operatorname{div}\left(\mathbb{C}_{W}^{2} \nabla^{2} v+\mathbb{C}_{R}^{2} \nabla^{2} \partial_{t} v\right)=f_{\varepsilon}^{2 D} & \text { in }[0, \infty) \times S_{\varepsilon}, \\ u(0, \cdot)=u_{0}, v(0, \cdot)=v_{0} & \text { in } S_{\varepsilon},\end{cases}
$$

where $\odot$ is the symmetrized tensor product and div denotes the distributional divergence. The existence of solutions to (2.3) complemented with Dirichlet boundary conditions on $\partial S_{\varepsilon}$ has been addressed in $[22,23]$. For performing the dimension reduction, we instead only impose boundary conditions on the lateral boundaries $\partial I \times\left(-\frac{\varepsilon}{2}, \frac{\varepsilon}{2}\right)$. More precisely, given functions $\hat{u}_{1} \in W^{1,2}(I)$, $\hat{u}_{2} \in W^{2,2}(I)$, and $\hat{v} \in W^{2,2}(I)$, we define the space of admissible functions by

$$
\begin{aligned}
\mathscr{S}_{\varepsilon}^{2 D}:= & \left\{(u, v) \in W^{1,2}\left(S_{\varepsilon} ; \mathbb{R}^{2}\right) \times W^{2,2}\left(S_{\varepsilon}\right):\right. \\
& u(x)=\left(\hat{u}_{1}\left(x_{1}\right)-x_{2} \hat{u}_{2}^{\prime}\left(x_{1}\right), \frac{1}{\varepsilon} \hat{u}_{2}\left(x_{1}\right)\right), v(x)=\hat{v}\left(x_{1}\right), \\
& \left.\partial_{1} v(x)=\partial_{1} \hat{v}\left(x_{1}\right) \text { on } \partial I \times\left(-\frac{\varepsilon}{2}, \frac{\varepsilon}{2}\right)\right\} .
\end{aligned}
$$

Note that the proof in $[22,23]$ can still be performed under $(2.4)$ up to minor adjustments, but the equations (2.3) need to be complemented with zero Neumann boundary conditions on the top and the bottom of $S_{\varepsilon}$. As we will see below, however, they do not affect the effective 1D model. The structure of the conditions on $u$ is related to the space of Bernoulli-Navier functions, see (2.9) below.

We say that $(u, v) \in W^{1,2}\left([0, \infty) ; \mathscr{S}_{\varepsilon}^{2 D}\right)$ is a weak solution of $(2.3)$ if $u(0, \cdot)=u_{0}, v(0, \cdot)=v_{0}$ and for a.e. $t \geq 0$ we have

$$
\begin{gathered}
\int_{S_{\varepsilon}}\left(\mathbb{C}_{W}^{2}\left(e(u)+\frac{1}{2} \nabla v \otimes \nabla v\right)+\mathbb{C}_{R}^{2}\left(e\left(\partial_{t} u\right)+\nabla \partial_{t} v \odot \nabla v\right)\right): \nabla \phi_{u}=\int_{S_{\varepsilon}} g_{\varepsilon}^{2 D} \cdot \phi_{u}, \\
\int_{S_{\varepsilon}}\left(\mathbb{C}_{W}^{2}\left(e(u)+\frac{1}{2} \nabla v \otimes \nabla v\right)+\mathbb{C}_{R}^{2}\left(e\left(\partial_{t} u\right)+\nabla \partial_{t} v \odot \nabla v\right)\right):\left(\nabla v \odot \nabla \phi_{v}\right) \\
+\frac{1}{12} \int_{S_{\varepsilon}}\left(\mathbb{C}_{W}^{2} \nabla^{2} v+\mathbb{C}_{R}^{2} \nabla^{2} \partial_{t} v\right): \nabla^{2} \phi_{v}=\int_{S_{\varepsilon}} f_{\varepsilon}^{2 D} \phi_{v}
\end{gathered}
$$

for all $\left(\phi_{u}, \phi_{v}\right) \in \mathscr{S}_{\varepsilon}^{0}$, where $\mathscr{S}_{\varepsilon}^{0}$ is defined as in (2.4) for $\hat{u}_{1}=\hat{u}_{2}=\hat{v}=0$. Note that $(2.5 \mathrm{a})$ corresponds to two scalar equations and $(2.5 \mathrm{~b})$ corresponds to one scalar equation, respectively.

\subsection{Compactness and limiting variables}

For the limiting passage, it is more convenient to work on a fixed domain that does not depend on $\varepsilon$. To this end, we introduce the set $S:=I \times\left(-\frac{1}{2}, \frac{1}{2}\right)$ and 
define the scaled versions $y \in W^{1,2}\left(S ; \mathbb{R}^{2}\right)$ and $w \in W^{2,2}(S)$ by $y_{1}\left(x_{1}, x_{2}\right):=u_{1}\left(x_{1}, \varepsilon x_{2}\right), \quad y_{2}\left(x_{1}, x_{2}\right):=\varepsilon u_{2}\left(x_{1}, \varepsilon x_{2}\right), \quad w\left(x_{1}, x_{2}\right):=v\left(x_{1}, \varepsilon x_{2}\right)$,

and the scaled differential operators $E^{\varepsilon}, \nabla_{\varepsilon}, \nabla_{\varepsilon}^{2}$ by

$$
\begin{aligned}
& E^{\varepsilon} y:=\left(\begin{array}{cc}
\partial_{1} y_{1} & \frac{1}{2 \varepsilon}\left(\partial_{1} y_{2}+\partial_{2} y_{1}\right) \\
\frac{1}{2 \varepsilon}\left(\partial_{2} y_{1}+\partial_{1} y_{2}\right) & \frac{1}{\varepsilon^{2}} \partial_{2} y_{2}
\end{array}\right), \\
& \nabla_{\varepsilon} w:=\left(\partial_{1} w, \frac{1}{\varepsilon} \partial_{2} w\right) \quad \text { and } \quad \nabla_{\varepsilon}^{2} w:=\left(\begin{array}{cc}
\partial_{11}^{2} w & \frac{1}{\varepsilon} \partial_{12}^{2} w \\
\frac{1}{\varepsilon} \partial_{21}^{2} w & \frac{1}{\varepsilon^{2}} \partial_{22}^{2} w
\end{array}\right) \text {. }
\end{aligned}
$$

Thus, by the chain rule we have

$E^{\varepsilon} y(x)=e(u)\left(x_{1}, \varepsilon x_{2}\right), \quad \nabla_{\varepsilon} w(x)=\nabla v\left(x_{1}, \varepsilon x_{2}\right), \quad \nabla_{\varepsilon}^{2} w(x)=\nabla^{2} v\left(x_{1}, \varepsilon x_{2}\right)$.

Similarly, we define the scaled forces $\hat{f}_{\varepsilon}^{2 D}: S \rightarrow \mathbb{R}$ and $\hat{g}_{\varepsilon}^{2 D}: S \rightarrow \mathbb{R}^{2}$ by $\hat{f}_{\varepsilon}^{2 D}\left(x_{1}, x_{2}\right)=f_{\varepsilon}^{2 D}\left(x_{1}, \varepsilon x_{2}\right)$ and $\hat{g}_{\varepsilon}^{2 D}\left(x_{1}, x_{2}\right)=g_{\varepsilon}^{2 D}\left(x_{1}, \varepsilon x_{2}\right)$ and assume that the scaled versions satisfy

$$
\hat{f}_{\varepsilon}^{2 D} \rightarrow f^{1 D}, \quad \hat{g}_{\varepsilon, 1}^{2 D} \rightarrow g_{1}^{1 D}, \quad \frac{1}{\varepsilon} \hat{g}_{\varepsilon, 2}^{2 D} \rightarrow g_{2}^{1 D} \quad \text { weakly in } L^{2}(S)
$$

for functions $f^{1 D} \in L^{2}(I)$ and $g^{1 D} \in L^{2}\left(I ; \mathbb{R}^{2}\right)$. Here and in the following, with a slight abuse of notation, we regard all functions defined on $I$ as functions on $S$ which do not depend explicitly on the variable $x_{2}$.

Starting from the variables $y$ and $w$ in dimension two, we now introduce corresponding limiting variables in the one-dimensional setting. We will identify the limit of the in-plane displacements with two-dimensional BernoulliNavier functions defined by

$$
\begin{gathered}
B N_{\left(\hat{u}_{1}, \hat{u}_{2}\right)}\left(S ; \mathbb{R}^{2}\right):=\left\{y \in W^{1,2}\left(S ; \mathbb{R}^{2}\right): e(y)_{12}=e(y)_{22}=0, \quad y_{1}=\hat{u}_{1}-x_{2} \hat{u}_{2}^{\prime},\right. \\
\left.y_{2}=\hat{u}_{2} \text { on } \Gamma\right\}
\end{gathered}
$$

where for shorthand, we set $\Gamma:=\partial I \times\left(-\frac{1}{2}, \frac{1}{2}\right)$. Compared to [20], this function space is different since in our analysis we consider functions with boundary values instead of functions with vanishing mean. By arguing analogously to $[8$, Theorem 4.1], the space of Bernoulli-Navier functions can be identified with functions defined on $I$, namely

$$
\begin{gathered}
B N_{\left(\hat{u}_{1}, \hat{u}_{2}\right)}\left(S ; \mathbb{R}^{2}\right)=\left\{y \in W^{1,2}\left(S ; \mathbb{R}^{2}\right): \exists \xi_{1} \in W_{\hat{u}_{1}}^{1,2}(I), \exists \xi_{2} \in W_{\hat{u}_{2}}^{2,2}(I)\right. \text { such that } \\
\left.y_{1}(x)=\xi_{1}\left(x_{1}\right)-x_{2} \xi_{2}^{\prime}\left(x_{1}\right), \quad y_{2}(x)=\xi_{2}\left(x_{1}\right)\right\} .
\end{gathered}
$$

Here, it is worth noting that the second component has a higher regularity and that $\xi_{2}^{\prime}=\hat{u}_{2}^{\prime}$ on $\partial I$ as $\xi_{1}-x_{2} \xi_{2}^{\prime}=\hat{u}_{1}-x_{2} \hat{u}_{2}^{\prime}$ on $\partial I$ for a.e. $x_{2} \in\left(-\frac{1}{2}, \frac{1}{2}\right)$. We recall the scalings $(2.6)$ and $(2.7)$, and denote by $\mathscr{S}_{\varepsilon, M}^{2 D}:=\{(u, v) \in$ $\left.\mathscr{S}_{\varepsilon}^{2 D}: \phi_{\varepsilon}(u, v) \leq M\right\}$ the sublevel sets of the energy.

Proposition 2.1. (Compactness) Let $\left(u_{\varepsilon}, v_{\varepsilon}\right)_{\varepsilon}$ be a sequence such that $\left(u_{\varepsilon}, v_{\varepsilon}\right) \in$ $\mathscr{S}_{\varepsilon, M}^{2 D}$ for $M>0$. Let $\left(y_{\varepsilon}, w_{\varepsilon}\right)_{\varepsilon}$ be the scaled sequence in the sense of (2.6). Then, up to a subsequence, there exists a vertical displacement $w \in W_{\hat{v}}^{2,2}(I)$, a 
twist function $\theta \in W_{0}^{1,2}(I)$, and a horizontal displacement $y \in B N_{\left(\hat{u}_{1}, \hat{u}_{2}\right)}\left(S ; \mathbb{R}^{2}\right)$ such that

$$
\begin{aligned}
& w_{\varepsilon} \rightarrow w \text { in } W^{2,2}(S), \quad \nabla_{\varepsilon} w_{\varepsilon} \rightarrow\left(w^{\prime}, \theta\right) \text { in } W^{1,2}\left(S ; \mathbb{R}^{2}\right), \\
& \nabla_{\varepsilon}^{2} w_{\varepsilon} \rightarrow\left(\begin{array}{cc}
w^{\prime \prime} & \theta^{\prime} \\
\theta^{\prime} & \gamma
\end{array}\right) \text { in } L^{2}\left(S ; \mathbb{R}_{\mathrm{sym}}^{2 \times 2}\right)
\end{aligned}
$$

for a suitable $\gamma \in L^{2}(S)$ and

$$
y_{\varepsilon} \rightarrow y \text { in } W^{1,2}\left(S ; \mathbb{R}^{2}\right) \quad \text { and } \quad E^{\varepsilon} y_{\varepsilon} \rightarrow E \text { in } L^{2}\left(S ; \mathbb{R}_{\mathrm{sym}}^{2 \times 2}\right)
$$

for a suitable $E \in L^{2}\left(S ; \mathbb{R}_{\mathrm{sym}}^{2 \times 2}\right)$ such that $E_{11}=\partial_{1} y_{1}$.

The proof is omitted as it closely follows the lines of [20, Lemma 2.1] where functions with vanishing mean have been considered. In fact, the only difference lies in using suitable versions of Poincaré's and Korn's inequality for functions with given trace, and in checking that the limits satisfy the boundary conditions. To see the latter, it suffices to observe that $y_{\varepsilon}(x)=\left(\hat{u}_{1}\left(x_{1}\right)-\right.$ $\left.x_{2} \hat{u}_{2}^{\prime}\left(x_{1}\right), \hat{u}_{2}\left(x_{1}\right)\right), w_{\varepsilon}(x)=\hat{v}\left(x_{1}\right)$, and $\frac{1}{\varepsilon} \partial_{2} w_{\varepsilon}=0$ on $\Gamma$, see (2.4) and (2.6). Here, our choice of the boundary values in (2.4) becomes apparent since it guarantees that the limit of the in-plane displacements can be identified with functions in (2.9). We also refer to [27], where clamped boundary conditions in a related context are considered. Later we will see that the compactness result also holds in the time-dependent setting along solutions to (2.5).

\subsection{Effective quadratic forms and compatibility conditions}

As a preparation for the formulation of the one-dimensional model, we introduce effective quadratic forms related to $Q_{S}^{2}, S=W, R$, introduced in Subsection 2.1. Recall that $Q_{W}^{2}$ and $Q_{R}^{2}$ are defined on $\mathbb{R}^{2 \times 2}$ which depend only on symmetric matrices and can thus be identified with functions defined on $\mathbb{R}^{3}$ via $\left(q_{11}, q_{12}, q_{22}\right) \simeq\left(\begin{array}{ll}q_{11} & q_{12} \\ q_{12} & q_{22}\end{array}\right)$. For the sake of readability, we use both types of notation in the sequel. We define reduced quadratic forms by minimizing the second and third entry. More precisely, we let

$$
Q_{S}^{1}\left(q_{11}, q_{12}\right):=\min _{\alpha \in \mathbb{R}} Q_{S}^{2}\left(q_{11}, q_{12}, \alpha\right)
$$

for $\left(q_{11}, q_{12}\right) \in \mathbb{R}^{2}$ and

$$
Q_{S}^{0}\left(q_{11}\right):=\min _{z \in \mathbb{R}} Q_{S}^{1}\left(q_{11}, z\right)=C_{S}^{0} q_{11}^{2}
$$

for $q_{11} \in \mathbb{R}$ and a suitable constant $C_{S}^{0}>0$. We denote by $\mathbb{C}_{W}^{1}$ and $\mathbb{C}_{R}^{1}$ the corresponding second-order tensors.

To perform a rigorous evolutionary dimension reduction, we require some compatibility conditions of the quadratic forms $Q_{W}^{2}$ and $Q_{R}^{2}$ as we need to construct mutual recovery sequences, compatible for the elastic energy and the viscous dissipation at the same time (see Theorems 5.6 and 5.7). A first possibility is given by the assumption that

$$
\underset{\alpha \in \mathbb{R}}{\arg \min } Q_{S}^{2}\left(q_{11}, q_{12}, \alpha\right)=0, \quad \underset{z \in \mathbb{R}}{\arg \min } Q_{S}^{1}\left(q_{11}, z\right)=0 \quad \text { for all } q_{11}, q_{12} \in \mathbb{R} \text {. }
$$


This induces a restriction from a modeling point of view since it particularly corresponds to materials with Poisson ratio zero, such as cork. A reasonable generalization is to assume that $Q_{S}^{2}$ are $\varepsilon$-dependent, denoted by $Q_{S, \varepsilon}^{2}$, such that $Q_{S, \varepsilon}^{2}=Q_{S}^{2}+\mathrm{o}(1) \hat{Q}_{S}$ for $\varepsilon \rightarrow 0$, where $Q_{S}^{2}$ satisfies $(\mathbf{H 1})$ and $\hat{Q}_{S}$ is any positive definite quadratic form. (For simplicity, we did not include this explicitly in the notation in (2.1) and (2.2).) Another sound option is to consider thin materials with general Poisson ratio, but with a vanishing dissipation effect in the $e_{2}$ direction. In this case, the assumptions are given by

$$
\lim _{\varepsilon \rightarrow 0} Q_{R, \varepsilon}^{2}\left(q_{11}, q_{12}, \alpha\right)=Q_{R}^{1}\left(q_{11}, q_{12}\right), \quad \underset{z \in \mathbb{R}}{\arg \min } Q_{S}^{1}\left(q_{11}, z\right)=0 \text { for } S=W, R
$$

for all $q_{11}, q_{12}, \alpha \in \mathbb{R}$. This setting includes, but is not restricted to, the case of linear isotropic elastic materials with corresponding quadratic form

$$
Q_{W}^{2}\left(q_{11}, q_{12}, q_{22}\right):=2 \mu\left(q_{11}^{2}+2 q_{12}^{2}+q_{22}^{2}\right)+\lambda\left(q_{11}+q_{22}\right)^{2},
$$

where $\mu>0$ and $\lambda \in \mathbb{R}$ are suitable Lamé parameters. In particular, (H1) corresponds to $\lambda=0$. In this paper, we cover both cases described above. We remark that $(\mathbf{H 1})$ and $(\mathbf{H 2})$ are only needed for the proof of Lemma 5.3.

\subsection{Equations of viscoelastic vK ribbons in 1D}

We now present the effective $1 \mathrm{D}$ equations. To this end, define the set of admissible functions by

$$
\mathcal{K}:=W_{\hat{u}_{1}}^{1,2}(I) \times W_{\hat{u}_{2}}^{2,2}(I) \times W_{\hat{v}}^{2,2}(I) \times W_{0}^{1,2}(I) .
$$

In the following, we write ' for spatial- and $\partial_{t}$ for time derivatives. Recall the definition of the forces in (2.8). Given $\left(\xi_{1}^{0}, \xi_{2}^{0}(t), w^{0}, \theta^{0}\right) \in \mathcal{K}$, we consider the system of equations

$$
\begin{aligned}
g_{1}^{1 D}= & -\frac{\mathrm{d}}{\mathrm{d} x_{1}}\left(C_{W}^{0}\left(\xi_{1}^{\prime}+\frac{\left|w^{\prime}\right|^{2}}{2}\right)+C_{R}^{0}\left(\partial_{t} \xi_{1}^{\prime}+w^{\prime} \partial_{t} w^{\prime}\right)\right), \\
g_{2}^{1 D}= & \frac{1}{12} \frac{\mathrm{d}^{2}}{\mathrm{~d} x_{1}^{2}}\left(C_{W}^{0} \xi_{2}^{\prime \prime}+C_{R}^{0} \partial_{t} \xi_{2}^{\prime \prime}\right), \\
f^{1 D}= & -\frac{\mathrm{d}}{\mathrm{d} x_{1}}\left(\left(C_{W}^{0}\left(\xi_{1}^{\prime}+\frac{\left|w^{\prime}\right|^{2}}{2}\right)+C_{R}^{0}\left(\partial_{t} \xi_{1}^{\prime}+w^{\prime} \partial_{t} w^{\prime}\right)\right) w^{\prime}\right) \\
& +\frac{1}{24} \frac{\mathrm{d}^{2}}{\mathrm{~d} x_{1}^{2}}\left(\partial_{1} Q_{W}^{1}\left(w^{\prime \prime}, \theta^{\prime}\right)+\partial_{1} Q_{R}^{1}\left(\partial_{t} w^{\prime \prime}, \partial_{t} \theta^{\prime}\right)\right), \\
0= & \frac{\mathrm{d}}{\mathrm{d} x_{1}}\left(\partial_{2} Q_{W}^{1}\left(w^{\prime \prime}, \theta^{\prime}\right)+\partial_{2} Q_{R}^{1}\left(\partial_{t} w^{\prime \prime}, \partial_{t} \theta^{\prime}\right)\right)
\end{aligned}
$$$$
\text { in }[0, \infty) \times I
$$

such that $\xi_{1}(0, \cdot)=\xi_{1}^{0}, \xi_{2}(0, \cdot)=\xi_{2}^{0}, w(0, \cdot)=w^{0}, \theta(0, \cdot)=\theta^{0}$ in $I$ and $\left(\xi_{1}(t), \xi_{2}(t), w(t), \theta(t)\right) \in \mathcal{K}$ for $t \in[0, \infty)$. We also say that $\left(\xi_{1}, \xi_{2}, w, \theta\right) \in$ $W^{1,2}([0, \infty) ; \mathcal{K})$ is a weak solution if $\xi_{1}(0, \cdot)=\xi_{1}^{0}, \xi_{2}(0, \cdot)=\xi_{2}^{0}, w(0, \cdot)=w^{0}$, $\theta(0, \cdot)=\theta^{0}$ and for a.e. $t \geq 0$, we have

$$
0=\int_{I}\left(C_{W}^{0}\left(\xi_{1}^{\prime}+\frac{\left|w^{\prime}\right|^{2}}{2}\right)+C_{R}^{0}\left(\partial_{t} \xi_{1}^{\prime}+w^{\prime} \partial_{t} w^{\prime}\right)\right) \phi_{\xi_{1}}^{\prime}-\int_{I} g_{1}^{1 D} \phi_{\xi_{1}},
$$




$$
\begin{aligned}
0= & \int_{I} \frac{1}{12}\left(C_{W}^{0} \xi_{2}^{\prime \prime}+C_{R}^{0} \partial_{t} \xi_{2}^{\prime \prime}\right) \phi_{\xi_{2}}^{\prime \prime}-\int_{I} g_{2}^{1 D} \phi_{\xi_{2}}, \\
0= & \int_{I}\left(C_{W}^{0}\left(\xi_{1}^{\prime}+\frac{\left|w^{\prime}\right|^{2}}{2}\right)+C_{R}^{0}\left(\partial_{t} \xi_{1}^{\prime}+w^{\prime} \partial_{t} w^{\prime}\right)\right) w^{\prime} \phi_{w}^{\prime} \\
& +\frac{1}{24} \int_{I}\left(\partial_{1} Q_{W}^{1}\left(w^{\prime \prime}, \theta^{\prime}\right)+\partial_{1} Q_{R}^{1}\left(\partial_{t} w^{\prime \prime}, \partial_{t} \theta^{\prime}\right)\right) \phi_{w}^{\prime \prime}-\int_{I} f^{1 D} \phi_{w}, \\
0= & \int_{I}\left(\partial_{2} Q_{W}^{1}\left(w^{\prime \prime}, \theta^{\prime}\right)+\partial_{2} Q_{R}^{1}\left(\partial_{t} w^{\prime \prime}, \partial_{t} \theta^{\prime}\right)\right) \phi_{\theta}^{\prime}
\end{aligned}
$$

for all $\phi_{\xi_{1}} \in W_{0}^{1,2}(I), \phi_{\xi_{2}} \in W_{0}^{2,2}(I), \phi_{w} \in W_{0}^{2,2}(I)$, and $\phi_{\theta} \in W_{0}^{1,2}(I)$.

We point out that $(2.13 \mathrm{~b})$ describing the orthogonal in-plane displacement $\xi_{2}$ is completely decoupled from the other equations, whereas the axial in-plane displacement $\xi_{1}$ is always coupled to the vertical displacement $w$ by (2.13a) and (2.13c). Interestingly, under assumption (H1), one can check that the twist function only appears in $(2.13 \mathrm{~d})$, and $(2.13 \mathrm{~d})$ is also independent of $w$ in this setting, i.e., (2.13d) decouples completely, as well.

Our goal will be to show existence of weak solutions to (2.13) and that weak solutions (2.5) converge to weak solutions (2.13) in a suitable sense. In particular, we will relate (2.13) to a metric gradient flow with respect to an energy $\phi_{0}$ in the space

$$
\mathscr{S}^{1 D}:=B N_{\left(\hat{u}_{1}, \hat{u}_{2}\right)}\left(S ; \mathbb{R}^{2}\right) \times W_{\hat{v}}^{2,2}(I) \times W_{0}^{1,2}(I),
$$

endowed with a metric $\mathcal{D}_{0}$ whose square is given by

$$
\begin{aligned}
\mathcal{D}_{0}((y, w, \theta),(\tilde{y}, \tilde{w}, \tilde{\theta}))^{2}:= & \int_{S} Q_{R}^{0}\left(\partial_{1} y_{1}-\partial_{1} \tilde{y}_{1}+\frac{\left|w^{\prime}\right|^{2}}{2}-\frac{\left|\tilde{w}^{\prime}\right|^{2}}{2}\right) \\
& +\frac{1}{12} \int_{I} Q_{R}^{1}\left(w^{\prime \prime}-\tilde{w}^{\prime \prime}, \theta^{\prime}-\tilde{\theta}^{\prime}\right)
\end{aligned}
$$

for $(y, w, \theta),(\tilde{y}, \tilde{w}, \tilde{\theta}) \in \mathscr{S}^{1 D}$ and the energy $\phi_{0}$ is given by

$$
\phi_{0}(y, w, \theta):=\frac{1}{2} \int_{S} Q_{W}^{0}\left(\partial_{1} y_{1}+\frac{\left|w^{\prime}\right|^{2}}{2}\right)+\frac{1}{24} \int_{I} Q_{W}^{1}\left(w^{\prime \prime}, \theta^{\prime}\right)-\int_{I} f^{1 D} w+g^{1 D} \cdot y
$$

for $(y, w, \theta) \in \mathscr{S}^{1 D}$. Note that (2.16) coincides with (1.3) (for $f^{1 D}=0, g^{1 D}=$ 0 ) by using (2.9) and by performing an integration over $x_{2}$, where one uses $\int_{-1 / 2}^{1 / 2} x_{2}=0$ and $\int_{-1 / 2}^{1 / 2} x_{2}^{2}=1 / 12$. For notational convenience, we work with $\mathscr{S}^{1 D}$ instead of the (equivalent) space $\mathcal{K}$, i.e., we identify $\left(\xi_{1}, \xi_{2}\right)$ with $y$ via $(2.9)$.

\subsection{Main results}

To show existence and convergence of solutions, we will use the abstract theory of gradient flows [4] and evolutionary $\Gamma$-convergence $[29,36,37]$. In particular, our approach to prove existence of $1 \mathrm{D}$ solutions is twofold as we derive it both by time-discrete approximations and also by limits of two-dimensional solutions. 
Our first main result addresses the existence of time-discrete solutions to the one-dimensional problem and their convergence to a curve of maximal slope for $\phi_{0}$ with respect to $\mathcal{D}_{0}$ and $\left|\partial \phi_{0}\right|_{\mathcal{D}_{0}}$. For the main definitions and notation for curves of maximal slope and strong upper gradients we refer to Subsection 3.1. In particular, we write $\left|\partial \phi_{\varepsilon}\right|_{\mathcal{D}_{\varepsilon}}$ and $\left|\partial \phi_{0}\right|_{\mathcal{D}_{0}}$ for the local slopes, where the energies and metrics are defined in (2.1), (2.2), (2.15), and (2.16). The definition of time-discrete solutions is given in Subsection 3.2. The relevant results about existence of curves of maximal slope are recalled in Subsections 3.2 and 3.3.

Theorem 2.2. (Solutions in the one-dimensional setting) Consider $\left(\xi_{1}^{0}, \xi_{2}^{0}, w^{0}, \theta^{0}\right) \in$ $\mathcal{K}$ and define $y^{0}:=\left(\xi_{1}^{0}-x_{2}\left(\xi_{2}^{0}\right)^{\prime}, \xi_{2}^{0}\right)$, i.e., $\left(y^{0}, w^{0}, \theta^{0}\right) \in \mathscr{S}^{1 D}$.

(i) (Approximation and existence) For each null sequence $\left(\tau_{l}\right)_{l \in \mathbb{N}}$ and each sequence of discrete solutions $\left(\bar{U}_{\tau_{l}}\right)_{l}$ as in (3.2) below, there exists an absolutely continuous function $(y, w, \theta):[0, \infty) \rightarrow \mathscr{S}^{1 D}$ with respect to the metric $\mathcal{D}_{0}$ satisfying $(y, w, \theta)(0)=\left(y^{0}, w^{0}, \theta^{0}\right)$ such that, up to a subsequence, not relabeled,

$$
\bar{U}_{\tau_{l}}(t) \rightarrow(y(t), w(t), \theta(t)) \quad \text { for all } t \in[0, \infty) \text { as } l \rightarrow \infty
$$

with respect to the topology induced by $\mathcal{D}_{0}$, and $(y, w, \theta)$ is a curve of maximal slope for $\phi_{0}$ with respect to $\left|\partial \phi_{0}\right|_{\mathcal{D}_{0}}$.

(ii) (Identification) Each curve of maximal slope $(y, w, \theta)$ for $\phi_{0}$ with respect to $\left|\partial \phi_{0}\right|_{\mathcal{D}_{0}}$ can be identified via (2.9) with a curve

$$
\left(\xi_{1}, \xi_{2}, w, \theta\right) \in W^{1,2}([0, \infty) ; \mathcal{K})
$$

such that $\left(\xi_{1}, \xi_{2}, w, \theta\right)$ is a weak solution of the system (2.13).

Now, we study the relation of weak solutions (2.5) and weak solutions (2.13). To this end, we need to specify the topology of the convergence. We define mappings $\pi_{\varepsilon}: \mathscr{S}_{\varepsilon}^{2 D} \rightarrow \mathscr{S}$ by $\pi_{\varepsilon}(u, v):=\left(y, w, \frac{\partial_{2} w}{\varepsilon}\right)$, where $y$ and $w$ are the scaled in-plane and out-of-plane displacements corresponding to $u$ and $v$, see (2.6), and $\mathscr{S}=\pi_{\varepsilon}\left(\mathscr{S}_{\varepsilon}^{2 D}\right)$. We say that $\pi_{\varepsilon}\left(u_{\varepsilon}, v_{\varepsilon}\right)=\left(y_{\varepsilon}, w_{\varepsilon}, \frac{\partial_{2} w_{\varepsilon}}{\varepsilon}\right) \stackrel{\sigma}{\rightarrow}(y, w, \theta)$ if we have the convergence in the sense of Proposition 2.1. Furthermore, we say that $\left(u_{\varepsilon}, v_{\varepsilon}\right) \stackrel{\pi \sigma}{\rightarrow}(y, w, \theta)$ if $\pi_{\varepsilon}\left(u_{\varepsilon}, v_{\varepsilon}\right) \stackrel{\sigma}{\rightarrow}(y, w, \theta)$. The sequence $\left(u_{\varepsilon}, v_{\varepsilon}\right)_{\varepsilon}$ converges strongly to $(y, w, \theta)$, written $\left(u_{\varepsilon}, v_{\varepsilon}\right) \stackrel{\pi \rho}{\rightarrow}(y, w, \theta)$, if the convergence in Proposition 2.1 also holds with respect to the strong in place of the weak topology. We remark that the limiting variables $(y, w, \theta)$ are contained in the space $\mathscr{S}^{1 D} \subset \mathscr{S}$ defined in (2.14).

Theorem 2.3. (Relation between two-dimensional and one-dimensional system) Suppose that (H1) or (H2) holds. Consider a null sequence $\left(\varepsilon_{l}\right)_{l \in \mathbb{N}}$ and a sequence of initial data $\left(u_{\varepsilon_{l}}^{0}, v_{\varepsilon_{l}}^{0}\right)_{\varepsilon_{l}}$ with $\left(u_{\varepsilon_{l}}^{0}, v_{\varepsilon_{l}}^{0}\right) \in \mathscr{S}_{\varepsilon_{l}, M}^{2 D}$ such that $\left(u_{\varepsilon_{l}}^{0}, v_{\varepsilon_{l}}^{0}\right) \stackrel{\pi \sigma}{\rightarrow}\left(y^{0}, w^{0}, \theta^{0}\right) \in \mathscr{S}^{1 D}$ and $\phi_{\varepsilon}\left(u_{\varepsilon_{l}}^{0}, v_{\varepsilon_{l}}^{0}\right) \rightarrow \phi_{0}\left(y^{0}, w^{0}, \theta^{0}\right)$.

(i) (Convergence of continuous solutions) Let $\left(u_{\varepsilon_{l}}, v_{\varepsilon_{l}}\right)_{l}$ be a sequence of curves of maximal slopes for $\phi_{\varepsilon_{l}}$ with respect to $\left|\partial \phi_{\varepsilon_{l}}\right|_{\mathcal{D}_{\varepsilon_{l}}}$ satisfying $\left(u_{\varepsilon_{l}}(0), v_{\varepsilon_{l}}(0)\right)=\left(u_{\varepsilon_{l}}^{0}, v_{\varepsilon_{l}}^{0}\right)$. Then, there exists an absolutely continuous 


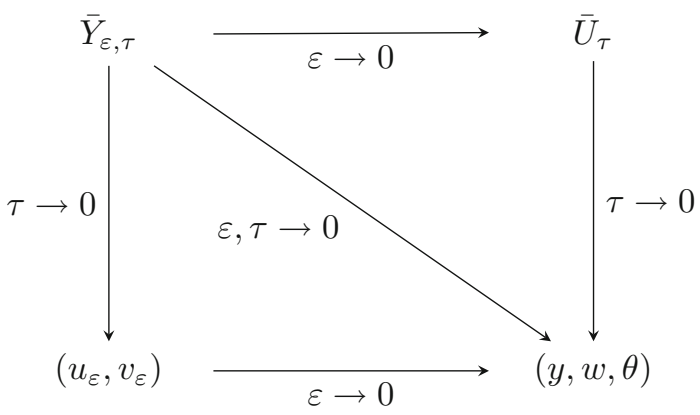

FIGURE 1. Illustration of the commutativity result given in Theorems 2.2 and 2.3, where $\tau$ indicates the timestep and $\varepsilon$ the width of the ribbon. The horizontal and diagonal arrows are addressed in Theorem 2.3. The left vertical arrow is a consequence of [23, Theorem 4.1], up to minor adaptions due to boundary conditions. The remaining vertical arrow corresponds to Theorem 2.2

function $(y, w, \theta):[0, \infty) \rightarrow \mathscr{S}^{1 D}$ with respect to the metric $\mathcal{D}_{0}$ satisfying $(y, w, \theta)(0)=\left(y^{0}, w^{0}, \theta^{0}\right)$ such that, up to a subsequence, not relabeled,

$$
\left(u_{\varepsilon_{l}}(t), v_{\varepsilon_{l}}(t)\right) \stackrel{\pi \rho}{\rightarrow}(y(t), w(t), \theta(t)) \quad \text { for all } t \in[0, \infty) \text { as } l \rightarrow \infty
$$

and $(y, w, \theta)$ is a curve of maximal slope for $\phi_{0}$ with respect to $\left|\partial \phi_{0}\right|_{\mathcal{D}_{0}}$.

(ii) (Convergence of discrete solutions) For all $\tau>0$ and all discrete solutions $\bar{Y}_{\varepsilon_{l}, \tau}$ as in (3.2) below there exists a discrete solution $\bar{U}_{\tau}$ of the onedimensional system such that, up to a subsequence, not relabeled,

$$
\bar{Y}_{\varepsilon_{l}, \tau} \stackrel{\pi \rho}{\rightarrow} \bar{U}_{\tau} \quad \text { for all } t \in[0, \infty) \text { as } l \rightarrow \infty .
$$

(iii) (Convergence at specific scales) For each null sequence $\left(\tau_{l}\right)_{l \in \mathbb{N}}$ and each sequence of discrete solutions $\bar{Y}_{\varepsilon_{l}, \tau_{l}}$ as in (3.2) below, there exists an absolutely continuous function $(y, w, \theta):[0, \infty) \rightarrow \mathscr{S}^{1 D}$ with respect to the metric $\mathcal{D}_{0}$ satisfying $(y, w, \theta)(0)=\left(y^{0}, w^{0}, \theta^{0}\right)$ such that, up to a subsequence, not relabeled,

$$
\bar{Y}_{\varepsilon_{l}, \tau_{l}}(t) \stackrel{\pi \rho}{\rightarrow}(y(t), w(t), \theta(t)) \quad \text { for all } t \in[0, \infty) \text { as } l \rightarrow \infty
$$

and $(y, w, \theta)$ is a curve of maximal slope for $\phi_{0}$ with respect to $\left|\partial \phi_{0}\right|_{\mathcal{D}_{0}}$.

Note that in the two-dimensional setting the existence of curves of maximal slope in (i) and discrete solutions in (ii) and (iii) is guaranteed by [22, Theorem 2.2] and [23, Theorem 4.1]. In particular, weak solutions in the twodimensional setting converge (in the sense above) to solutions of the onedimensional equations. We refer to Fig. 1 for an illustration. We point out that existence of solutions to the one-dimensional equations follows without (H1) or (H2), see Theorem 2.2. These compatibility conditions are only needed to prove Theorem 2.3. 


\section{Preliminaries: curves of maximal slope}

In this section, we recall the relevant definitions about curves of maximal slope and present abstract theorems concerning the convergence of time-discrete solutions and continuous solutions to curves of maximal slope.

\subsection{Definitions}

We consider a complete metric space $(\mathscr{S}, \mathcal{D})$. We say a curve $y:(a, b) \rightarrow \mathscr{S}$ is absolutely continuous with respect to $\mathcal{D}$ if there exists $m \in L^{1}(a, b)$ such that

$$
\mathcal{D}(y(s), y(t)) \leq \int_{s}^{t} m(r) \mathrm{d} r \quad \text { for all } a \leq s \leq t \leq b .
$$

The smallest function $m$ with this property, denoted by $\left|y^{\prime}\right|_{\mathcal{D}}$, is called metric derivative of $y$ and satisfies for a.e. $t \in(a, b)$ (see [4, Theorem 1.1.2] for the existence proof)

$$
\left|y^{\prime}\right|_{\mathcal{D}}(t):=\lim _{s \rightarrow t} \frac{\mathcal{D}(y(s), y(t))}{|s-t|} .
$$

We define the notion of a curve of maximal slope. We only give the basic definition here and refer to $[4$, Section $1.2,1.3]$ for motivations and more details. By $h^{+}:=\max (h, 0)$ we denote the positive part of a function $h$.

Definition 3.1. (Upper gradients, slopes, curves of maximal slope) We consider a complete metric space $(\mathscr{S}, \mathcal{D})$ with a functional $\phi: \mathscr{S} \rightarrow(-\infty,+\infty]$.

(i) A function $g: \mathscr{S} \rightarrow[0, \infty]$ is called a strong upper gradient for $\phi$ if for every absolutely continuous curve $y:(a, b) \rightarrow \mathscr{S}$ the function $g \circ y$ is Borel and

$$
|\phi(y(t))-\phi(y(s))| \leq \int_{s}^{t} g(y(r))\left|y^{\prime}\right|_{\mathcal{D}}(r) \mathrm{d} r \quad \text { for all } a<s \leq t<b .
$$

(ii) For each $y \in \mathscr{S}$ the local slope of $\phi$ at $y$ is defined by

$$
|\partial \phi|_{\mathcal{D}}(y):=\limsup _{z \rightarrow y} \frac{(\phi(y)-\phi(z))^{+}}{\mathcal{D}(y, z)} .
$$

(iii) An absolutely continuous curve $y:(a, b) \rightarrow \mathscr{S}$ is called a curve of maximal slope for $\phi$ with respect to the strong upper gradient $g$ if for a.e. $t \in(a, b)$

$$
\frac{\mathrm{d}}{\mathrm{d} t} \phi(y(t)) \leq-\frac{1}{2}\left|y^{\prime}\right|_{\mathcal{D}}^{2}(t)-\frac{1}{2} g^{2}(y(t)) .
$$

\subsection{Curves of maximal slope as limits of time-discrete solutions}

We consider a sequence of complete metric spaces $\left(\mathscr{S}_{k}, \mathcal{D}_{k}\right)_{k}$, as well as a limiting complete metric space $\left(\mathscr{S}_{0}, \mathcal{D}_{0}\right)$. Moreover, let $\left(\phi_{k}\right)_{k}$ be a sequence of functionals with $\phi_{k}: \mathscr{S}_{k} \rightarrow[0, \infty]$ and $\phi_{0}: \mathscr{S}_{0} \rightarrow[0, \infty]$.

We introduce time-discrete solutions for the energy $\phi_{k}$ and the metric $\mathcal{D}_{k}$ by solving suitable time-incremental minimization problems: consider a fixed 
time step $\tau>0$ and suppose that an initial datum $Y_{k, \tau}^{0}$ is given. Whenever $Y_{k, \tau}^{0}, \ldots, Y_{k, \tau}^{n-1}$ are known, $Y_{k, \tau}^{n}$ is defined as (if existent)

$$
Y_{k, \tau}^{n}=\operatorname{argmin}_{v \in \mathscr{S}_{k}} \boldsymbol{\Phi}_{\mathbf{k}}\left(\tau, Y_{k, \tau}^{n-1} ; v\right), \quad \mathbf{\Phi}_{\mathbf{k}}(\tau, u ; v):=\frac{1}{2 \tau} \mathcal{D}_{k}(v, u)^{2}+\phi_{k}(v) .
$$

We suppose that for a choice of $\tau$ a sequence $\left(Y_{k, \tau}^{n}\right)_{n \in \mathbb{N}}$ solving (3.1) exists. Then we define the piecewise constant interpolation by

$$
\bar{Y}_{k, \tau}(0)=Y_{k, \tau}^{0}, \quad \bar{Y}_{k, \tau}(t)=Y_{k, \tau}^{n} \text { for } t \in((n-1) \tau, n \tau], n \geq 1 .
$$

We call $\bar{Y}_{k, \tau}$ a time-discrete solution. Note that the existence of such solutions is usually guaranteed by the direct method of the calculus of variations under suitable compactness, coercivity, and lower semicontinuity assumptions.

Our goal is to study the limit of time-discrete solutions as $k \rightarrow \infty$. To this end, we need to introduce a suitable topology for the convergence. We suppose that there exists a set $\mathscr{S}$ with $\mathscr{S} \supseteq \mathscr{S}_{0}$ and a projection $\pi_{k}: \mathscr{S}_{k} \rightarrow \mathscr{S}$. (Note that a usual assumption is $\mathscr{S}=\mathscr{S}_{0}$, see e.g. [37], but for our application we need a slightly more general setting.)

We assume that there is a possibly weaker topology $\sigma$ on $\mathscr{S}$. Given a sequence $\left(z_{k}\right)_{k}, z_{k} \in \mathscr{S}_{k}$, and $z \in \mathscr{S}$, we say $z_{k} \stackrel{\pi \sigma}{\rightarrow} z$ if $\pi_{k}\left(z_{k}\right) \stackrel{\sigma}{\rightarrow} z$. We suppose that the topology $\sigma$ satisfies

$$
z_{k} \stackrel{\pi \sigma}{\rightarrow} z, \quad \bar{z}_{k} \stackrel{\pi \sigma}{\rightarrow} \bar{z} \Rightarrow \liminf _{k \rightarrow \infty} \mathcal{D}_{k}\left(z_{k}, \bar{z}_{k}\right) \geq \mathcal{D}_{0}(z, \bar{z})
$$

for all $z, \bar{z} \in \mathscr{S}_{0}$. Moreover, we assume that for every sequence $\left(z_{k}\right)_{k}, z_{k} \in \mathscr{S}_{k}$, and $N \in \mathbb{N}$ we have

$$
\phi_{k}\left(z_{k}\right) \leq N \quad \Rightarrow \quad z_{k} \stackrel{\pi \sigma}{\rightarrow} z \in \mathscr{S}_{0} \quad \text { (up to a subsequence). }
$$

Further, we suppose lower semicontinuity of the energies and the slopes in the following sense: for all $z \in \mathscr{S}_{0}$ and $\left(z_{k}\right)_{k}, z_{k} \in \mathscr{S}_{k}$, we have

$$
z_{k} \stackrel{\pi \sigma}{\rightarrow} z \quad \Rightarrow \quad \liminf _{k \rightarrow \infty}\left|\partial \phi_{k}\right|_{\mathcal{D}_{k}}\left(z_{k}\right) \geq\left|\partial \phi_{0}\right|_{\mathcal{D}_{0}}(z), \quad \liminf _{k \rightarrow \infty} \phi_{k}\left(z_{k}\right) \geq \phi_{0}(z) .
$$

We now formulate the main convergence result of time-discrete solutions to curves of maximal slope, proved in [34, Section 2].

Theorem 3.2. Suppose that (3.3)-(3.5) hold. Moreover, assume that $\left|\partial \phi_{0}\right|_{\mathcal{D}_{0}}$ is a strong upper gradient for $\phi_{0}$. Consider a null sequence $\left(\tau_{k}\right)_{k}$. Let $\left(Y_{k, \tau_{k}}^{0}\right)_{k}$ with $Y_{k, \tau_{k}}^{0} \in \mathscr{S}_{k}$ and $\bar{z}_{0} \in \mathscr{S}_{0}$ be initial data satisfying

$$
Y_{k, \tau_{k}}^{0} \stackrel{\pi \sigma}{\rightarrow} \bar{z}_{0}, \quad \phi_{k}\left(Y_{k, \tau_{k}}^{0}\right) \rightarrow \phi_{0}\left(\bar{z}_{0}\right) .
$$

Then, for each sequence of discrete solutions $\left(\bar{Y}_{k, \tau_{k}}\right)_{k}$ starting from $\left(Y_{k, \tau_{k}}^{0}\right)_{k}$, there exists a limiting function $z:[0,+\infty) \rightarrow \mathscr{S}_{0}$ such that, up to a subsequence, not relabeled,

$$
\bar{Y}_{k, \tau_{k}}(t) \stackrel{\pi \sigma}{\rightarrow} z(t), \quad \phi_{k}\left(\bar{Y}_{k, \tau_{k}}(t)\right) \rightarrow \phi_{0}(z(t)) \quad \forall t \geq 0
$$


as $k \rightarrow \infty$, and $z$ is a curve of maximal slope for $\phi_{0}$ with respect to $\left|\partial \phi_{0}\right|_{\mathcal{D}_{0}}$. In particular, $z$ satisfies the energy identity

$$
\frac{1}{2} \int_{0}^{T}\left|z^{\prime}\right|_{\mathcal{D}_{0}}^{2}(t) \mathrm{d} t+\frac{1}{2} \int_{0}^{T}\left|\partial \phi_{0}\right|_{\mathcal{D}_{0}}^{2}(z(t)) \mathrm{d} t+\phi_{0}(z(T))=\phi_{0}\left(\bar{z}_{0}\right) \quad \forall T>0 .
$$

The statement is a combination of convergence results for curves of maximal slope [37] with their approximation by time-discrete solutions via the minimizing movement scheme. For a more detailed discussion of similar statements, we refer for example to [22, Section 3].

\subsection{Curves of maximal slope as limits of continuous solutions}

As before, $\left(\mathscr{S}_{k}, \mathcal{D}_{k}\right)_{k}$ and $\left(\mathscr{S}_{0}, \mathcal{D}_{0}\right)$ denote complete metric spaces, with corresponding functionals $\left(\phi_{k}\right)_{k}$ and $\phi_{0}$. For the relation of the two- and onedimensional systems, we will use the following result.

Theorem 3.3. Suppose that (3.3)-(3.5) hold. Moreover, assume that $\left|\partial \phi_{n}\right|_{\mathcal{D}_{n}}$, $\left|\partial \phi_{0}\right|_{\mathcal{D}_{0}}$ are strong upper gradients for $\phi_{n}, \phi_{0}$ with respect to $\mathcal{D}_{n}, \mathcal{D}_{0}$, respectively. Let $\bar{u} \in \mathscr{S}_{0}$. For all $n \in \mathbb{N}$, let $u_{n}$ be a curve of maximal slope for $\phi_{n}$ with respect to $\left|\partial \phi_{n}\right|_{\mathcal{D}_{n}}$ such that

$$
\begin{aligned}
& \text { (i) } \sup _{n \in \mathbb{N}} \sup _{t \geq 0} \phi_{n}\left(u_{n}(t)\right)<\infty \\
& \text { (ii) } u_{n}(0) \stackrel{\pi \sigma}{\longrightarrow} \bar{u}, \quad \phi_{n}\left(u_{n}(0)\right) \rightarrow \phi_{0}(\bar{u}) .
\end{aligned}
$$

Then, there exists a limiting function $u:[0, \infty) \rightarrow \mathscr{S}_{0}$ such that up to a subsequence, not relabeled,

$$
u_{n}(t) \stackrel{\pi \sigma}{\rightarrow} u(t), \quad \phi_{n}\left(u_{n}(t)\right) \rightarrow \phi_{0}(u(t)) \quad \forall t \geq 0
$$

as $n \rightarrow \infty$ and $u$ is a curve of maximal slope for $\phi_{0}$ with respect to $\left|\partial \phi_{0}\right|_{\mathcal{D}_{0}}$.

The result is a variant of [37] and is given in [21, Theorem 3.6], with the only difference being that here we consider a sequence of spaces instead of a fixed space. The generalization is straightforward and follows from standard adaptions.

\section{Properties of energies and dissipation distances}

In this section, we collect basic properties of the energies and dissipation distances, and we establish properties for the local slopes. We recall the definition of the energy and the dissipation distance in (2.1), (2.16) and (2.2), (2.15), respectively. We also recall the notation for the sublevel sets $\mathscr{S}_{\varepsilon, M}^{2 D}=\{(u, v) \in$ $\left.\mathscr{S}_{\varepsilon}^{2 D}: \phi_{\varepsilon}(u, v) \leq M\right\}$. In what follows, we assume that $f^{2 D}, g^{2 D}=0$ for the sake of simplicity, see (2.1). Indeed, the force terms can be included in the analysis by minor, standard modifications. 


\subsection{Properties in 2D}

In this subsection, we state the relevant properties of the local slopes in the two-dimensional setting, which are provided by the following lemma.

Lemma 4.1. (Properties of the two-dimensional setting) Let $M>0$. We have:

(i) $\left(\mathscr{S}_{\varepsilon, M}^{2 D}, \mathcal{D}_{\varepsilon}\right)$ is a complete metric space.

(ii) Let $\Phi^{1}(t):=\sqrt{t^{2}+C t^{3}+C t^{4}}$ and $\Phi_{M}^{2}(t):=C \sqrt{M} t^{2}+C t^{3}+C t^{4}$ for any $C>0$ large enough. Suppose that $(u, v) \in \mathscr{S}_{\varepsilon, M}^{2 D}$. Then, the local slope for the energy $\phi_{\varepsilon}$ admits the representation

$$
\left|\partial \phi_{\varepsilon}\right|_{\mathcal{D}_{\varepsilon}}(u, v)=\sup _{\substack{(\tilde{u}, \tilde{v}) \in \mathscr{S}^{2 D} \\(u, v) \neq(\tilde{u}, \tilde{v})}} \frac{\left(\phi_{\varepsilon}(u, v)-\phi_{\varepsilon}(\tilde{u}, \tilde{v})-\Phi_{M}^{2}\left(\mathcal{D}_{\varepsilon}((u, v),(\tilde{u}, \tilde{v}))\right)\right)^{+}}{\Phi^{1}\left(\mathcal{D}_{\varepsilon}((u, v),(\tilde{u}, \tilde{v}))\right)} .
$$

(iii) The local slope $\left|\partial \phi_{\varepsilon}\right|_{\mathcal{D}_{\varepsilon}}$ is a strong upper gradient for $\phi_{\varepsilon}$.

Proof. Item (i) is proved in [22, Lemma 4.6]. For the proof of (ii), we refer to $[22$, Lemmas 4.8 and 4.9]. One only needs to ensure that the constant $C$ can be chosen independently of $\varepsilon$. The crucial step is the scaling of the embedding $W^{1,2}\left(S_{\varepsilon}\right) \subset \subset L^{4}\left(S_{\varepsilon}\right)$ on the thin domain $S_{\varepsilon}$ in order to deal with the nonlinearity $\nabla v \otimes \nabla v$ in the energy (2.1) and the metric (2.2). More precisely, by a scaling argument, $[22,(4.15)]$ can be replaced by

$$
\begin{aligned}
\left\|\frac{1}{2} \nabla\left(v_{0}-v_{1}\right) \otimes \nabla\left(v_{0}-v_{1}\right)\right\|_{L^{2}\left(S_{\varepsilon}\right)} & \leq C\left\|\nabla\left(v_{0}-v_{1}\right)\right\|_{L^{4}\left(S_{\varepsilon}\right)}^{2} \\
& \leq C \varepsilon^{-1 / 2}\left\|v_{0}-v_{1}\right\|_{W^{2,2}\left(S_{\varepsilon}\right)}^{2} \\
& \leq C \sqrt{\varepsilon} \mathcal{D}_{\varepsilon}^{2}\left(\left(u_{0}, v_{0}\right),\left(u_{1}, v_{1}\right)\right)
\end{aligned}
$$

for $\left(u_{0}, v_{0}\right),\left(u_{1}, v_{1}\right) \in \mathscr{S}_{\varepsilon, M}^{2 D}$, where the last inequality follows from $(2.2)$, Poincaré's inequality, and the positivity of $Q_{R}^{2}$. This is sufficient to adapt the proof of [22, Lemma 4.8]. The mappings $\Phi^{1}$ and $\Phi_{M}^{2}$ have been introduced after $[22,(4.19)]$ and before $[22,(4.21)]$, respectively. Item (iii) is also a consequence of [22, Lemma 4.9].

\subsection{Properties in $1 \mathrm{D}$}

In this subsection, we derive properties in the one-dimensional setting. We mainly need a one-dimensional version of Lemma 4.1 as well as some basic properties of the metric space $\left(\mathscr{S}^{1 D}, \mathcal{D}_{0}\right)$ and the energy $\phi_{0}$. The proofs are analogous to the corresponding proofs in [21,22]. We refer to Appendix A where we give the main arguments for the reader's convenience.

Lemma 4.2. (Properties of $\left(\mathscr{S}^{1 D}, \mathcal{D}_{0}\right)$ and $\left.\phi_{0}\right)$ Consider the canonical norm $\|\cdot\|_{\text {can }}$ on $\mathscr{S}^{1 D}$ which is defined as

$$
\|(y, w, \theta)\|_{c a n}:=\|y\|_{W^{1,2}\left(S ; \mathbb{R}^{2}\right)}+\|w\|_{W^{2,2}(I)}+\|\theta\|_{W^{1,2}(I)} .
$$

(i) Completeness: $\left(\mathscr{S}^{1 D}, \mathcal{D}_{0}\right)$ is a complete metric space.

(ii) Topology: The topology induced by $\|\cdot\|_{\text {can }}$ coincides with the topology induced by $\mathcal{D}_{0}$. In particular, there exists a constant $C>0$ such that

$\|w-\tilde{w}\|_{W^{2,2}(I)}+\|\theta-\tilde{\theta}\|_{W^{1,2}(I)} \leq C \mathcal{D}_{0}((y, w, \theta),(\tilde{y}, \tilde{w}, \tilde{\theta})) \quad$ and 


$$
\|y-\tilde{y}\|_{W^{1,2}\left(S ; \mathbb{R}^{2}\right)} \leq C \mathcal{D}_{0}((y, w, \theta),(\tilde{y}, \tilde{w}, \tilde{\theta}))+C\left\|w^{\prime}+\tilde{w}^{\prime}\right\|_{L^{4}(I)}\left\|w^{\prime}-\tilde{w}^{\prime}\right\|_{L^{4}(I)}
$$

for $(y, w, \theta),(\tilde{y}, \tilde{w}, \tilde{\theta}) \in \mathscr{S}^{1 D}$.

(iii) Compactness: Let $\left(y_{k}, w_{k}, \theta_{k}\right)_{k}$ be a sequence in $\mathscr{S}^{1 D}$ with $\sup _{k \in \mathbb{N}}$ $\phi_{0}\left(y_{k}, w_{k}, \theta_{k}\right)<\infty$. Then, $\sup _{k \in \mathbb{N}}\left\|\left(y_{k}, w_{k}, \theta_{k}\right)\right\|_{c a n}<+\infty$ and, up to a subsequence, $\left(y_{k}, w_{k}, \theta_{k}\right)_{k}$ converges weakly in $\left(\mathscr{S}^{1 D},\|\cdot\|_{\text {can }}\right)$ to some $(y, w, \theta) \in \mathscr{S}^{1 D}$.

(iv) Lower semicontinuity: If $\left(y_{k}, w_{k}, \theta_{k}\right)$ and $\left(\tilde{y}_{k}, \tilde{w}_{k}, \tilde{\theta}_{k}\right)$ converge weakly in $\left(\mathscr{S}^{1 D},\|\cdot\|_{\text {can }}\right)$ to $(y, w, \theta)$ and $(\tilde{y}, \tilde{w}, \tilde{\theta})$, respectively, we have $\liminf _{k \rightarrow \infty}$ $\phi_{0}\left(y_{k}, w_{k}, \theta_{k}\right) \geq \phi_{0}(y, w, \theta)$ and $\liminf _{k \rightarrow \infty} \mathcal{D}_{0}\left(\left(y_{k}, w_{k}, \theta_{k}\right),\left(\tilde{y}_{k}, \tilde{w}_{k}, \tilde{\theta}_{k}\right)\right) \geq$ $\mathcal{D}_{0}((y, w, \theta),(\tilde{y}, \tilde{w}, \tilde{\theta}))$.

The following lemma is the one-dimensional version of Lemma 4.1.

Lemma 4.3. (Properties of the one-dimensional slope $\left.\left|\partial \phi_{0}\right|_{\mathcal{D}_{0}}\right)$ Let $M>0$. We have:

(i) The local slope for the energy $\phi_{0}$ admits the representation

$\left|\partial \phi_{0}\right|_{\mathcal{D}_{0}}(y, w, \theta)$

$$
=\sup _{(y, w, \theta) \neq(\tilde{y}, \tilde{w}, \tilde{\theta}) \in \mathscr{S}^{1 D}} \frac{\left(\phi_{0}(y, w, \theta)-\phi_{0}(\tilde{y}, \tilde{w}, \tilde{\theta})-\Phi_{M}^{2}\left(\mathcal{D}_{0}((y, w, \theta),(\tilde{y}, \tilde{w}, \tilde{\theta}))\right)\right)^{+}}{\Phi^{1}\left(\mathcal{D}_{0}((y, w, \theta),(\tilde{y}, \tilde{w}, \tilde{\theta}))\right)}
$$

for all $(y, w, \theta) \in \mathscr{S}^{1 D}$ satisfying $\phi_{0}(y, w, \theta) \leq M$, where $\Phi^{1}$ and $\Phi_{M}^{2}$ are defined in Lemma 4.1(ii).

(ii) The local slope $\left|\partial \phi_{0}\right|_{\mathcal{D}_{0}}$ is a strong upper gradient for $\phi_{0}$.

(iii) Lower semicontinuity: If $\left(y_{k}, w_{k}, \theta_{k}\right)_{k} \subset \mathscr{S}^{1 D}$ converges weakly in $\left(\mathscr{S}^{1 D}, \|\right.$. $\left.\|_{\text {can }}\right)$ to $(y, w, \theta) \in \mathscr{S}^{1 D}$, we have $\liminf _{k \rightarrow \infty}\left|\partial \phi_{0}\right|_{\mathcal{D}_{0}}\left(y_{k}, w_{k}, \theta_{k}\right) \geq$ $\left|\partial \phi_{0}\right|_{\mathcal{D}_{0}}(y, w, \theta)$.

\section{Relation between $2 \mathrm{D}$ and $1 \mathrm{D}$ setting}

In this section, we briefly recall the convergence results in the static case [20] and then we prove lower semicontinuity for the local slopes along the passage from the $2 \mathrm{D}$ to the $1 \mathrm{D}$ setting. Recall the projection mapping $\pi_{\varepsilon}$, the space $\mathscr{S}=\pi_{\varepsilon}\left(\mathscr{S}_{\varepsilon}^{2 D}\right)$, and the convergences $\pi \sigma$ and $\pi \rho$ introduced before Theorem 2.3. In the sequel, it is convenient to express $\phi_{\varepsilon}$ and $\mathcal{D}_{\varepsilon}$ in terms of the scaled functions $y$ and $w$ introduced in (2.6). By a change of variables we have (recall $\left.f^{2 D}=g^{2 D}=0\right)$

$$
\phi_{\varepsilon}(y, w)=\frac{1}{2} \int_{S} Q_{W}^{2}\left(E^{\varepsilon} y+\frac{1}{2} \nabla_{\varepsilon} w \otimes \nabla_{\varepsilon} w\right)+\frac{1}{24} \int_{S} Q_{W}^{2}\left(\nabla_{\varepsilon}^{2} w\right)
$$

and

$$
\begin{aligned}
\mathcal{D}_{\varepsilon}^{2}((y, w),(\tilde{y}, \tilde{w}))= & \int_{S} Q_{R}^{2}\left(E^{\varepsilon} y-E^{\varepsilon} \tilde{y}+\frac{1}{2} \nabla_{\varepsilon} w \otimes \nabla_{\varepsilon} w-\frac{1}{2} \nabla_{\varepsilon} \tilde{w} \otimes \nabla_{\varepsilon} \tilde{w}\right) \\
& +\frac{1}{12} \int_{S} Q_{R}^{2}\left(\nabla_{\varepsilon}^{2} w-\nabla_{\varepsilon}^{2} \tilde{w}\right)
\end{aligned}
$$

for all $(y, w),(\tilde{y}, \tilde{w}) \in \mathscr{S}$. 


\section{1. $\Gamma$-convergence}

We briefly recall the $\Gamma$-convergence result in [20] which particularly yields the lower semicontinuity of the energies and the dissipation.

Theorem 5.1. ( $\Gamma$-convergence of energies) $\phi_{\varepsilon}$ converges to $\phi_{0}$ in the sense of $\Gamma$-convergence. More precisely,

(i) (Lower bound) For all $(y, w, \theta) \in \mathscr{S}^{1 D}$ and all sequences $\left(u_{\varepsilon}, v_{\varepsilon}\right)_{\varepsilon},\left(u_{\varepsilon}, v_{\varepsilon}\right) \in$ $\mathscr{S}_{\varepsilon}^{2 D}$, such that $\left(u_{\varepsilon}, v_{\varepsilon}\right) \stackrel{\pi \sigma}{\longrightarrow}(y, w, \theta)$ we find

$$
\liminf _{\varepsilon \rightarrow 0} \phi_{\varepsilon}\left(u_{\varepsilon}, v_{\varepsilon}\right) \geq \phi_{0}(y, w, \theta) .
$$

(ii) (Optimality of lower bound) For all $(y, w, \theta) \in \mathscr{S}^{1 D}$ there exists a sequence $\left(u_{\varepsilon}, v_{\varepsilon}\right)_{\varepsilon}$ with $\left(u_{\varepsilon}, v_{\varepsilon}\right) \in \mathscr{S}_{\varepsilon}^{2 D}$ for all $\varepsilon>0$ such that $\left(u_{\varepsilon}, v_{\varepsilon}\right) \stackrel{\pi \rho}{\longrightarrow}(y, w, \theta)$ and

$$
\lim _{\varepsilon \rightarrow 0} \phi_{\varepsilon}\left(u_{\varepsilon}, v_{\varepsilon}\right)=\phi_{0}(y, w, \theta) \text {. }
$$

In [20, Theorem 2.3(i),(ii)], the proof has been given for functions with vanishing mean. The adaptions to the present setting, however, are minor. In particular, the construction of recovery sequences needs to be slightly adjusted to comply with the imposed boundary conditions. We defer details to Lemma 5.3 and Remark 5.5(a) below where we use a similar ansatz as in [20].

Theorem 5.2. (Lower semicontinuity of dissipation distances) Let $M>0$. Then, for sequences $\left(u_{\varepsilon}, v_{\varepsilon}\right)_{\varepsilon}$ and $\left(\tilde{u}_{\varepsilon}, \tilde{v}_{\varepsilon}\right)_{\varepsilon},\left(u_{\varepsilon}, v_{\varepsilon}\right),\left(\tilde{u}_{\varepsilon}, \tilde{v}_{\varepsilon}\right) \in \mathscr{S}_{\varepsilon, M}^{2 D}$, with $\left(u_{\varepsilon}, v_{\varepsilon}\right) \stackrel{\pi \sigma}{\longrightarrow}(y, w, \theta)$ and $\left(\tilde{u}_{\varepsilon}, \tilde{v}_{\varepsilon}\right) \stackrel{\pi \sigma}{\longrightarrow}(\tilde{y}, \tilde{w}, \tilde{\theta})$ we have

$$
\liminf _{\varepsilon \rightarrow 0} \mathcal{D}_{\varepsilon}\left(\left(u_{\varepsilon}, v_{\varepsilon}\right),\left(\tilde{u}_{\varepsilon}, \tilde{v}_{\varepsilon}\right)\right) \geq \mathcal{D}_{0}((y, w, \theta),(\tilde{y}, \tilde{w}, \tilde{\theta})) .
$$

Proof. The argument is analogous to the one in Theorem 5.1(i) (cf. [20, Theorem 2.3(i)]) as the structure of $\mathcal{D}_{\varepsilon}$ and $\mathcal{D}_{0}$ is similar to $\phi_{\varepsilon}$ and $\phi_{0}$, see (2.15), (2.16), (5.1), and (5.2).

\subsection{Lower semicontinuity of slopes}

In contrast to Theorems 5.1 and 5.2, the derivation of lower semicontinuity for the local slopes is more challenging as in its definitions metrics appear in the denominator and energies are subtracted in the enumerator. Roughly speaking, we need a reverse inequality in Theorem 5.1(i) and Theorem 5.2 which in general is false. The representations of the slopes in Lemmas 4.1 and 4.3 allow us to construct recovery sequences such that the "reverse inequality" is true. More precisely, we have the following.

Lemma 5.3. (Mutual recovery sequence) Suppose that (H1) or (H2) holds. Consider a sequence $\left(u_{\varepsilon}, v_{\varepsilon}\right)_{\varepsilon},\left(u_{\varepsilon}, v_{\varepsilon}\right) \in \mathscr{S}_{\varepsilon, M}^{2 D}$, such that $\left(u_{\varepsilon}, v_{\varepsilon}\right) \stackrel{\pi \sigma}{\rightarrow}(y, w, \theta)$. Let $(\tilde{y}, \tilde{w}, \tilde{\theta}) \in \mathscr{S}^{1 D}$. Then, there exists a mutual recovery sequence $\left(\tilde{u}_{\varepsilon}, \tilde{v}_{\varepsilon}\right)_{\varepsilon}$, $\left(\tilde{u}_{\varepsilon}, \tilde{v}_{\varepsilon}\right) \in \mathscr{S}_{\varepsilon}^{2 D}$ for all $\varepsilon>0$, such that

$$
\liminf _{\varepsilon \rightarrow 0}\left(\phi_{\varepsilon}\left(u_{\varepsilon}, v_{\varepsilon}\right)-\phi_{\varepsilon}\left(\tilde{u}_{\varepsilon}, \tilde{v}_{\varepsilon}\right)\right) \geq \phi_{0}(y, w, \theta)-\phi_{0}(\tilde{y}, \tilde{w}, \tilde{\theta})
$$

and

$$
\lim _{\varepsilon \rightarrow 0} \mathcal{D}_{\varepsilon}\left(\left(u_{\varepsilon}, v_{\varepsilon}\right),\left(\tilde{u}_{\varepsilon}, \tilde{v}_{\varepsilon}\right)\right)=\mathcal{D}_{0}((y, w, \theta),(\tilde{y}, \tilde{w}, \tilde{\theta}))
$$


Remark 5.4. In the following and in later proofs, we will frequently use the elementary expansion

$$
Q(a)-Q(b)=Q(a-b)+2 \mathbb{C}[a-b, b]
$$

for all $a, b$, where $Q$ is a quadratic form with associated bilinear form $\mathbb{C}$.

Proof of Lemma 5.3. The proof is based on the ansatz of the recovery sequence in [20], slightly modified to comply with the imposed boundary conditions. Recall the definition of the quadratic forms in (2.10)-(2.11). We first suppose that (H2) holds as this case is more delicate. At the end of the proof, we briefly present the adaptions for $(\mathbf{H} \mathbf{1})$.

Step 1: Definition of recovery sequences. Given $\left(u_{\varepsilon}, v_{\varepsilon}\right)$, let $\left(y_{\varepsilon}, w_{\varepsilon}\right)$ as in (2.6). Let $\gamma, E_{12}$, and $E_{22}$ be the functions given by Proposition 2.1 such that $\frac{1}{\varepsilon^{2}} \partial_{22} w_{\varepsilon} \rightarrow \gamma,\left(E^{\varepsilon} y_{\varepsilon}\right)_{12} \rightarrow E_{12}$ and $\left(E^{\varepsilon} y_{\varepsilon}\right)_{22} \rightarrow E_{22}$ in $L^{2}(S)$. We let $\tilde{\gamma}: I \rightarrow \mathbb{R}$ and $z: S \rightarrow \mathbb{R}$ be functions such that

$$
\begin{aligned}
Q_{W}^{2}\left(\tilde{w}^{\prime \prime}, \tilde{\theta}^{\prime}, \tilde{\gamma}\right) & =Q_{W}^{1}\left(\tilde{w}^{\prime \prime}, \tilde{\theta}^{\prime}\right) \quad \text { and } \\
Q_{W}^{0}\left(\partial_{1} \tilde{y}_{1}+\frac{1}{2} \tilde{w}^{\prime \prime 2}\right) & =Q_{W}^{2}\left(\partial_{1} \tilde{y}_{1}+\frac{1}{2} \tilde{w}^{\prime \prime 2}, 0, z\right) .
\end{aligned}
$$

As $Q_{W}^{2}$ is positive definite on $\mathbb{R}_{\mathrm{sym}}^{2 \times 2}$ and $(\tilde{y}, \tilde{w}, \tilde{\theta}) \in \mathscr{S}^{1 D}$, we find that $\tilde{\gamma} \in L^{2}(I)$ and $z \in L^{2}(S)$. By standard density arguments for $L^{2}$ - and $W^{1,2}$-spaces, there exist functions $\theta_{\varepsilon} \in C_{c}^{\infty}(I)$ and $\tilde{\gamma}_{\varepsilon}, \zeta_{\varepsilon} \in C_{c}^{\infty}(S)$ such that $\theta_{\varepsilon} \rightarrow \tilde{\theta}-\theta$ in $W^{1,2}(I), \varepsilon \theta_{\varepsilon}^{\prime}, \varepsilon \theta_{\varepsilon}^{\prime \prime} \rightarrow 0$ in $L^{2}(I), \tilde{\gamma}_{\varepsilon} \rightarrow \tilde{\gamma}-\gamma, \varepsilon \partial_{1} \tilde{\gamma}_{\varepsilon} \rightarrow 0, \varepsilon^{2} \partial_{11}^{2} \tilde{\gamma}_{\varepsilon} \rightarrow 0$ in $L^{2}(S)$, and $\zeta_{\varepsilon} \rightarrow z-E_{22}, \varepsilon \partial_{1} \zeta_{\varepsilon} \rightarrow 0$ in $L^{2}(S)$. We define the recovery sequence for the vertical displacement $\tilde{w}_{\varepsilon}$ as

$$
\tilde{w}_{\varepsilon}(x)=w_{\varepsilon}(x)+\tilde{w}\left(x_{1}\right)-w\left(x_{1}\right)+\varepsilon x_{2} \theta_{\varepsilon}\left(x_{1}\right)+\varepsilon^{2} \int_{-1 / 2}^{x_{2}} \int_{-1 / 2}^{t} \tilde{\gamma}_{\varepsilon}\left(x_{1}, s\right) \mathrm{d} s \mathrm{~d} t .
$$

By recalling (2.7), we compute

$$
\nabla_{\varepsilon} w_{\varepsilon}(x)=\left(\begin{array}{c}
\partial_{1} w_{\varepsilon}(x)+\tilde{w}^{\prime}\left(x_{1}\right)-w^{\prime}\left(x_{1}\right)+\varepsilon x_{2} \theta_{\varepsilon}^{\prime}\left(x_{1}\right)+\varepsilon^{2} \int_{-1 / 2}^{x_{2}} \int_{-1 / 2}^{t} \partial_{1} \tilde{\gamma}_{\varepsilon}\left(x_{1}, s\right) \mathrm{d} s \mathrm{~d} t \\
\frac{1}{\varepsilon} \partial_{2} w_{\varepsilon}(x)+\theta_{\varepsilon}\left(x_{1}\right)+\varepsilon \int_{-1 / 2}^{x_{2}} \tilde{\gamma}_{\varepsilon}\left(x_{1}, s\right) \mathrm{d} s
\end{array}\right)
$$

and further see that the entries of the scaled Hessian $\nabla_{\varepsilon}^{2} \tilde{w}_{\varepsilon}(x)$ are given by

$$
\begin{aligned}
\partial_{11}^{2} w_{\varepsilon}(x)= & \partial_{11} w_{\varepsilon}(x)+\tilde{w}^{\prime \prime}\left(x_{1}\right)-w^{\prime \prime}\left(x_{1}\right)+\varepsilon x_{2} \theta_{\varepsilon}^{\prime \prime}\left(x_{1}\right) \\
& +\varepsilon^{2} \int_{-1 / 2}^{x_{2}} \int_{-1 / 2}^{t} \partial_{11}^{2} \tilde{\gamma}_{\varepsilon}\left(x_{1}, s\right) \mathrm{d} s \mathrm{~d} t \\
\frac{1}{\varepsilon} \partial_{12}^{2} w_{\varepsilon}(x)= & \frac{1}{\varepsilon} \partial_{12} w_{\varepsilon}(x)+\theta_{\varepsilon}^{\prime}\left(x_{1}\right)+\varepsilon \int_{-1 / 2}^{x_{2}} \partial_{1} \tilde{\gamma}_{\varepsilon}\left(x_{1}, s\right) \mathrm{d} s \\
\frac{1}{\varepsilon^{2}} \partial_{22}^{2} w_{\varepsilon}(x)= & \frac{1}{\varepsilon^{2}} \partial_{22} w_{\varepsilon}(x)+\tilde{\gamma}_{\varepsilon}\left(x_{1}, x_{2}\right)
\end{aligned}
$$

Let us now address the in-plane displacements. We define

$$
\begin{aligned}
& \left(\bar{y}_{\varepsilon}\right)_{1}\left(x_{1}, x_{2}\right):=\tilde{y}_{1}(x)-y_{1}(x)+\varepsilon x_{2}\left(w^{\prime}\left(x_{1}\right) \theta\left(x_{1}\right)-\tilde{w}^{\prime}\left(x_{1}\right) \tilde{\theta}\left(x_{1}\right)\right), \\
& \left(\bar{y}_{\varepsilon}\right)_{2}\left(x_{1}, x_{2}\right):=\tilde{y}_{2}(x)-y_{2}(x)-\frac{\varepsilon^{2}}{2} x_{2} \tilde{\theta}^{2}\left(x_{1}\right)+\varepsilon^{2} \int_{-1 / 2}^{x_{2}} \zeta_{\varepsilon}\left(x_{1}, s\right) \mathrm{d} s .
\end{aligned}
$$


The characterization in (2.9) implies that $\partial_{2} y_{1}=-\partial_{1} y_{2}, \partial_{2} \tilde{y}_{1}=-\partial_{1} \tilde{y}_{2}$, and $\partial_{2} y_{2}=\partial_{2} \tilde{y}_{2}=0$. Then, by the definition of $E^{\varepsilon}$ we have

$$
\begin{aligned}
\left(E^{\varepsilon} \bar{y}_{\varepsilon}\right)_{11}= & \partial_{1} \tilde{y}_{1}(x)-\partial_{1} y_{1}(x)+\varepsilon x_{2}\left(w^{\prime}\left(x_{1}\right) \theta^{\prime}\left(x_{1}\right)+w^{\prime \prime}\left(x_{1}\right) \theta\left(x_{1}\right)\right. \\
& \left.-\tilde{w}^{\prime}\left(x_{1}\right) \tilde{\theta}^{\prime}\left(x_{1}\right)-\tilde{w}^{\prime \prime}\left(x_{1}\right) \tilde{\theta}\left(x_{1}\right)\right) \\
\left(E^{\varepsilon} \bar{y}_{\varepsilon}\right)_{12}= & \frac{1}{2}\left(w^{\prime}\left(x_{1}\right) \theta\left(x_{1}\right)-\tilde{w}^{\prime}\left(x_{1}\right) \tilde{\theta}\left(x_{1}\right)\right) \\
& -\frac{\varepsilon x_{2}}{2} \tilde{\theta}\left(x_{1}\right) \tilde{\theta}^{\prime}\left(x_{1}\right)+\frac{\varepsilon}{2} \int_{-1 / 2}^{x_{2}} \partial_{1} \zeta_{\varepsilon}\left(x_{1}, s\right) \mathrm{d} s \\
\left(E^{\varepsilon} \bar{y}_{\varepsilon}\right)_{22}= & -\frac{1}{2} \tilde{\theta}^{2}\left(x_{1}\right)+\zeta_{\varepsilon}\left(x_{1}, x_{2}\right) .
\end{aligned}
$$

The construction ensures that $\bar{y}_{\varepsilon} \in W^{1,2}\left(S ; \mathbb{R}^{2}\right)$. We let $\tilde{y}_{\varepsilon}:=y_{\varepsilon}+\bar{y}_{\varepsilon}$. Eventually, we define $\tilde{u}_{\varepsilon}$ and $\tilde{v}_{\varepsilon}$ such that $\left(\tilde{y}_{\varepsilon}\right)_{1}\left(x_{1}, x_{2}\right)=\left(\tilde{u}_{\varepsilon}\right)_{1}\left(x_{1}, \varepsilon x_{2}\right),\left(\tilde{y}_{\varepsilon}\right)_{2}\left(x_{1}, x_{2}\right)=$ $\varepsilon\left(\tilde{u}_{\varepsilon}\right)_{2}\left(x_{1}, \varepsilon x_{2}\right)$, and $\tilde{w}_{\varepsilon}\left(x_{1}, x_{2}\right)=\tilde{v}_{\varepsilon}\left(x_{1}, \varepsilon x_{2}\right)$, see $(2.6)$. As $\theta, \tilde{\theta}, \theta_{\varepsilon}, \tilde{y}-y$, and $\tilde{w}-w$ vanish on $\partial I$, and $\gamma_{\varepsilon}, \zeta_{\varepsilon}$ vanish on $\partial S$, an inspection of (5.7)-(5.8) shows that $\left(\tilde{u}_{\varepsilon}, \tilde{v}_{\varepsilon}\right) \in \mathscr{S}_{\varepsilon}^{2 D}$.

Step 2: Proof of (5.4). Due to Proposition 2.1 and the compact embedding $W^{1,2}(S) \subset \subset L^{4}(S)$, we find that $\nabla_{\varepsilon} w_{\varepsilon} \rightarrow\left(w^{\prime}, \theta\right)$ in $L^{4}\left(S ; \mathbb{R}^{2}\right)$ and hence

$$
\nabla_{\varepsilon} w_{\varepsilon} \otimes \nabla_{\varepsilon} w_{\varepsilon} \rightarrow\left(w^{\prime}, \theta\right) \otimes\left(w^{\prime}, \theta\right) \quad \text { in } \quad L^{2}\left(S ; \mathbb{R}^{2 \times 2}\right) .
$$

Similarly, due to the fact that $\partial_{1} w_{\varepsilon} \rightarrow w^{\prime}$ in $L^{4}(S), \frac{1}{\varepsilon} \partial_{2} w_{\varepsilon} \rightarrow \theta$ in $L^{4}(S)$, and $\theta_{\varepsilon} \rightarrow \tilde{\theta}-\theta$ in $W^{1,2}(I)$, we have that $\nabla_{\varepsilon} \tilde{w}_{\varepsilon} \rightarrow\left(\tilde{w}^{\prime}, \tilde{\theta}\right)$ in $L^{4}\left(S ; \mathbb{R}^{2}\right)$, and thus

$$
\nabla_{\varepsilon} \tilde{w}_{\varepsilon} \otimes \nabla_{\varepsilon} \tilde{w}_{\varepsilon} \rightarrow\left(\tilde{w}^{\prime}, \tilde{\theta}\right) \otimes\left(\tilde{w}^{\prime}, \tilde{\theta}\right) \quad \text { in } \quad L^{2}\left(S ; \mathbb{R}^{2 \times 2}\right) .
$$

This along with $\zeta_{\varepsilon} \rightarrow z-E_{22}$ in $L^{2}(S)$ and $\tilde{y}_{\varepsilon}-y_{\varepsilon}=\bar{y}_{\varepsilon}$ implies by an elementary computation

$$
\begin{aligned}
& E^{\varepsilon}\left(\tilde{y}_{\varepsilon}-y_{\varepsilon}\right)+\frac{1}{2} \nabla_{\varepsilon} \tilde{w}_{\varepsilon} \otimes \nabla_{\varepsilon} \tilde{w}_{\varepsilon}-\frac{1}{2} \nabla_{\varepsilon} w_{\varepsilon} \otimes \nabla_{\varepsilon} w_{\varepsilon} \\
& \longrightarrow\left(\begin{array}{rr}
\partial_{1} \tilde{y}_{1}-\partial_{1} y_{1}+\frac{1}{2}\left(\tilde{w}^{\prime 2}-w^{\prime 2}\right) & 0 \\
0 & z-E_{22}-\frac{1}{2} \theta^{2}
\end{array}\right)
\end{aligned}
$$

strongly in $L^{2}\left(S ; \mathbb{R}^{2 \times 2}\right)$. Moreover, by $\theta_{\varepsilon} \rightarrow \tilde{\theta}-\theta$ in $W^{1,2}(I)$ and $\tilde{\gamma}_{\varepsilon} \rightarrow \tilde{\gamma}-\gamma$ in $L^{2}(S)$, we have

$$
\nabla_{\varepsilon}^{2}\left(\tilde{w}_{\varepsilon}-w_{\varepsilon}\right) \rightarrow\left(\begin{array}{cc}
\tilde{w}^{\prime \prime}-w^{\prime \prime} & \tilde{\theta}^{\prime}-\theta^{\prime} \\
\tilde{\theta}^{\prime}-\theta^{\prime} & \tilde{\gamma}-\gamma
\end{array}\right)
$$

strongly in $L^{2}\left(S ; \mathbb{R}^{2 \times 2}\right)$. Proposition 2.1 and (5.9) also yield

$$
E^{\varepsilon} y_{\varepsilon}+\frac{1}{2} \nabla_{\varepsilon} w_{\varepsilon} \otimes \nabla_{\varepsilon} w_{\varepsilon} \rightarrow\left(\begin{array}{cc}
\partial_{1} y_{1}+\frac{1}{2} w^{\prime 2} & E_{12}+w^{\prime} \theta \\
E_{12}+w^{\prime} \theta & E_{22}+\frac{1}{2} \theta^{2}
\end{array}\right) \quad \text { and } \quad \nabla_{\varepsilon}^{2} w_{\varepsilon} \rightarrow\left(\begin{array}{cc}
w^{\prime \prime} & \theta^{\prime} \\
\theta^{\prime} & \gamma
\end{array}\right)
$$

weakly in $L^{2}\left(S ; \mathbb{R}^{2 \times 2}\right)$. Then, by (5.10), (5.11), and (H2) (including explicitly the $\varepsilon$-dependence of $\left.Q_{R, \varepsilon}^{2}\right)$ we find that 


$$
\begin{aligned}
& \int_{S} Q_{R, \varepsilon}^{2}\left(E^{\varepsilon}\left(\tilde{y}_{\varepsilon}-y_{\varepsilon}\right)+\frac{1}{2} \nabla_{\varepsilon} \tilde{w}_{\varepsilon} \otimes \nabla_{\varepsilon} \tilde{w}_{\varepsilon}-\frac{1}{2} \nabla_{\varepsilon} w_{\varepsilon} \otimes \nabla_{\varepsilon} w_{\varepsilon}\right) \\
& \quad+\frac{1}{12} \int_{S} Q_{R, \varepsilon}^{2}\left(\nabla_{\varepsilon}^{2}\left(\tilde{w}_{\varepsilon}-w_{\varepsilon}\right)\right) \\
& \quad \longrightarrow \int_{S} Q_{R}^{0}\left(\partial_{1} \tilde{y}_{1}-\partial_{1} y_{1}+\frac{1}{2}\left(\tilde{w}^{\prime 2}-w^{\prime 2}\right)\right)+\frac{1}{12} \int_{I} Q_{R}^{1}\left(\tilde{w}^{\prime \prime}-w^{\prime \prime}, \tilde{\theta}^{\prime}-\theta^{\prime}\right) .
\end{aligned}
$$

This shows (5.4).

Step 3: Proof of (5.3). We additionally use (5.5) and the fact that a product of sequences converges weakly in $L^{1}$ if one factor converges weakly and the other one strongly: by (5.11), (5.12) we obtain

$$
\begin{aligned}
\int_{S} & Q_{W}^{2}\left(\nabla_{\varepsilon}^{2} w_{\varepsilon}\right)-\int_{S} Q_{W}^{2}\left(\nabla_{\varepsilon}^{2} \tilde{w}_{\varepsilon}\right) \\
& =\int_{S} Q_{W}^{2}\left(\nabla_{\varepsilon}^{2} w_{\varepsilon}-\nabla_{\varepsilon}^{2} \tilde{w}_{\varepsilon}\right)+2 \mathbb{C}_{W}^{2}\left[\nabla_{\varepsilon}^{2} w_{\varepsilon}-\nabla_{\varepsilon}^{2} \tilde{w}_{\varepsilon}, \nabla_{\varepsilon}^{2} \tilde{w}_{\varepsilon}\right] \\
& \longrightarrow \int_{S} Q_{W}^{2}\left(\left(\begin{array}{cc}
w^{\prime \prime}-\tilde{w}^{\prime \prime} & \theta^{\prime}-\tilde{\theta}^{\prime} \\
\theta^{\prime}-\tilde{\theta}^{\prime} & \gamma-\tilde{\gamma}
\end{array}\right)\right)+2 \mathbb{C}_{W}^{2}\left[\left(\begin{array}{cc}
w^{\prime \prime}-\tilde{w}^{\prime \prime} & \theta^{\prime}-\tilde{\theta}^{\prime} \\
\theta^{\prime}-\tilde{\theta}^{\prime} & \gamma-\tilde{\gamma}
\end{array}\right),\left(\begin{array}{cc}
\tilde{w}^{\prime \prime} & \tilde{\theta}^{\prime} \\
\tilde{\theta}^{\prime} & \tilde{\gamma}
\end{array}\right)\right] \\
& =L_{1}:=\int_{S} Q_{W}^{2}\left(\left(\begin{array}{cc}
w^{\prime \prime} & \theta^{\prime} \\
\theta^{\prime} & \gamma
\end{array}\right)\right)-\int_{S} Q_{W}^{2}\left(\left(\begin{array}{cc}
\tilde{w}^{\prime \prime} & \tilde{\theta}^{\prime} \\
\tilde{\theta}^{\prime} & \tilde{\gamma}
\end{array}\right)\right) .
\end{aligned}
$$

By (2.10) and (5.6) this implies

$$
L_{1} \geq \int_{I} Q_{W}^{1}\left(\left(w^{\prime \prime}, \theta^{\prime}\right)\right)-\int_{I} Q_{W}^{1}\left(\left(\tilde{w}^{\prime \prime}, \tilde{\theta}^{\prime}\right)\right) .
$$

Similarly, due to (5.5), (5.10), and (5.12), we have

$$
\begin{aligned}
\int_{S} Q_{W}^{2}\left(E^{\varepsilon} y_{\varepsilon}\right. & \left.+\frac{1}{2} \nabla_{\varepsilon} w_{\varepsilon} \otimes \nabla_{\varepsilon} w_{\varepsilon}\right)-\int_{S} Q_{W}^{2}\left(E^{\varepsilon} \tilde{y}_{\varepsilon}+\nabla_{\varepsilon} \tilde{w}_{\varepsilon} \otimes \nabla_{\varepsilon} \tilde{w}_{\varepsilon}\right) \\
\longrightarrow L_{2}:= & \int_{S} Q_{W}^{2}\left(\left(\begin{array}{cc}
\partial_{1} y_{1}+\frac{1}{2} w^{\prime 2} & E_{12}+w^{\prime} \theta \\
E_{12}+w^{\prime} \theta & E_{22}+\frac{1}{2} \theta^{2}
\end{array}\right)\right) \\
& -\int_{S} Q_{W}^{2}\left(\left(\begin{array}{cc}
\partial_{1} \tilde{y}_{1}+\frac{1}{2} \tilde{w}^{\prime 2} & E_{12}+w^{\prime} \theta \\
E_{12}+w^{\prime} \theta & z
\end{array}\right)\right) .
\end{aligned}
$$

By (H2) we have $\arg \min _{q_{12}} Q_{W}^{2}\left(q_{11}, q_{12}, q_{22}\right)=0$ and thus $Q_{W}^{2}\left(q_{11}, q_{12}, q_{22}\right)=$ $Q_{W}^{2}\left(q_{11}, 0, q_{22}\right)+b q_{12}^{2}$ for some $b>0$. This along with (2.10) and (5.6) shows

$$
L_{2} \geq \int_{S} Q_{W}^{0}\left(\partial_{1} y_{1}+\frac{1}{2}\left|w^{\prime}\right|^{2}\right)-\int_{S} Q_{W}^{0}\left(\partial_{1} \tilde{y}_{1}+\frac{1}{2}\left|\tilde{w}^{\prime}\right|^{2}\right)
$$

which in combination with (5.14) concludes the proof of (5.3).

Step 4: Adaptions for $(\mathbf{H 1})$. We now suppose that $(\mathbf{H 1})$ holds. We redefine $\tilde{\gamma}$ and $z$ differently compared to (5.6): let $\tilde{\gamma}=\gamma$ and $z=\theta^{2} / 2+E_{22}$. Then, (5.10)-(5.11) imply

$$
\begin{aligned}
\int_{S} Q_{R}^{2}\left(E^{\varepsilon}\left(\tilde{y}_{\varepsilon}-y_{\varepsilon}\right)+\frac{1}{2} \nabla_{\varepsilon} \tilde{w}_{\varepsilon} \otimes \nabla_{\varepsilon} \tilde{w}_{\varepsilon}-\frac{1}{2} \nabla_{\varepsilon} w_{\varepsilon} \otimes \nabla_{\varepsilon} w_{\varepsilon}\right) \\
+\frac{1}{12} \int_{S} Q_{R}^{2}\left(\nabla_{\varepsilon}^{2}\left(\tilde{w}_{\varepsilon}-w_{\varepsilon}\right)\right)
\end{aligned}
$$




$$
\begin{aligned}
& \longrightarrow \int_{S} Q_{R}^{2}\left(\left(\begin{array}{cc}
\partial_{1} \tilde{y}_{1}-\partial_{1} y_{1}+\frac{1}{2}\left(\tilde{w}^{\prime 2}-w^{\prime 2}\right) & 0 \\
0 & 0
\end{array}\right)\right) \\
& +\frac{1}{12} \int_{I} Q_{R}^{2}\left(\left(\begin{array}{cc}
\tilde{w}^{\prime \prime}-w^{\prime \prime} & \tilde{\theta}^{\prime}-\theta^{\prime} \\
\tilde{\theta}^{\prime}-\theta^{\prime} & 0
\end{array}\right)\right) .
\end{aligned}
$$

This along with (H1) shows (5.4). By (5.13) and (5.15) we get $\phi_{\varepsilon}\left(u_{\varepsilon}, v_{\varepsilon}\right)-$ $\phi_{\varepsilon}\left(\tilde{u}_{\varepsilon}, \tilde{v}_{\varepsilon}\right) \rightarrow \frac{1}{2} L_{2}+\frac{1}{24} L_{1}$, where $L_{1}$ and $L_{2}$ are given for $z=\theta^{2} / 2+E_{22}$ and $\tilde{\gamma}=\gamma$, respectively. The condition in $(\mathbf{H 1})$ implies that $Q_{W}^{2}\left(q_{11}, q_{12}, q_{22}\right)=$ $a q_{11}^{2}+b q_{12}^{2}+c q_{22}^{2}$ for suitable $a, b, c>0$. In view of (2.16), this yields $\frac{1}{2} L_{2}+$ $\frac{1}{24} L_{1}=\phi_{0}(y, w, \theta)-\phi_{0}(\tilde{y}, \tilde{w}, \tilde{\theta})$ and concludes the proof of $(5.3)$.

Remark 5.5. (Recovery sequences) The ansatz for the recovery sequence in (5.7) and (5.8) is similar to [20] with the difference that (a) (5.8) is slightly modified to deal with boundary conditions and (b) we add suitable corrections concerning the difference of $(y, w, \theta)$ and $(\tilde{y}, \tilde{w}, \tilde{\theta})$ and the variables $\gamma$ and $E_{22}$ resulting from Proposition 2.1.

(a) The definition ensures that we can construct recovery sequences for $(\tilde{y}, \tilde{w}, \tilde{\theta})$ in Theorem 5.1(ii): choose $\left(u_{\varepsilon}, v_{\varepsilon}\right)=(0,0)$ and $(y, w, \theta)=(0,0,0)$ and construct $\left(\tilde{u}_{\varepsilon}, \tilde{v}_{\varepsilon}\right) \in \mathscr{S}_{\varepsilon}^{2 D}$ as in the above proof. Then we can again obtain (5.3). This along with $\phi_{\varepsilon}\left(u_{\varepsilon}, v_{\varepsilon}\right)=0=\phi_{0}(y, w, \theta)$ yields $\limsup _{\varepsilon \rightarrow 0} \phi_{\varepsilon}\left(\tilde{u}_{\varepsilon}, \tilde{v}_{\varepsilon}\right) \leq \phi_{0}(\tilde{y}, \tilde{w}, \tilde{\theta})$.

(b) The correction ensures strong convergence in (5.10)-(5.11) which allows to pass to the limit in the metric term. Clearly, this is not relevant in the purely static setting [20]. Our construction does not only take the limiting configuration $(y, w, \theta)$ into account, but also the limits $\gamma$ and $E_{22}$ provided by Proposition 2.1. Therefore, as $\gamma$ and $E_{22}$ may be $x_{2}$-dependent, in contrast to [20], we need to construct suitable approximations in $C_{c}^{\infty}(S)$ instead of using correction terms only defined on the interval $I$. In the case $(\mathbf{H} 2)$, being more general for $Q_{W}^{2}$, the quantities $z-E_{22}-\frac{1}{2} \theta^{2}$ and $\tilde{\gamma}-\gamma$ do not vanish in general and therefore an additional assumption on $Q_{R, \varepsilon}^{2}$ is required. In particular, due to the involved relaxation, see (5.14) and (5.16), we only expect an inequality in (5.3). The argument for (H1) is simpler and the mutual recovery sequence even satisfies an equality in (5.3) which can be deduced from the structure of the quadratic form.

Now we derive the lower semicontinuity of the slopes. The same argument for a single energy/metric was used in the proof of [23, Theorem 3.2]. We include the proof here for the reader's convenience.

Theorem 5.6. (Lower semicontinuity of slopes) Suppose that (H1) or (H2) holds. Then, for each sequence $\left(u_{\varepsilon}, v_{\varepsilon}\right)_{\varepsilon}$ with $\left(u_{\varepsilon}, v_{\varepsilon}\right) \in \mathscr{S}_{\varepsilon, M}^{2 D}$ such that $\left(u_{\varepsilon}, v_{\varepsilon}\right) \stackrel{\pi \sigma}{\longrightarrow}(y, w, \theta)$ we have

$$
\liminf _{\varepsilon \rightarrow 0}\left|\partial \phi_{\varepsilon}\right|_{\mathcal{D}_{\varepsilon}}\left(u_{\varepsilon}, v_{\varepsilon}\right) \geq\left|\partial \phi_{0}\right|_{\mathcal{D}_{0}}(y, w, \theta) .
$$


Proof. First, by Theorem 5.1(i) we get $\phi_{0}(y, w, \theta) \leq M$. Let $\delta>0$ and use Lemma $4.3(\mathrm{i})$ to find $(\tilde{y}, \tilde{w}, \tilde{\theta}) \in \mathscr{S}^{1 D}$ with $(\tilde{y}, \tilde{w}, \tilde{\theta}) \neq(y, w, \theta)$ such that

$\left|\partial \phi_{0}\right|_{\mathcal{D}_{0}}(y, w, \theta)-\delta \leq \frac{\left(\phi_{0}(y, w, \theta)-\phi_{0}(\tilde{y}, \tilde{w}, \tilde{\theta})-\Phi_{M}^{2}\left(\mathcal{D}_{0}((y, w, \theta),(\tilde{y}, \tilde{w}, \tilde{\theta}))\right)\right)^{+}}{\Phi^{1}\left(\mathcal{D}_{0}((y, w, \theta),(\tilde{y}, \tilde{w}, \tilde{\theta}))\right)}$.

Let $\left(\tilde{u}_{\varepsilon}, \tilde{v}_{\varepsilon}\right)_{\varepsilon}$ be the mutual recovery sequence constructed in Lemma 5.3. Then, Lemma 5.3 yields

$$
\begin{aligned}
& \left|\partial \phi_{0}\right|_{\mathcal{D}_{0}}(y, w, \theta)-\delta \\
& \quad \leq \liminf _{\varepsilon \rightarrow 0} \frac{\left(\phi_{\varepsilon}\left(u_{\varepsilon}, v_{\varepsilon}\right)-\phi_{\varepsilon}\left(\tilde{u}_{\varepsilon}, \tilde{v}_{\varepsilon}\right)-\Phi_{M}^{2}\left(\mathcal{D}_{\varepsilon}\left(\left(u_{\varepsilon}, v_{\varepsilon}\right),\left(\tilde{u}_{\varepsilon}, \tilde{v}_{\varepsilon}\right)\right)\right)\right)^{+}}{\Phi^{1}\left(\mathcal{D}_{\varepsilon}\left(\left(u_{\varepsilon}, v_{\varepsilon}\right),\left(\tilde{u}_{\varepsilon}, \tilde{v}_{\varepsilon}\right)\right)\right)} .
\end{aligned}
$$

In view of Lemma 4.1(ii), taking the supremum and sending $\delta \rightarrow 0$ yields

$$
\liminf _{\varepsilon \rightarrow 0}\left|\partial \phi_{\varepsilon}\right|_{\mathcal{D}_{\varepsilon}}\left(u_{\varepsilon}, v_{\varepsilon}\right) \geq\left|\partial \phi_{0}\right|_{\mathcal{D}}(y, w, \theta)
$$

and concludes the proof.

\subsection{Convergence of minimizers and strong convergence}

In the next theorem, we analyze time-discrete solutions introduced in (3.1). We denote by $\boldsymbol{\Phi}_{\varepsilon}$ the two-dimensional and by $\boldsymbol{\Phi}_{0}$ the one-dimensional scheme. We show that minimizers of the two-dimensional discretization scheme converge to minimizers of the one-dimensional scheme.

Theorem 5.7. (Convergence of minimizer of the schemes) Suppose that (H1) or (H2) holds. Let $\left(u_{\varepsilon}, v_{\varepsilon}\right)_{\varepsilon}$ with $\left(u_{\varepsilon}, v_{\varepsilon}\right) \in \mathscr{S}_{\varepsilon}^{2 D}$ be a sequence such that

$$
\left(u_{\varepsilon}, v_{\varepsilon}\right) \stackrel{\pi \sigma}{\rightarrow}(y, w, \theta) \quad \text { and } \quad \phi_{\varepsilon}\left(u_{\varepsilon}, v_{\varepsilon}\right) \rightarrow \phi_{0}(y, w, \theta)
$$

as $\varepsilon \rightarrow 0$. Moreover, let $\tau>0$ and consider a sequence $\left(Y_{\varepsilon, \tau}\right)_{\varepsilon}, Y_{\varepsilon, \tau} \in \mathscr{S}_{\varepsilon}^{2 D}$, such that

$$
Y_{\varepsilon, \tau} \arg \min _{z \in \mathscr{S}_{\varepsilon}^{2 D}} \boldsymbol{\Phi}_{\varepsilon}\left(\tau,\left(u_{\varepsilon}, v_{\varepsilon}\right), z\right) \text { for all } \varepsilon>0 \quad \text { and } \quad Y_{\varepsilon, \tau} \stackrel{\pi \sigma}{\rightarrow} U_{\tau} \quad \text { as } \varepsilon \rightarrow 0 .
$$

Then,

(i) $\quad U_{\tau}=\arg \min _{v \in \mathscr{S}^{1 D}} \boldsymbol{\Phi}_{0}(\tau,(y, w, \theta), v)$,

(ii) $\boldsymbol{\Phi}_{\varepsilon}\left(\tau,\left(u_{\varepsilon}, v_{\varepsilon}\right), Y_{\varepsilon, \tau}\right) \rightarrow \boldsymbol{\Phi}_{0}\left(\tau,(y, w, \theta), U_{\tau}\right)$,

(iii) $\phi_{\varepsilon}\left(Y_{\varepsilon, \tau}\right) \rightarrow \phi_{0}\left(U_{\tau}\right)$.

Proof. The proof is based on the fundamental property that $\Gamma$-convergence induces convergence of minima and minimizers. By Theorem 5.1(i) and Theorem 5.2 we have

$$
\inf _{v \in \mathscr{S}^{1 D}} \boldsymbol{\Phi}_{0}(\tau,(y, w, \theta), v) \leq \boldsymbol{\Phi}_{0}\left(\tau,(y, w, \theta), U_{\tau}\right) \leq \liminf _{\varepsilon \rightarrow 0} \boldsymbol{\Phi}_{\varepsilon}\left(\tau,\left(u_{\varepsilon}, v_{\varepsilon}\right), Y_{\varepsilon, \tau}\right) .
$$

Let $\delta>0$ and consider $(\tilde{y}, \tilde{w}, \tilde{\theta}) \in \mathscr{S}^{1 D}$ such that

$$
\inf _{v \in \mathscr{S}^{1 D}} \boldsymbol{\Phi}_{0}(\tau,(y, w, \theta), v)+\delta \geq \mathbf{\Phi}_{0}(\tau,(y, w, \theta),(\tilde{y}, \tilde{w}, \tilde{\theta})) .
$$


In view of Lemma 5.3 and $(5.17)$, we find a mutual recovery sequence $\left(\tilde{u}_{\varepsilon}, \tilde{v}_{\varepsilon}\right)_{\varepsilon}$ such that

$$
\begin{aligned}
\inf _{v \in \mathscr{S}^{1 D}} \boldsymbol{\Phi}_{0}(\tau,(y, w, \theta), v)+\delta \geq & \limsup _{\varepsilon \rightarrow 0} \phi_{\varepsilon}\left(\tilde{u}_{\varepsilon}, \tilde{v}_{\varepsilon}\right) \\
& +\limsup _{\varepsilon \rightarrow 0} \frac{1}{2 \tau} \mathcal{D}_{\varepsilon}^{2}\left(\left(u_{\varepsilon}, v_{\varepsilon}\right),\left(\tilde{u}_{\varepsilon}, \tilde{v}_{\varepsilon}\right)\right) \\
\geq & \limsup _{\varepsilon \rightarrow 0} \Phi_{\varepsilon}\left(\tau,\left(u_{\varepsilon}, v_{\varepsilon}\right),\left(\tilde{u}_{\varepsilon}, \tilde{v}_{\varepsilon}\right)\right) \\
\geq & \limsup _{\varepsilon \rightarrow 0} \boldsymbol{\Phi}_{\varepsilon}\left(\tau,\left(u_{\varepsilon}, v_{\varepsilon}\right), Y_{\varepsilon, \tau}\right),
\end{aligned}
$$

where the last step follows from minimality of $Y_{\varepsilon, \tau}$. By sending $\delta \rightarrow 0$, (5.18) and (5.19) imply (i) and (ii) as all inequalities are actually equalities. Finally, (iii) follows by (ii) and Theorems 5.1 (i) and 5.2.

The following lemma helps us to show that the convergence of the sequences in Theorems 2.2 and 2.3 holds in a strong sense.

Lemma 5.8. (Strong convergence of recovery sequences) Let $\left(u_{\varepsilon}, v_{\varepsilon}\right)_{\varepsilon}$ with $\left(u_{\varepsilon}, v_{\varepsilon}\right) \in \mathscr{S}_{\varepsilon}^{2 D}$ be a sequence such that

$$
\left(u_{\varepsilon}, v_{\varepsilon}\right) \stackrel{\pi \sigma}{\longrightarrow}(y, w, \theta) \quad \text { and } \quad \phi_{\varepsilon}\left(u_{\varepsilon}, v_{\varepsilon}\right) \rightarrow \phi_{0}(y, w, \theta) .
$$

Then, we have $\left(u_{\varepsilon}, v_{\varepsilon}\right) \stackrel{\pi \rho}{\rightarrow}(y, w, \theta)$.

Proof. Let $\left(y_{\varepsilon}, w_{\varepsilon}\right)$ be the scaled version corresponding to $\left(u_{\varepsilon}, v_{\varepsilon}\right)$ introduced in (2.6). By Proposition 2.1 we have

$$
\nabla_{\varepsilon}^{2} w_{\varepsilon} \rightarrow\left(\begin{array}{cc}
w^{\prime \prime} & \theta^{\prime} \\
\theta^{\prime} & \gamma
\end{array}\right) \quad \text { and } \quad E^{\varepsilon} y_{\varepsilon}+\frac{1}{2} \nabla_{\varepsilon} w_{\varepsilon} \otimes \nabla_{\varepsilon} w_{\varepsilon} \rightarrow\left(\begin{array}{cc}
\partial_{1} y_{1}+\frac{1}{2} w^{\prime \prime 2} & E_{12}+\frac{1}{2} w^{\prime} \theta \\
E_{12}+\frac{1}{2} w^{\prime} \theta & E_{22}+\frac{1}{2} \theta^{2}
\end{array}\right)
$$

weakly in $L^{2}\left(S ; \mathbb{R}_{\text {sym }}^{2 \times 2}\right)$, for suitable functions $\gamma \in L^{2}(S), E_{12} \in L^{2}(S)$, and $E_{22} \in L^{2}(S)$. It suffices to show that these convergences are strong. Then all (strong) convergences indicated in Proposition 2.1 follow where $E^{\varepsilon} y_{\varepsilon}$ converges due to the compact embedding $W^{1,2}(S) \subset \subset L^{4}(S)$. We start the proof by observing that the convexity of $Q_{W}^{2}$ and $\left(u_{\varepsilon}, v_{\varepsilon}\right) \stackrel{\pi \sigma}{\rightarrow}(y, w, \theta)$ yield

$$
\begin{gathered}
\liminf _{\varepsilon \rightarrow 0} \phi_{\varepsilon}\left(u_{\varepsilon}, v_{\varepsilon}\right) \geq \frac{1}{2} \int_{S} Q_{W}^{2}\left(\left(\begin{array}{cc}
\partial_{1} y_{1}+\frac{1}{2} w^{\prime 2} & E_{12}+\frac{1}{2} w^{\prime} \theta \\
E_{12}+\frac{1}{2} w^{\prime} \theta & E_{22}+\frac{1}{2} \theta^{2}
\end{array}\right)\right) \\
+\frac{1}{24} \int_{S} Q_{W}^{2}\left(\left(\begin{array}{cc}
w^{\prime \prime} & \theta^{\prime} \\
\theta^{\prime} & \gamma
\end{array}\right)\right),
\end{gathered}
$$

where we used the representation of $\phi_{\varepsilon}$ in (5.1). By (2.10)-(2.11) and (2.16), the right-hand side is bigger or equal than $\phi_{0}(y, w, \theta)$. This along with $\lim _{\varepsilon \rightarrow 0}$ $\phi_{\varepsilon}\left(u_{\varepsilon}, v_{\varepsilon}\right)=\phi_{0}(y, w, \theta)$ shows that all inequalities are actually equalities. Using once more (5.20) and the convexity of $Q_{W}^{2}$, we derive by (5.1) that

$$
\begin{aligned}
& \frac{1}{2} \int_{S} Q_{W}^{2}\left(E^{\varepsilon} y_{\varepsilon}+\frac{1}{2} \nabla_{\varepsilon} w_{\varepsilon} \otimes \nabla_{\varepsilon} w_{\varepsilon}\right) \rightarrow \frac{1}{2} \int_{S} Q_{W}^{2}\left(\left(\begin{array}{cc}
\partial_{1} y_{1}+\frac{1}{2} w^{\prime 2} & E_{12}+\frac{1}{2} w^{\prime} \theta \\
E_{12}+\frac{1}{2} w^{\prime} \theta & E_{22}+\frac{1}{2} \theta^{2}
\end{array}\right)\right), \\
& \frac{1}{24} \int_{S} Q_{W}^{2}\left(\nabla_{\varepsilon}^{2} w_{\varepsilon}\right) \rightarrow \frac{1}{24} \int_{S} Q_{W}^{2}\left(\left(\begin{array}{cc}
w^{\prime \prime} & \theta^{\prime} \\
\theta^{\prime} & \gamma
\end{array}\right)\right) .
\end{aligned}
$$


This together with weak convergence (5.20), the expansion (5.5), and the positive definiteness on $\mathbb{R}_{\text {sym }}^{2 \times 2}$ of $Q_{W}^{2}$ shows that (5.20) holds with strong convergence.

\section{Proof of the main results}

In this section, we give the proofs of the main results.

\subsection{Passage from $2 \mathrm{D}$ to $1 \mathrm{D}$}

In this subsection, we prove Theorem 2.3. Let $M>0$. We fix a null sequence $\left(\varepsilon_{l}\right)_{l \in \mathbb{N}}$ and a sequence of initial data $\left(u_{\varepsilon_{l}}^{0}, v_{\varepsilon_{l}}^{0}\right) \in \mathscr{S}_{\varepsilon, M}^{2 D}$ such that $\left(u_{\varepsilon_{l}}^{0}, v_{\varepsilon_{l}}^{0}\right) \stackrel{\pi \sigma}{\longrightarrow}$ $\left(y^{0}, w^{0}, \theta^{0}\right) \in \mathscr{S}^{1 D}$ and $\phi_{\varepsilon}\left(u_{\varepsilon_{l}}^{0}, v_{\varepsilon_{l}}^{0}\right) \rightarrow \phi_{0}\left(y^{0}, w^{0}, \theta^{0}\right)$ as $l \rightarrow \infty$. Moreover, we assume that (H1) or (H2) holds.

Proof of Theorem 2.3(i). Let $\left(u_{\varepsilon_{l}}, v_{\varepsilon_{l}}\right)_{\varepsilon_{l}}$ be a sequence of curves of maximal slopes for $\phi_{\varepsilon_{l}}$ with respect to $\left|\partial \phi_{\varepsilon_{l}}\right|_{\mathcal{D}_{\varepsilon_{l}}}$ satisfying $\left(u_{\varepsilon_{l}}(0), v_{\varepsilon_{l}}(0)\right)=\left(u_{\varepsilon_{l}}^{0}, v_{\varepsilon_{l}}^{0}\right)$. We check that the assumptions of Theorem 3.3 are satisfied. The spaces $\left(\mathscr{S}_{\varepsilon_{l}, M}^{2 D}, \mathcal{D}_{\varepsilon_{l}}\right)$ and $\left(\mathscr{S}^{1 D}, \mathcal{D}_{0}\right)$ are complete metric spaces due to Lemma $4.1(\mathrm{i})$ and Lemma $4.2(\mathrm{i})$. In the notation of Subsection 3.2, we have $\mathscr{S}=\pi_{\varepsilon}\left(\mathscr{S}_{\varepsilon_{l}}^{2 D}\right)$ and $\mathscr{S}_{0}:=\mathscr{S}^{1 D}$. Clearly, we have $\mathscr{S}_{0} \subset \mathscr{S}$. Moreover, Proposition 2.1 yields (3.4) and Theorems 5.1, 5.2, and 5.6 give (3.3) and (3.5). Furthermore, the slopes are strong upper gradients due to Lemma 4.1(iii) and Lemma 4.3(ii). As the energies of the curves of maximal slopes are uniformly bounded depending only on the initial data, see (3.6), we can apply Theorem 3.3. This yields the existence of a curve of maximal slope $(y, w, \theta)$ for $\phi_{0}$ with respect to $\left|\partial \phi_{0}\right|_{\mathcal{D}_{0}}$ and the convergence $\left(u_{\varepsilon_{l}}(t), v_{\varepsilon_{l}}(t)\right) \stackrel{\pi \sigma}{\rightarrow}(y(t), w(t), \theta(t))$ for $t \geq 0$, up to a subsequence. It remains to observe that the convergence is actually strong. This follows from the fact that $\lim _{l \rightarrow \infty} \phi_{\varepsilon_{l}}\left(u_{\varepsilon_{l}}(t), v_{\varepsilon_{l}}(t)\right)=\phi_{0}(y(t), w(t), \theta(t))$ for $t \geq 0$ (see Theorem 3.3) and Lemma 5.8.

Proof of Theorem 2.3(iii). Let $\left(\tau_{l}\right)_{l}$ be a null sequence and let $\bar{Y}_{\varepsilon_{l}, \tau_{l}}$ be a discrete solution to the two-dimensional problem. As in the previous proof, we check that all assumptions of Theorem 3.2 are satisfied. Thus, there exists a subsequence such that we have

$$
\bar{Y}_{\varepsilon_{l}, \tau_{l}}(t) \stackrel{\pi \sigma}{\longrightarrow}(y(t), w(t), \theta(t))
$$

for all $t \geq 0$ as $l \rightarrow \infty$ and $(y, w, \theta)$ is a curve of maximal slope for $\phi_{0}$ with respect to $\left|\partial \phi_{0}\right|_{\mathcal{D}_{0}}$. Moreover, the convergence holds in a strong sense for all $t \geq 0$ due to Lemma 5.8.

Proof of Theorem 2.3(ii). Let $\tau>0$ and let $\bar{Y}_{\varepsilon_{l}, \tau}$ be a discrete solution to the two-dimensional problem with $\bar{Y}_{\varepsilon_{l}, \tau}(0)=\left(u_{\varepsilon_{l}}^{0}, v_{\varepsilon_{l}}^{0}\right)$. By construction, the energies of the discrete solution $\bar{Y}_{\varepsilon_{l}, \tau}$ are uniformly bounded depending only on the initial data. Thus, by a diagonal argument and Proposition 2.1, we obtain a subsequence and $\left(U_{\tau}^{n}\right)_{n \in \mathbb{N}}$ such that $\bar{Y}_{\varepsilon_{l}, \tau}(n \tau) \stackrel{\pi \sigma}{\rightarrow} U_{\tau}^{n}$ for all $n \in \mathbb{N}$. Now, we need to show that 

(i) $U_{\tau}^{n}=\underset{v \in \mathscr{S}^{1 D}}{\arg \min } \boldsymbol{\Phi}_{\mathbf{0}}\left(\tau, U_{\tau}^{n-1} ; v\right)$,
(ii) $\bar{Y}_{\varepsilon_{l}, \tau}(n \tau) \stackrel{\pi \rho}{\rightarrow} U_{\tau}^{n}$,
(iii) $\phi_{\varepsilon_{l}}\left(\bar{Y}_{\varepsilon_{l}, \tau}(n \tau)\right) \rightarrow \phi_{0}\left(U_{\tau}^{n}\right)$

for all $n \in \mathbb{N}$. We show properties (i)-(iii) by induction. Suppose that $\bar{Y}_{\varepsilon_{l}, \tau}((n-$ $1) \tau) \stackrel{\pi \rho}{\rightarrow} U_{\tau}^{n-1}$ and $\phi_{\varepsilon_{l}}\left(\bar{Y}_{\varepsilon_{l}, \tau}((n-1) \tau)\right) \rightarrow \phi_{0}\left(U_{\tau}^{n-1}\right)$ for a fixed $n \in \mathbb{N}$. (Clearly, this holds for $n=1$ by Lemma 5.8 as $\bar{Y}_{\varepsilon_{l}, \tau}(0) \stackrel{\pi \sigma}{\longrightarrow} U_{\tau}^{0}$ and $\phi_{\varepsilon_{l}}\left(\bar{Y}_{\varepsilon_{l}, \tau}(0)\right) \rightarrow$ $\phi_{0}\left(U_{\tau}^{0}\right)$ by assumption.) Due to Theorem 5.7, the element $U_{\tau}^{n}$ is a minimizer of $\boldsymbol{\Phi}_{\mathbf{0}}\left(\tau, U_{\tau}^{n-1} ; \cdot\right)$ which shows (i). Moreover, Theorem 5.7 also yields $\phi_{\varepsilon_{l}}\left(\bar{Y}_{\varepsilon_{l}, \tau}(n \tau)\right) \rightarrow \phi_{0}\left(U_{\tau}^{n}\right)$ which gives (iii). Finally, (ii) follows by Lemma 5.8. By defining $\bar{U}_{\tau}$ as in (3.2) we can conclude.

\subsection{Solutions in $1 \mathrm{D}$}

In this subsection we prove Theorem 2.2.

Proof of Theorem 2.2(i). Our goal is to apply Theorem 3.2: instead of a sequence of metric spaces, we only consider the single metric space $\left(\mathscr{S}^{1 D}, \mathcal{D}_{0}\right)$ which is complete due to Lemma 4.2(i). We let $\sigma$ be the weak convergence in $\left(\mathscr{S}^{1 D},\|\cdot\|_{\text {can }}\right)$, for which Lemma 4.2 (iii) provides the compactness property (3.4). By Lemma 4.2(iv) and Lemma 4.3(iii) we get (3.3) and (3.5). As $\left|\partial \phi_{0}\right|_{\mathcal{D}_{0}}$ is a strong upper gradient, see Lemma $4.3(\mathrm{ii})$, Theorem 3.2 yields the convergence of time-discrete solution to a curve of maximal slope. Strong convergence with respect to $\|\cdot\|_{\text {can }}$ can be obtained by repeating the arguments in the proof of Lemma 5.8. We omit the details. Eventually, convergence with respect to $\mathcal{D}_{0}$ is induced by Lemma 4.2 (ii).

Remark 6.1. (Alternative existence proof) If one is only interested in existence and not in time-discrete approximation, one could directly use Theorem 2.3(i): by Theorem 5.1 we can construct a recovery sequence $\left(u_{\varepsilon}^{0}, v_{\varepsilon}^{0}\right)_{\varepsilon}$ such that $\left(u_{\varepsilon}^{0}, v_{\varepsilon}^{0}\right) \stackrel{\pi \rho}{\rightarrow}\left(y^{0}, w^{0}, \theta^{0}\right)$. Then, curves of maximal slope $\left(u_{\varepsilon}, v_{\varepsilon}\right)$ for $\phi_{\varepsilon}$ with respect to $\left|\partial \phi_{\varepsilon}\right|_{\mathcal{D}_{\varepsilon}}$ in the two-dimensional setting exist by [22, Theorem 2.2], up to minor adjustments, and by Theorem 2.3(i) we conclude the proof. Note, however, that in this way we need to require $(\mathbf{H 1})$ or $(\mathbf{H 2})$.

After having shown existence of curves of maximal slope, our goal is to establish a relation to the effective one-dimensional equations, see Theorem 2.2(ii). The natural idea is to make use of the energy identity (3.6). For this purpose, we use a finer representation of the local slope $\left|\partial \phi_{0}\right|_{\mathcal{D}_{0}}$. Afterwards, we give sharp estimates for the metric derivative and the derivative of $\phi_{0} \circ(y, w, \theta)$. This will provide enough information for the relation to the equations. For the following arguments, it is convenient to introduce the abbreviations

$$
\begin{aligned}
& H(y, w, \theta \mid \hat{w}):=\left(\partial_{1} y_{1}+w^{\prime} \hat{w}^{\prime}, w^{\prime \prime}, \theta^{\prime}\right) \in L^{2}\left(S ; \mathbb{R}^{3}\right), \\
& G(y, w, \theta):=\left(\partial_{1} y_{1}+\frac{\left|w^{\prime}\right|^{2}}{2}, w^{\prime \prime}, \theta^{\prime}\right) \in L^{2}\left(S ; \mathbb{R}^{3}\right)
\end{aligned}
$$


for $(y, w, \theta) \in \mathscr{S}^{1 D}$ and $\hat{w} \in W^{2,2}(I)$. By an elementary computation we get

$$
\begin{aligned}
G(y, w, \theta)-G(\tilde{y}, \tilde{w}, \tilde{\theta})= & \left(\partial_{1} y_{1}-\partial_{1} \tilde{y}_{1}+\left|w^{\prime}\right|^{2}-\tilde{w}^{\prime} w^{\prime}, w^{\prime \prime}-\tilde{w}^{\prime \prime}, \theta^{\prime}-\tilde{\theta}^{\prime}\right) \\
& -\left(\frac{\left|w^{\prime}\right|^{2}}{2}-w^{\prime} \tilde{w}^{\prime}+\frac{\left|\tilde{w}^{\prime}\right|^{2}}{2}, 0,0\right) \\
= & H(y-\tilde{y}, w-\tilde{w}, \theta-\tilde{\theta} \mid w)-\frac{1}{2}\left(\left(w^{\prime}-\tilde{w}^{\prime}\right)^{2}, 0,0\right) .
\end{aligned}
$$

We further introduce extended quadratic forms

$$
\bar{Q}_{S}\left(x_{1}, x_{2}, x_{3}\right):=Q_{S}^{0}\left(x_{1}\right)+\frac{1}{12} Q_{S}^{1}\left(x_{2}, x_{3}\right)
$$

for $\left(x_{1}, x_{2}, x_{3}\right)^{T} \in \mathbb{R}^{3}$ and $S=W, R$.

Lemma 6.2. (Representation of the energy and the metric) For $(y, w, \theta),(\tilde{y}, \tilde{w}$, $\tilde{\theta}) \in \mathscr{S}^{1 D}$, it holds

$$
\begin{aligned}
\phi_{0}(y, w, \theta) & =\frac{1}{2} \int_{S} \bar{Q}_{W}(G(y, w, \theta)) \\
\mathcal{D}_{0}^{2}((y, w, \theta),(\tilde{y}, \tilde{w}, \tilde{\theta})) & =\int_{S} \bar{Q}_{R}(G(y, w, \theta)-G(\tilde{y}, \tilde{w}, \tilde{\theta})) .
\end{aligned}
$$

Proof. The statement follows directly from (2.15)-(2.16) and (6.1).

By (2.10) and (2.11) we find that $\bar{Q}_{S}$ is positive definite on $\mathbb{R}^{3}$ for $S=$ $W, R$. We denote by $\overline{\mathbb{C}}_{S}$ its associated bilinear form which induces a bijective mapping $\left(x_{1}, x_{2}, x_{3}\right) \mapsto \overline{\mathbb{C}}_{S}\left(x_{1}, x_{2}, x_{3}\right)$ from $\mathbb{R}^{3}$ to $\mathbb{R}^{3}$. By $\sqrt{\overline{\mathbb{C}}_{S}}$ we denote its unique root and by $\sqrt{\overline{\mathbb{C}}_{S}}-1$ the inverse of $\sqrt{\overline{\mathbb{C}}_{S}}$.

Lemma 6.3. (Fine representation of the one-dimensional slope) There exists a differential operator $\mathcal{L}: \mathscr{S}^{1 D} \rightarrow L^{2}\left(S ; \mathbb{R}^{3}\right)$ satisfying

$$
\int_{S} \mathcal{L}(y, w, \theta) \cdot H\left(\phi_{y}, \phi_{w}, \phi_{\theta} \mid w\right)=0
$$

for all $(y, w, \theta) \in \mathscr{S}^{1 D}$ and $\left(\phi_{y}, \phi_{w}, \phi_{\theta}\right) \in B N_{(0,0)}\left(S ; \mathbb{R}^{2}\right) \times W_{0}^{2,2}(I) \times W_{0}^{1,2}(I)$ such that the local slope at $(y, w, \theta) \in \mathscr{S}^{1 D}$ can be represented by

$$
\left|\partial \phi_{0}\right|_{\mathcal{D}_{0}}(y, w, \theta)=\left\|\sqrt{\overline{\mathbb{C}}_{R}^{-1}}\left(\overline{\mathbb{C}}_{W} G(y, w, \theta)+\mathcal{L}(y, w, \theta)\right)\right\|_{L^{2}\left(S ; \mathbb{R}^{3}\right)} .
$$

The proof follows along the lines of the corresponding representation in dimension two, see [22, Lemma 6.1]. For the convenience of the reader, we give a self-contained proof in Appendix A.

Proof of Theorem 2.2(ii). We now use a standard technique to relate curves of maximal slope to PDEs in Hilbert spaces, see [4, Section 1.4]. More precisely, the proof follows the lines of [22, Theorem 2.2(ii)]. We divide the proof into three steps. First, we construct the curve $\left(\xi_{1}, \xi_{2}, w, \theta\right)$ and prove the regularity stated in the theorem. Step 2 consists in deriving sharp estimates for $\left|v^{\prime}\right|_{\mathcal{D}_{0}}$ and $\frac{d}{d t} \phi_{0} \circ v$ for $v=(y, w, \theta)$. This allows to relate the curve to the one-dimensional system of equations in Step 3. 
Step 1: Let $(y, w, \theta):[0, \infty) \rightarrow \mathscr{S}^{1 D}$ be a curve of maximal slope for $\phi_{0}$ with respect to $\left|\partial \phi_{0}\right|_{\mathcal{D}_{0}}$. Using $(2.9)$ there exist functions $\xi_{1}:[0, \infty) \rightarrow W_{\hat{u}_{1}}^{1,2}(I)$ and $\xi_{2}:[0, \infty) \rightarrow W_{\hat{u}_{2}}^{2,2}(I)$ such that $y_{1}(t)(x)=\xi_{1}(t)\left(x_{1}\right)-x_{2} \xi_{2}(t)^{\prime}\left(x_{1}\right)$ and $y_{2}(t)(x)=\xi_{2}(t)\left(x_{1}\right)$ for all $t \geq 0$ almost everywhere in $S$. By definition, we have for every $t \geq 0$

$$
\left\|\xi_{1}(t)\right\|_{W^{1,2}(I)}+\left\|\xi_{2}(t)\right\|_{W^{2,2}(I)} \leq C\|y(t)\|_{W^{1,2}\left(S ; \mathbb{R}^{2}\right)}
$$

As $(y, w, \theta)$ is a curve of maximal slope we get that $\phi_{0}(y(t), w(t), \theta(t))$ is decreasing in time, see (3.6). This together with Lemma 4.2(iii) and (6.4) yields

$$
\left(\xi_{1}, \xi_{2}, w, \theta\right) \in L^{\infty}([0, \infty) ; \mathcal{K})
$$

where $\mathcal{K}$ is defined in (2.12). As $(y(t), w(t), \theta(t))_{t>0}$ is absolutely continuous with respect to $\left(\mathscr{S}^{1 D}, \mathcal{D}_{0}\right)$ we have that $\left|(y, w, \theta)^{\prime}\right|_{\mathcal{D}_{0}} \in L^{2}([0, \infty))$. Then, by Lemma 4.2(ii), (6.4), and (6.5) we observe that $\left(\xi_{1}, \xi_{2}, w, \theta\right)$ is an absolutely continuous curve with respect to $\mathcal{K}$. Thus, by [4, Remark 1.1.3] we observe that $\xi_{1}, \xi_{2}, w$, and $\theta$ are differentiable for a.e. $t$ and we have (2.17). More precisely, for all $0 \leq s<t$, and almost everywhere in $S$ (respectively $I$ ) it holds that

$$
f(t)-f(s)=\int_{s}^{t} \partial_{t} f(r) \mathrm{d} r \quad \text { for } f \in\left\{\partial_{1} y_{1}, w^{\prime}, w^{\prime \prime}, \theta^{\prime}\right\} .
$$

Step 2: As preparation for the representation of the metric derivative, we now consider the difference $G(y, w, \theta)(t)-G(y, w, \theta)(s)$. The identity (6.2) and the linearity of $H(\cdot, \cdot, \cdot \mid w(t))$ yield for a.e. $t$ and a.e. $x \in S$

$$
\begin{aligned}
\lim _{s \rightarrow t} \frac{G(y, w, \theta)(t)-G(y, w, \theta)(s)}{t-s}= & H\left(\partial_{t} y(t), \partial_{t} w(t), \partial_{t} \theta(t) \mid w(t)\right) \\
& -\partial_{t} w(t) \lim _{s \rightarrow t} \frac{1}{2}(w(t)-w(s)) \\
= & H\left(\partial_{t} y(t), \partial_{t} w(t), \partial_{t} \theta(t) \mid w(t)\right) .
\end{aligned}
$$

Using (6.1), (6.6), Poincaré's and Jensen's inequality, and Fubini's Theorem, we obtain for all $0 \leq s \leq t$

$$
\begin{aligned}
& \left\|G(y(t), w(t), \theta(t))-G(y(s), w(s), \theta(s))-\int_{s}^{t} H\left(\partial_{t} y(r), \partial_{t} w(r), \partial_{t} \theta(r) \mid w(t)\right) \mathrm{d} r\right\|_{L^{2}\left(S ; \mathbb{R}^{3}\right)}^{2} \\
& \quad=\int_{S} \frac{1}{2}\left(w^{\prime}(t)-w^{\prime}(s)\right)^{4} \leq C\left(\int_{S}\left(w^{\prime \prime}(t)-w^{\prime \prime}(s)\right)^{2}\right)^{2} \\
& \quad=C\left(\int_{S}|t-s|^{2}\left(\frac{1}{|t-s|} \int_{s}^{t} \partial_{t} w^{\prime \prime}(r) \mathrm{d} r\right)^{2} \mathrm{~d} x\right)^{2} \\
& \quad \leq C\left(\int_{S}|t-s| \int_{s}^{t} \partial_{t} w^{\prime \prime}(r)^{2} \mathrm{~d} r \mathrm{~d} x\right)^{2} \\
& \quad=C|t-s|^{2}\left(\int_{s}^{t}\left\|\partial_{t} w^{\prime \prime}(r)\right\|_{L^{2}(S)}^{2}\right)^{2} .
\end{aligned}
$$

We now estimate the metric derivative $\left|(y, w, \theta)^{\prime}\right|_{\mathcal{D}_{0}}$. By Lemma 6.2, (6.7), and Fatou's Lemma we get for a.e. $t \geq 0$ 


$$
\begin{aligned}
\left|(y, w, \theta)^{\prime}\right|_{\mathcal{D}_{0}}(t) & =\lim _{s \rightarrow t}\left(\frac{\mathcal{D}_{0}^{2}((y(t), w(t), \theta(t)),(y(s), w(s), \theta(s)))}{|t-s|^{2}}\right)^{1 / 2} \\
& \geq\left(\int_{S} \liminf _{s \rightarrow t} \bar{Q}_{R}\left(\frac{G(y(t), w(t), \theta(t))-G(y(s), w(s), \theta(s))}{|t-s|}\right)\right)^{1 / 2} \\
& =\left\|\sqrt{\overline{\mathbb{C}}_{R}} H\left(\partial_{t} y(t), \partial_{t} w(t), \partial_{t} \theta(t) \mid w(t)\right)\right\|_{L^{2}\left(S ; \mathbb{R}^{3}\right)}
\end{aligned}
$$

We now analyze the derivative $\frac{\mathrm{d}}{\mathrm{d} t}\left(\phi_{0} \circ(y, w, \theta)\right)(t)$ of the absolutely continuous curve $\phi_{0} \circ(y, w, \theta)$. Note that for a.e. $t \geq 0$ we have $\lim _{s \rightarrow t} \int_{s}^{t}\left\|\partial_{t} w^{\prime \prime}(r)\right\|_{L^{2}(S)}^{2} \mathrm{~d} r$ $=0$ by (2.17) and, in a similar fashion, $\lim _{s \rightarrow t}|s-t|^{-1} \| \int_{s}^{t} H\left(\partial_{t} y(r), \partial_{t} w(r)\right.$, $\left.\partial_{t} \theta(r) \mid w(t)\right) \mathrm{d} r \|_{L^{2}\left(S ; \mathbb{R}^{3}\right)}^{2}=0$ by (2.17) and Hölder's inequality. Thus, using Lemma 6.2, (5.5), and (6.7)- (6.8), we obtain for a.e. $t \geq 0$

$$
\begin{aligned}
\frac{\mathrm{d}}{\mathrm{d} t}\left(\phi_{0} \circ(y, w, \theta)\right)(t)= & \lim _{s \rightarrow t} \frac{\phi_{0}(y(t), w(t), \theta(t))-\phi_{0}(y(s), w(s), \theta(s))}{t-s} \\
\geq & \liminf _{s \rightarrow t} \frac{1}{t-s} \int_{S} \overline{\mathbb{C}}_{W}[G(y(t), w(t), \theta(t)), G(y(t), w(t), \theta(t) \\
& -G(y(s), w(s), \theta(s))] \\
& -\limsup _{s \rightarrow t} \frac{1}{2(t-s)} \int_{S} \bar{Q}_{W}(G(y(t), w(t), \theta(t)) \\
& -G(y(s), w(s), \theta(s))) \\
\geq & \int_{S} \overline{\mathbb{C}}_{W}\left[G(y(t), w(t), \theta(t)), H\left(\partial_{t} y(t), \partial_{t} w(t), \partial_{t} \theta(t) \mid w(t)\right)\right] .
\end{aligned}
$$

By the property of $\mathcal{L}$ stated in Lemma 6.3, and the fact that $\partial_{t} y(t), \partial_{t} w(t), \partial_{t} \theta(t)$ vanish on $\partial I$ and $\partial S$, respectively, we get

$$
\begin{aligned}
\frac{\mathrm{d}}{\mathrm{d} t}\left(\phi_{0} \circ(y, w, \theta)\right)(t) \geq & \int_{S}\left(\overline{\mathbb{C}}_{W} G(y(t), w(t), \theta(t))\right. \\
& +\mathcal{L}(y(t), w(t), \theta(t))) \cdot H\left(\partial_{t} y(t), \partial_{t} w(t), \partial_{t} \theta(t) \mid w(t)\right) \\
= & \int_{S} \sqrt{\overline{\mathbb{C}}_{R}}-1\left(\overline{\mathbb{C}}_{W} G(y(t), w(t), \theta(t))\right. \\
& +\mathcal{L}(y(t), w(t), \theta(t))) \cdot \sqrt{\overline{\mathbb{C}}_{R}} H\left(\partial_{t} y(t), \partial_{t} w(t), \partial_{t} \theta(t) \mid w(t)\right) .
\end{aligned}
$$

We find by Lemma 6.3, (6.9), and Young's inequality

$$
\begin{aligned}
\frac{\mathrm{d}}{\mathrm{d} t} \phi_{0}((y, w, \theta)(t)) & \geq-\frac{\left|(y, w, \theta)^{\prime}\right|_{\mathcal{D}_{0}}^{2}(t)}{2}-\frac{\left|\partial \phi_{0}\right|_{\mathcal{D}_{0}}^{2}(y(t), w(t), \theta(t))}{2} \\
& \geq \frac{\mathrm{d}}{\mathrm{d} t} \phi_{0}((y, w, \theta)(t))
\end{aligned}
$$

for a.e. $t \geq 0$, where the last step is a consequence of the fact that $(y(t), w(t), \theta(t))$ is a curve of maximal slope with respect to $\phi_{0}$. Consequently, all inequalities employed in the proof are in fact equalities, and we get 


$$
\begin{aligned}
& {\sqrt{\overline{\mathbb{C}}_{R}}-1}^{-1}\left(\overline{\mathbb{C}}_{W} G(y(t), w(t), \theta(t))+\mathcal{L}(y(t), w(t), \theta(t))\right) \\
& \quad+\sqrt{\overline{\mathbb{C}}_{R}} H\left(\partial_{t} y(t), \partial_{t} w(t), \partial_{t} \theta(t) \mid w(t)\right)=0
\end{aligned}
$$

pointwise a.e. in $S$ for a.e. $t \geq 0$. Multiplying the equation with $\sqrt{\overline{\mathbb{C}}_{R}}$ from the left and testing with $H\left(\phi_{y}, \phi_{w}, \phi_{\theta} \mid w(t)\right)$ from the right for $\left(\phi_{y}, \phi_{w}, \phi_{\theta}\right) \in$ $B N_{(0,0)}\left(S, \mathbb{R}^{2}\right) \times W_{0}^{2,2}(I) \times W_{0}^{1,2}(I)$ yields with the property of Lemma 6.3 for a.e. $t \geq 0$

$$
\begin{aligned}
& \int_{S}\left(\overline{\mathbb{C}}_{W} G(y(t), w(t), \theta(t))+\overline{\mathbb{C}}_{R} H\left(\partial_{t} y(t), \partial_{t} w(t), \partial_{t} \theta(t) \mid w(t)\right)\right) \\
& \cdot H\left(\phi_{y}, \phi_{w}, \phi_{\theta} \mid w(t)\right)=0 .
\end{aligned}
$$

Step 3: Eventually, we verify that $\left(\xi_{1}, \xi_{2}, w, \theta\right)$ solve the one-dimensional equations. To this end, we use the identity $(6.10)$ by choosing functions such that $\phi_{i}=\phi_{j}=0$ for $i, j \in\{y, w, \theta\}$ with $i \neq j$ in (6.10). The simplest case is the derivation of (2.13d) by setting $\phi_{y}=0$ and $\phi_{w}=0$. To this end, we recall (6.1) and $(6.3)$ and remark that $\partial_{i} \bar{Q}_{S}(\cdot)=2\left(\overline{\mathbb{C}}_{S}(\cdot)\right)_{i}$ as $\overline{\mathbb{C}}_{S}$ is symmetric. Thus, an integration leads to (omitting the variable $t$ from now on)

$$
\begin{aligned}
0 & =\int_{S}\left(\overline{\mathbb{C}}_{W} G(y, w, \theta)+\overline{\mathbb{C}}_{R} H\left(\partial_{t} y, \partial_{t} w, \partial_{t} \theta \mid w\right)\right) \cdot\left(0,0, \phi_{\theta}^{\prime}\right) \\
& =\frac{1}{24} \int_{I}\left(\partial_{2} Q_{W}^{1}\left(w^{\prime \prime}, \theta^{\prime}\right)+\partial_{2} Q_{R}^{1}\left(\partial_{t} w^{\prime \prime}, \partial_{t} \theta^{\prime}\right)\right) \phi_{\theta}^{\prime},
\end{aligned}
$$

which is exactly equation (2.13d). Setting $\phi_{y}=0$ and $\phi_{\theta}=0$ leads to

$$
\begin{aligned}
0=\int_{I} & \left(C_{W}^{0}\left(\xi_{1}^{\prime}+\frac{\left|w^{\prime}\right|^{2}}{2}\right)+C_{R}^{0}\left(\partial_{t} \xi_{1}^{\prime}+w^{\prime} \partial_{t} w^{\prime}\right)\right) w^{\prime} \phi_{w}^{\prime} \\
& +\frac{1}{24} \int_{I}\left(\partial_{1} Q_{W}^{1}\left(w^{\prime \prime}, \theta^{\prime}\right)+\partial_{1} Q_{R}^{1}\left(\partial_{t} w^{\prime \prime}, \partial_{t} \theta^{\prime}\right)\right) \phi_{w}^{\prime \prime},
\end{aligned}
$$

where we used (2.9) and $\int_{-1 / 2}^{1 / 2} x_{2} \mathrm{~d} x_{2}=0$. This gives (2.13c). The missing equations follow by setting $\phi_{\theta}=0$ and $\phi_{w}=0$ : characterization (2.9) yields the existence of functions $\phi_{\xi_{1}} \in W_{0}^{1,2}(I)$ and $\phi_{\xi_{2}} \in W_{0}^{2,2}(I)$ such that $\partial_{1}\left(\phi_{y}\right)_{1}=$ $\phi_{\xi_{1}}^{\prime}-x_{2} \phi_{\xi_{2}}^{\prime \prime}$. Inserting this and using $\int_{-1 / 2}^{1 / 2} x_{2}^{2} \mathrm{~d} x_{2}=1 / 12$ yields

$0=\int_{I}\left(C_{W}^{0}\left(\xi_{1}^{\prime}+\frac{\left|w^{\prime}\right|}{2}\right)+C_{R}^{0}\left(\partial_{t} \xi_{1}^{\prime}+w^{\prime} \partial_{t} w^{\prime}\right)\right) \phi_{\xi_{1}}^{\prime}+\frac{1}{12}\left(C_{W}^{0} \xi_{2}^{\prime \prime}+C_{R}^{0} \partial_{t} \xi_{2}^{\prime \prime}\right) \phi_{\xi_{2}}^{\prime \prime}$.

As we can choose $\phi_{\xi_{1}}=0$ and $\phi_{\xi_{2}}=0$ independently, we obtain (2.13a) and (2.13b).

\section{Acknowledgements}

This work was funded by the DFG project FR 4083/5-1 and by the Deutsche Forschungsgemeinschaft (DFG, German Research Foundation) under Germany's Excellence Strategy EXC 2044 -390685587, Mathematics Münster: DynamicsGeometry-Structure. 
Funding Open Access funding enabled and organized by Projekt DEAL.

Open Access. This article is licensed under a Creative Commons Attribution 4.0 International License, which permits use, sharing, adaptation, distribution and reproduction in any medium or format, as long as you give appropriate credit to the original author(s) and the source, provide a link to the Creative Commons licence, and indicate if changes were made. The images or other third party material in this article are included in the article's Creative Commons licence, unless indicated otherwise in a credit line to the material. If material is not included in the article's Creative Commons licence and your intended use is not permitted by statutory regulation or exceeds the permitted use, you will need to obtain permission directly from the copyright holder. To view a copy of this licence, visit http://creativecommons. org/licenses/by/4.0/.

Publisher's Note Springer Nature remains neutral with regard to jurisdictional claims in published maps and institutional affiliations.

\section{Appendix A. One-dimensional properties}

In this section we give the proofs of the properties in the one-dimensional setting. The arguments are similar to the two-dimensional case and one can follow closely the lines of $[21,22]$. Yet, we include complete proofs here for the reader's convenience. We start by proving Lemma 4.2 which is rather elementary. The proof of Lemma 4.3 is more technical and is divided into the following steps: by constructing suitable "generalized geodesics", see Lemma A.1, we establish the representation of the local slope $\left|\partial \phi_{0}\right|_{\mathcal{D}_{0}}$ stated in Lemma 4.3(i). This is at the core of proving Lemma 4.3(ii),(iii), where we additionally construct suitable mutual recovery sequences for the weak lower semicontinuity of the slope similar to Lemma 5.3 (see Lemma A.2). Finally, at the end of the section, we give the proof of Lemma 6.3. In the following, $C>0$ denotes a universal constant that may change from line to line. Moreover, the symbol $\rightarrow$ will stand for weak convergence in the space $\left(\mathscr{S}^{1 D},\|\cdot\|_{\text {can }}\right)$.

Proof of Lemma 4.2. We first derive the lower bounds (4.1) and (4.2). By Poincaré's inequality and the fact that $Q_{R}^{1}$ is positive definite, we get for all $(y, w, \theta),(\tilde{y}, \tilde{w}, \tilde{\theta}) \in \mathscr{S}^{1 D}$ that

$$
\begin{aligned}
\|w-\tilde{w}\|_{W^{2,2}(I)}^{2}+\|\theta-\tilde{\theta}\|_{W^{1,2}(I)}^{2} & \leq C\left\|w^{\prime \prime}-\tilde{w}^{\prime \prime}\right\|_{L^{2}(I)}^{2}+C\left\|\theta^{\prime}-\tilde{\theta}^{\prime}\right\|_{L^{2}(I)}^{2} \\
& \leq C \int_{I} Q_{R}^{1}\left(w^{\prime \prime}-\tilde{w}^{\prime \prime}, \theta^{\prime}-\tilde{\theta}^{\prime}\right) \\
& \leq C \mathcal{D}_{0}^{2}((y, w, \theta),(\tilde{y}, \tilde{w}, \tilde{\theta}))
\end{aligned}
$$

i.e., (4.1) holds. By (2.9) and Korn-Poincaré's inequality we get

$$
\|y-\tilde{y}\|_{W^{1,2}\left(S ; \mathbb{R}^{2}\right)}^{2} \leq C\|e(y-\tilde{y})\|_{L^{2}\left(S ; \mathbb{R}_{\mathrm{sym}}^{2 \times 2}\right)}^{2}=C\left\|\partial_{1} y_{1}-\partial_{1} \tilde{y}_{1}\right\|_{L^{2}(S)}^{2} .
$$


This along with the triangle inequality, the positivity of $Q_{R}^{0}$, and Hölder's inequality yields

$$
\begin{array}{rl}
\|y-\tilde{y}\|_{W^{1,2}\left(S ; \mathbb{R}^{2}\right) \leq}^{2} & C \int_{S} Q_{R}^{0}\left(\partial_{1} y_{1}-\partial_{1} \tilde{y}_{1}+\frac{\left|w^{\prime}\right|^{2}}{2}-\frac{\left|\tilde{w}^{\prime}\right|^{2}}{2}\right) \\
& +C\left\|\left|w^{\prime}\right|^{2}-\left|\tilde{w}^{\prime}\right|^{2}\right\|_{L^{2}(I)}^{2} \\
\leq & C \mathcal{D}_{0}^{2}((y, w, \theta),(\tilde{y}, \tilde{w}, \tilde{\theta}))+C\left\|w^{\prime}+\tilde{w}^{\prime}\right\|_{L^{4}(I)}^{2}\left\|w^{\prime}-\tilde{w}^{\prime}\right\|_{L^{4}(I)}^{2} .
\end{array}
$$

This shows (4.2). Thus, the embedding $W^{1,2}(I) \subset \subset L^{4}(I)$ and Hölder's inequality yield that every converging sequence with respect to the topology induced by $\mathcal{D}_{0}$ converges with respect to $\|\cdot\|_{\text {can }}$ and vice versa. This shows (ii). Using analogous computations to (ii), with $\phi_{0}$ in place of $\mathcal{D}_{0}^{2}$, we find that (iii) holds. To see (iv), we consider $\left(y_{k}, w_{k}, \theta_{k}\right) \rightarrow(y, w, \theta)$ and $\left(\tilde{y}_{k}, \tilde{w}_{k}, \tilde{\theta}_{k}\right) \rightarrow$ $(\tilde{y}, \tilde{w}, \tilde{\theta})$. As $W^{1,2}(I) \subset \subset L^{4}(I)$, we obtain

$\partial_{1}\left(y_{k}\right)_{1}+\frac{\left|w_{k}^{\prime}\right|^{2}}{2} \rightarrow \partial_{1} y_{1}+\frac{\left|w^{\prime}\right|^{2}}{2}, \quad \partial_{1}\left(\tilde{y}_{k}\right)_{1}+\frac{\left|\tilde{w}_{k}^{\prime}\right|^{2}}{2} \rightarrow \partial_{1} \tilde{y}_{1}+\frac{\left|\tilde{w}^{\prime}\right|^{2}}{2} \quad$ in $L^{2}(S)$.

As the quadratic forms $Q_{W}^{0}, Q_{W}^{1}, Q_{R}^{0}$, and $Q_{R}^{1}$ are positive definite, weak lower semicontinuity follows. We finally prove (i). The positivity and the completeness follow from (ii). Eventually, the triangle inequality follows from the fact that $\mathcal{D}_{0}^{2}$ is the sum of two quadratic forms.

We now aim at proving that $\left|\partial \phi_{0}\right|_{\mathcal{D}_{0}}$ is weakly lower semicontinuous and a strong upper gradient. To verify this, we follow the approach of [23] which is based on a generalized convexity condition as the metric $\mathcal{D}_{0}$ and the energy $\phi_{0}$ are non-convex, due to the nonlinearity $\left|w^{\prime}\right|^{2}$. We refer to [23, Remark 1] for a detailed discussion. In the sequel, we frequently replace $(y, w, \theta)$ by a single variable $u$ for notational convenience.

Lemma A.1. (Convexity and generalized geodesics in the one-dimensional setting) Let $M>0$. Let $\Phi^{1}(t):=\sqrt{t^{2}+C t^{3}+C t^{4}}$ and $\Phi_{M}^{2}(t):=C \sqrt{M} t^{2}+$ $C t^{3}+C t^{4}$ for any $C>0$ large enough. Then, for all $u_{0}:=\left(y_{0}, w_{0}, \theta_{0}\right) \in \mathscr{S}^{1 D}$ satisfying $\phi_{0}\left(u_{0}\right) \leq M$ and all $u_{1}:=\left(y_{1}, w_{1}, \theta_{1}\right) \in \mathscr{S}^{1 D}$ we have

(i) $\mathcal{D}_{0}\left(u_{0}, u_{s}\right) \leq s \Phi^{1}\left(\mathcal{D}_{0}\left(u_{0}, u_{1}\right)\right)$

(ii) $\phi_{0}\left(u_{s}\right) \leq(1-s) \phi_{0}\left(u_{0}\right)+s \phi_{0}\left(u_{1}\right)+s \Phi_{M}^{2}\left(\mathcal{D}_{0}\left(u_{0}, u_{1}\right)\right)$

for $u_{s}:=(1-s) u_{0}+s u_{1}$ and $s \in[0,1]$.

Proof. Let $M>0, u_{0} \in \mathscr{S}^{1 D}$ with $\phi_{0}\left(u_{0}\right) \leq M$, and $u_{1} \in \mathscr{S}^{1 D}$. For convenience, we first introduce some abbreviations and provide some preliminary estimates. We set $\mathcal{D}=\mathcal{D}_{0}\left(u_{0}, u_{1}\right), B_{\text {diff }}=\frac{1}{2}\left(w_{0}^{\prime}-w_{1}^{\prime}\right)^{2}$, and $G_{0}^{s}=\partial_{1}\left(y_{s}\right)_{1}+\frac{1}{2}\left|w_{s}^{\prime}\right|^{2}$ for $s \in[0,1]$. Then, by Sobolev embedding and (4.1) we find

$$
\left\|B_{\text {diff }}\right\|_{L^{2}(I)} \leq C\left\|w_{0}^{\prime}-w_{1}^{\prime}\right\|_{L^{4}(I)}^{2} \leq C\left\|w_{0}-w_{1}\right\|_{W^{2,2}(I)}^{2} \leq C \mathcal{D}^{2} .
$$

Then, by the positivity of $Q_{R}^{0}$ and the definition of $\mathcal{D}_{0}$ we derive

$$
\left\|G_{0}^{1}-G_{0}^{0}\right\|_{L^{2}(S)}^{2} \leq C \int_{S} Q_{R}^{0}\left(G_{0}^{1}-G_{0}^{0}\right) \leq C \mathcal{D}^{2} .
$$


Similarly, we observe by the definition of $\phi_{0}, Q_{W}^{0}>0$, and the fact that $\phi_{0}\left(u_{0}\right) \leq M$ that

$$
\left\|G_{0}^{0}\right\|_{L^{2}(S)}^{2} \leq C \int_{S} Q_{W}^{0}\left(G_{0}^{0}\right) \leq C M .
$$

We now start with the proof of (i). First, we observe

$$
\frac{1}{12} \int_{I} Q_{R}^{1}\left(\left(w_{s}^{\prime \prime}, \theta_{s}^{\prime}\right)-\left(w_{0}^{\prime \prime}, \theta_{0}^{\prime}\right)\right)=s^{2} \frac{1}{12} \int_{I} Q_{R}^{1}\left(\left(w_{1}^{\prime \prime}, \theta_{1}^{\prime}\right)-\left(w_{0}^{\prime \prime}, \theta_{0}^{\prime}\right)\right) \text {. }
$$

We will show that there exists $C>0$ independently of $s$ such that

$$
\int_{S} Q_{R}^{0}\left(G_{0}^{s}-G_{0}^{0}\right) \leq s^{2} \int_{S} Q_{R}^{0}\left(G_{0}^{1}-G_{0}^{0}\right)+C s^{2} \mathcal{D}^{3}+C s^{2} \mathcal{D}^{4}
$$

for $s \in[0,1]$. Then, recalling the definition of $\mathcal{D}_{0}$, (i) follows for the function $\Phi^{1}(t)=\sqrt{t^{2}+C t^{3}+C t^{4}}$. To show (A.4), we obtain by an elementary expansion

$$
G_{0}^{s}-G_{0}^{0}=s\left(G_{0}^{1}-G_{0}^{0}-(1-s) B_{\text {diff }}\right) .
$$

Then (2.11) yields

$$
\begin{aligned}
\int_{S} Q_{R}^{0}\left(G_{0}^{s}-G_{0}^{0}\right)= & s^{2} \int_{S} Q_{R}^{0}\left(G_{0}^{1}-G_{0}^{0}\right)+s^{2}(1-s) \int_{S} Q_{R}^{0}\left(B_{\text {diff }}\right) \\
& -2 s^{2}(1-s) \int_{S} C_{R}^{0}\left(G_{0}^{1}-G_{0}^{0}\right) B_{\text {diff }},
\end{aligned}
$$

from which we deduce (A.4), using (A.1), (A.2), and the Cauchy-Schwarz inequality.

We now show (ii) for the function $\Phi_{M}^{2}(t)=C \sqrt{M} t^{2}+C t^{3}+C t^{4}$ for some $C>0$. Due to convexity of $s \mapsto \int_{I} Q_{W}^{1}\left(w_{s}^{\prime \prime}, \theta_{s}^{\prime}\right)$, it suffices to show

$$
\begin{aligned}
& \int_{S} Q_{W}^{0}\left(G_{0}^{s}\right) \leq(1-s) \int_{S} Q_{W}^{0}\left(G_{0}^{0}\right)+s \int_{S} Q_{W}^{0}\left(G_{0}^{1}\right)+C \sqrt{M} s \mathcal{D}^{2} \\
& \quad+C s \mathcal{D}^{3}+C s^{2} \mathcal{D}^{4} .
\end{aligned}
$$

By $\left(\right.$ A.5) we have $G_{0}^{s}=(1-s) G_{0}^{0}+s G_{0}^{1}-s(1-s) B_{\text {diff }}$, and then an elementary expansion yields

$$
\begin{aligned}
\left(G_{0}^{s}\right)^{2}= & (1-s)\left(G_{0}^{0}\right)^{2}+s\left(G_{0}^{1}\right)^{2} \\
& -(1-s) s\left(G_{0}^{0}-G_{0}^{1}\right)^{2}-2 s(1-s)^{2} G_{0}^{0} B_{\text {diff }} \\
& -2 s^{2}(1-s) G_{0}^{1} B_{\text {diff }}+s^{2}(1-s)^{2} B_{\text {diff }}^{2} .
\end{aligned}
$$

Thus, by taking the integral and using the Cauchy-Schwarz inequality we get

$$
\begin{aligned}
\int_{S} Q_{W}^{0}\left(G_{0}^{s}\right) \leq & (1-s) \int_{S} Q_{W}^{0}\left(G_{0}^{0}\right)+s \int_{S} Q_{W}^{0}\left(G_{0}^{1}\right) \\
& +C s\left(\left\|G_{0}^{0}\right\|_{L^{2}(S)}+\left\|G_{0}^{1}\right\|_{L^{2}(S)}\right)\left\|B_{\mathrm{diff}}\right\|_{L^{2}(S)}+C s^{2}\left\|B_{\mathrm{diff}}\right\|_{L^{2}(S)}^{2} .
\end{aligned}
$$

By using $\left\|G_{0}^{1}\right\|_{L^{2}(S)} \leq\left\|G_{0}^{0}\right\|_{L^{2}(S)}+\left\|G_{0}^{1}-G_{0}^{0}\right\|_{L^{2}(S)},(\mathrm{A} .1),(\mathrm{A} .2)$, and (A.3) we get (A.6). This concludes the proof of (ii). 
We are now ready to prove the representation of the local slope stated in Lemma 4.3(i), which will help us to show that the local slope is weakly lower semicontinuous and a strong upper gradient.

Proof of Lemma 4.3(i). Let $\Phi^{1}$ and $\Phi_{M}^{2}$ be defined as in Lemma A.1 and note that $\lim _{t \rightarrow 0} \Phi^{1}(t) / t=1$ and $\lim _{t \rightarrow 0} \Phi_{M}^{2}(t) / t=0$. This and the property that $(a+b)^{+} \leq a^{+}+b^{+}$for every $a, b \in \mathbb{R}$ yields

$\left|\partial \phi_{0}\right|_{\mathcal{D}_{0}}(y, w, \theta)$

$$
\begin{aligned}
& =\lim _{(\tilde{y}, \tilde{w}, \tilde{\theta}) \rightarrow(y, w, \theta)} \frac{\left(\phi_{0}(y, w, \theta)-\phi_{0}(\tilde{y}, \tilde{w}, \tilde{\theta})\right)^{+}}{\mathcal{D}_{0}((y, w, \theta),(\tilde{y}, \tilde{w}, \tilde{\theta}))} \\
& \leq \lim _{(\tilde{y}, \tilde{w}, \tilde{\theta}) \rightarrow(y, w, \theta)} \frac{\left(\phi_{0}(y, w, \theta)-\phi_{0}(\tilde{y}, \tilde{w}, \tilde{\theta})-\Phi_{M}^{2}\left(\mathcal{D}_{0}((y, w, \theta),(\tilde{y}, \tilde{w}, \tilde{\theta}))\right)\right)^{+}}{\Phi^{1}\left(\mathcal{D}_{0}((y, w, \theta),(\tilde{y}, \tilde{w}, \tilde{\theta}))\right)} \\
& \leq \sup _{(y, w, \theta) \neq(\tilde{y}, \tilde{w}, \tilde{\theta}) \in \mathscr{S}^{1 D}} \frac{\left(\phi_{0}(y, w, \theta)-\phi_{0}(\tilde{y}, \tilde{w}, \tilde{\theta})-\Phi_{M}^{2}\left(\mathcal{D}_{0}((y, w, \theta),(\tilde{y}, \tilde{w}, \tilde{\theta}))\right)\right)^{+}}{\Phi^{1}\left(\mathcal{D}_{0}((y, w, \theta),(\tilde{y}, \tilde{w}, \tilde{\theta}))\right)} .
\end{aligned}
$$

To see the other inequality, we write $u_{0}=(y, w, \theta)$ and fix $u_{1}:=(\tilde{y}, \tilde{w}, \tilde{\theta})$ with $u_{0} \neq u_{1}$. Define $u_{s}:=(1-s) u_{0}+s u_{1}$. By Lemma A.1 we obtain the inequality

$$
\frac{\left(\phi_{0}\left(u_{0}\right)-\phi_{0}\left(u_{s}\right)\right)^{+}}{\mathcal{D}_{0}\left(u_{0}, u_{s}\right)} \geq \frac{\left(s \phi_{0}\left(u_{0}\right)-s \phi_{0}\left(u_{1}\right)-s \Phi_{M}^{2}\left(\mathcal{D}_{0}\left(u_{0}, u_{1}\right)\right)\right)^{+}}{s \Phi^{1}\left(\mathcal{D}_{0}\left(u_{0}, u_{1}\right)\right)} .
$$

In view of Theorem 4.2 (ii), $u_{s} \rightarrow u_{0}$ as $s \rightarrow 0$ with respect to the topology induced by $\mathcal{D}_{0}$, i.e., by letting $s \rightarrow 0$ the left-hand side is smaller or equal to $\left|\partial \phi_{0}\right|_{\mathcal{D}_{0}}\left(u_{0}\right)$. Taking the supremum on the right-hand side over all $u_{1} \in \mathscr{S}^{1 D}$, $u_{1} \neq u_{0}$, yields the lower bound for the local slope.

Similarly to Lemma 5.3, we construct a mutual recovery sequence as a key ingredient to show weak lower semicontinuity of slopes.

Lemma A.2. (Mutual recovery sequence in the one-dimensional setting) Let $\left(z_{k}\right)_{k} \subset \mathscr{S}^{1 D}$ be a sequence such that $z_{k} \rightarrow z$ and $u \in \mathscr{S}^{1 D}$. Then there exists a sequence $\left(u_{k}\right)_{k} \subset \mathscr{S}^{1 D}$ such that

(i) $\lim _{k \rightarrow \infty} \mathcal{D}_{0}\left(z_{k}, u_{k}\right)=\mathcal{D}_{0}(z, u)$,

(ii) $\phi_{0}(z)-\phi_{0}(u)=\lim _{k \rightarrow \infty}\left(\phi_{0}\left(z_{k}\right)-\phi_{0}\left(u_{k}\right)\right)$.

Proof. Let $z_{k}=\left(y_{k}, w_{k}, \theta_{k}\right)$ such that $z_{k} \rightarrow z=(y, w, \theta) \in \mathscr{S}^{1 D}$ and consider $u=(\tilde{y}, \tilde{w}, \tilde{\theta}) \in \mathscr{S}^{1 D}$. We define the mutual recovery sequence by

$$
u_{k}:=\left(\tilde{y}_{k}, \tilde{w}_{k}, \tilde{\theta}_{k}\right)=\left(y_{k}+\tilde{y}-y, w_{k}+\tilde{w}-w, \theta_{k}+\tilde{\theta}-\theta\right) .
$$

Then, by the compact embedding $W^{1,2}(I) \subset \subset L^{4}(I)$, we have

$$
\left|w_{k}^{\prime}\right|^{2}-\left|\tilde{w}_{k}^{\prime}\right|^{2} \rightarrow\left|w^{\prime}\right|^{2}-\left|\tilde{w}^{\prime}\right|^{2} \text { in } L^{2}(I) .
$$

Moreover, by construction it holds that $\partial_{1}\left(y_{k}\right)_{1}-\partial_{1}\left(\tilde{y}_{k}\right)_{1}=\partial_{1} y_{1}-\partial_{1} \tilde{y}_{1}, w_{k}^{\prime \prime}-$ $\tilde{w}_{k}^{\prime \prime}=w^{\prime \prime}-\tilde{w}^{\prime \prime}$, and $\theta_{k}^{\prime}-\tilde{\theta}_{k}^{\prime}=\theta^{\prime}-\tilde{\theta}^{\prime}$. This implies (i) since 


$$
\begin{aligned}
\lim _{k \rightarrow \infty} \mathcal{D}_{0}^{2}\left(\left(y_{k}, w_{k}, \theta_{k}\right),\left(\tilde{y}_{k}, \tilde{w}_{k}, \tilde{\theta}_{k}\right)\right)= & \int_{S} Q_{R}^{0}\left(\partial_{1} y_{1}-\partial_{1} \tilde{y}_{1}+\frac{\left|w^{\prime}\right|^{2}}{2}-\frac{\left|\tilde{w}^{\prime}\right|^{2}}{2}\right) \\
& +\frac{1}{12} \int_{I} Q_{R}^{1}\left(w^{\prime \prime}-\tilde{w}^{\prime \prime}, \theta^{\prime}-\tilde{\theta}^{\prime}\right) .
\end{aligned}
$$

We now address (ii). We only have weak convergence of $\left(\partial_{1}\left(y_{k}\right)_{1}+\frac{1}{2}\left|w_{k}^{\prime}\right|^{2}\right)_{k}$ in $L^{2}(S)$, but strong convergence of $\left(\partial_{1}\left(\tilde{y}_{k}\right)_{1}+\frac{1}{2}\left|\tilde{w}_{k}^{\prime}\right|^{2}-\partial_{1}\left(y_{k}\right)_{1}+\frac{1}{2}\left|w_{k}^{\prime}\right|^{2}\right)_{k}$ in $L^{2}(S)$. Thus, by adapting the arguments in Step 3 of the proof of Lemma 5.3, in particular using (5.5), we find as $k \rightarrow \infty$

$$
\begin{gathered}
\int_{S}\left(Q_{W}^{0}\left(\partial_{1}\left(y_{k}\right)_{1}+\frac{\left|w_{k}^{\prime}\right|^{2}}{2}\right)-Q_{W}^{0}\left(\partial_{1}\left(\tilde{y}_{k}\right)_{1}+\frac{\left|\tilde{w}_{k}^{\prime}\right|^{2}}{2}\right)\right) \\
\rightarrow \int_{S}\left(Q_{W}^{0}\left(\partial_{1} y_{1}+\frac{\left|w^{\prime}\right|^{2}}{2}\right)-Q_{W}^{0}\left(\partial_{1} \tilde{y}_{1}+\frac{\left|\tilde{w}^{\prime}\right|^{2}}{2}\right)\right) .
\end{gathered}
$$

The observation that

$$
\int_{I} Q_{W}^{1}\left(w_{k}^{\prime \prime}-\tilde{w}_{k}^{\prime \prime}, \theta_{k}^{\prime}-\tilde{\theta}_{k}^{\prime}\right)=\int_{I} Q_{W}^{1}\left(w^{\prime \prime}-\tilde{w}^{\prime \prime}, \theta^{\prime}-\tilde{\theta}^{\prime}\right)
$$

for every $k \in \mathbb{N}$ concludes the proof of (ii).

We now proceed with the proof of Lemma 4.3(ii),(iii).

Proof of Lemma 4.3(ii), (iii). We first show (ii). As the local slope is a weak upper gradient in the sense of Definition [4, Definition 1.2.2] by [4, Theorem 1.2.5], we only need to show that for an absolutely continuous curve $z:(a, b) \rightarrow \mathscr{S}^{1 D}$ satisfying $\left|\partial \phi_{0}\right|_{\mathcal{D}_{0}}(z)\left|z^{\prime}\right|_{\mathcal{D}_{0}} \in L^{1}(a, b)$ the curve $\phi_{0} \circ z$ is absolutely continuous. It is not restrictive to assume that $(a, b)$ is a bounded interval and that the curve $z$ is extended by continuity to $[a, b]$. Thus, $\mathscr{S}_{z}^{1 D}:=z([a, b])$ is compact and we define $\operatorname{diam}\left(\mathscr{S}_{z}^{1 D}\right):=\sup _{s, t \in[a, b]} \mathcal{D}_{0}(z(s), z(t))<+\infty$. Thus, by Lemma $4.2(\mathrm{ii})$ we find an $M>0$ such that $\phi_{0}(z(s)) \leq M$ for every $s \in[a, b]$. Since $\phi_{0}$ is $\mathcal{D}_{0}$-lower semicontinuous by Lemma 4.2 (ii) and (iv), the global slope

$$
I_{\phi_{0}}(v):=\sup _{v \neq w \in \mathscr{S}_{z}^{1 D}} \frac{\left(\phi_{0}(v)-\phi_{0}(w)\right)^{+}}{\mathcal{D}_{0}(v, w)}
$$

is a strong upper gradient with respect to $\mathscr{S}_{z}^{1 D}$ by [4, Theorem 1.2.5]. Thus, it holds for all $a<s \leq t<b$ that

$$
\left|\phi_{0}(z(t))-\phi_{0}(z(s))\right| \leq \int_{s}^{t} I_{\phi_{0}}(z(r))\left|z^{\prime}\right|_{\mathcal{D}_{0}}(r) \mathrm{d} r
$$

see Definition 3.1(i). The claim follows once we bound $I_{\phi_{0}}(z)\left|z^{\prime}\right|_{\mathcal{D}_{0}}$ with an integrable function. To this end, we define the constants $C_{1}:=\sup _{t \in\left[0, \operatorname{diam}\left(\mathscr{S}_{z}^{1 D}\right)\right]} \frac{\Phi^{1}(t)}{t}<+\infty \quad$ and $\quad C_{2}:=\sup _{t \in\left[0, \operatorname{diam}\left(\mathscr{S}_{z}^{1 D}\right)\right]} \frac{\Phi_{M}^{2}(t)}{t}<+\infty$, 
where $\Phi^{1}$ and $\Phi_{M}^{2}$ are given in Lemma 4.3(i). Hence, for every $v \in \mathscr{S}_{z}^{1 D}$ it holds that

$$
\begin{aligned}
I_{\phi_{0}}(v) \leq & \sup _{v \neq w \in \mathscr{S}_{z}^{1 D}} \frac{\left(\phi_{0}(v)-\phi_{0}(w)-\Phi_{M}^{2}\left(\mathcal{D}_{0}(v, w)\right)\right)^{+}}{\Phi^{1}\left(\mathcal{D}_{0}(v, w)\right)} \frac{\Phi^{1}\left(\mathcal{D}_{0}(v, w)\right)}{\mathcal{D}_{0}(v, w)} \\
& +\sup _{v \neq w \in \mathscr{S}_{z}^{1 D}} \frac{\left(\Phi_{M}^{2}\left(\mathcal{D}_{0}(v, w)\right)\right)^{+}}{\mathcal{D}_{0}(v, w)} \\
\leq & C_{1}\left|\partial \phi_{0}\right|_{\mathcal{D}_{0}}(v)+C_{2} .
\end{aligned}
$$

By using the assumption that $\left|\partial \phi_{0}\right|_{\mathcal{D}_{0}}(z)\left|z^{\prime}\right|_{\mathcal{D}_{0}} \in L^{1}(a, b)$ we get the absolute continuity.

We now show (iii). Let $\left(y_{k}, w_{k}, \theta_{k}\right)_{k} \subset \mathscr{S}^{1 D}$ be such that $\left(y_{k}, w_{k}, \theta_{k}\right) \rightarrow$ $(y, w, \theta)$ for some $(y, w, \theta) \in \mathscr{S}^{1 D}$. We let $\varepsilon>0$ and define $z_{k}:=\left(y_{k}, w_{k}, \theta_{k}\right)$, $z:=(y, w, \theta)$, and $M:=\phi_{0}(z)$. By Lemma $4.3(\mathrm{i})$ there exists $u \in \mathscr{S}^{1 D}$ such that

$$
\left|\partial \phi_{0}\right|_{\mathcal{D}_{0}}(z) \leq \frac{\left(\phi_{0}(z)-\phi_{0}(u)-\Phi_{M}^{2}\left(\mathcal{D}_{0}(z, u)\right)\right)^{+}}{\Phi^{1}\left(\mathcal{D}_{0}(z, u)\right)}+\varepsilon
$$

Let $\left(u_{k}\right)_{k} \subset \mathscr{S}^{1 D}$ be the sequence given by Lemma A.2. Since $\Phi^{1}$ and $\Phi_{M}^{2}$ are continuous, we obtain

$$
\begin{aligned}
\left|\partial \phi_{0}\right|_{\mathcal{D}_{0}}(z) & \leq \liminf _{k \rightarrow \infty} \frac{\left(\phi_{0}\left(z_{k}\right)-\phi_{0}\left(u_{k}\right)-\Phi_{M}^{2}\left(\mathcal{D}_{0}\left(z_{k}, u_{k}\right)\right)\right)^{+}}{\Phi^{1}\left(\mathcal{D}_{0}\left(z_{k}, u_{k}\right)\right)}+\varepsilon \\
& \leq \liminf _{k \rightarrow \infty}\left|\partial \phi_{0}\right|_{\mathcal{D}_{0}}\left(z_{k}\right)+\varepsilon
\end{aligned}
$$

where the last step follows again by Lemma 4.3(i). The statement follows by sending $\varepsilon \rightarrow 0$.

We close this section by proving the fine representation for the local slope given in Lemma 6.3.

Proof of Lemma 6.3. To simplify the notation, we will write $(\tilde{y}, \tilde{w}, \tilde{\theta}) \rightarrow(y, w, \theta)$ instead of $\mathcal{D}_{0}((\tilde{y}, \tilde{w}, \tilde{\theta}),(y, w, \theta)) \rightarrow 0$. Recall the definitions in (6.1) and (6.3). The embedding $W^{1,2}(I) \subset \subset L^{4}(I)$ yields $\|G(y, w, \theta)-G(\tilde{y}, \tilde{w}, \tilde{\theta})\|_{L^{2}\left(S ; \mathbb{R}^{3}\right)} \rightarrow 0$ as $(\tilde{y}, \tilde{w}, \tilde{\theta}) \rightarrow(y, w, \theta)$. Then, by the positivity of $\bar{Q}_{R}$ we get

$$
\frac{\int_{S} \bar{Q}_{W}(G(y, w, \theta)-G(\tilde{y}, \tilde{w}, \tilde{\theta}))}{\left(\int_{S} \bar{Q}_{R}(G(y, w, \theta)-G(\tilde{y}, \tilde{w}, \tilde{\theta}))\right)^{1 / 2}} \leq C \frac{\|G(y, w, \theta)-G(\tilde{y}, \tilde{w}, \tilde{\theta})\|_{L^{2}\left(S ; \mathbb{R}^{3}\right)}^{2}}{\|G(y, w, \theta)-G(\tilde{y}, \tilde{w}, \tilde{\theta})\|_{L^{2}\left(S ; \mathbb{R}^{3}\right)}} \rightarrow 0
$$


This along with the expansion (5.5) and Lemma 6.2 yields

$$
\begin{aligned}
\left|\partial \phi_{0}\right|_{\mathcal{D}_{0}}(y, w, \theta) & =\limsup _{(\tilde{y}, \tilde{w}, \tilde{\theta}) \rightarrow(y, w, \theta)} \frac{\left(\int_{S} \frac{1}{2} \bar{Q}_{W}(G(y, w, \theta))-\frac{1}{2} \bar{Q}_{W}(G(\tilde{y}, \tilde{w}, \tilde{\theta}))\right)^{+}}{\left(\int_{S} \bar{Q}_{R}(G(y, w, \theta)-G(\tilde{y}, \tilde{w}, \tilde{\theta}))\right)^{1 / 2}} \\
= & \limsup _{(\tilde{y}, \tilde{w}, \tilde{\theta}) \rightarrow(y, w, \theta)} \frac{\left(\int_{S} \overline{\mathbb{C}}_{W}[G(y, w, \theta), G(y, w, \theta)-G(\tilde{y}, \tilde{w}, \tilde{\theta})]\right)^{+}}{\left(\int_{S} \bar{Q}_{R}(G(y, w, \theta)-G(\tilde{y}, \tilde{w}, \tilde{\theta}))\right)^{1 / 2}} \\
= & \limsup _{(\tilde{y}, \tilde{w}, \tilde{\theta}) \rightarrow(y, w, \theta)} \frac{\left(\int_{S} \overline{\mathbb{C}}_{W}\left[G(y, w, \theta), H(y-\tilde{y}, w-\tilde{w}, \theta-\tilde{\theta} \mid w)-\frac{1}{2}\left(\left(w^{\prime}-\tilde{w}^{\prime}\right)^{2}, 0,0\right)\right]\right)^{+}}{\left(\int_{S} \bar{Q}_{R}(G(y, w, \theta)-G(\tilde{y}, \tilde{w}, \tilde{\theta}))\right)^{1 / 2}},
\end{aligned}
$$

where the last equality follows from (6.2). Due to (4.1), (6.2), and Lemma 6.2, we find that

$$
\begin{aligned}
& \frac{\int_{S} \bar{Q}_{R}(G(y, w, \theta)-G(\tilde{y}, \tilde{w}, \tilde{\theta}))}{\int_{S} \bar{Q}_{R}(H(y-\tilde{y}, w-\tilde{w}, \theta-\tilde{\theta} \mid w))} \rightarrow 1, \\
& \quad \frac{\int_{S}\left(w^{\prime}-\tilde{w}^{\prime}\right)^{2}}{\left(\int_{S} \bar{Q}_{R}(G(y, w, \theta)-G(\tilde{y}, \tilde{w}, \tilde{\theta}))\right)^{1 / 2}} \rightarrow 0
\end{aligned}
$$

as $(\tilde{y}, \tilde{w}, \tilde{\theta}) \rightarrow(y, w, \theta)$. Thus, we obtain

$$
\left|\partial \phi_{0}\right|_{\mathcal{D}_{0}}(y, w, \theta)=\limsup _{(\tilde{y}, \tilde{w}, \tilde{\theta}) \rightarrow(y, w, \theta)} \frac{\left(\int_{S} \overline{\mathbb{C}}_{W}[G(y, w, \theta), H(y-\tilde{y}, w-\tilde{w}, \theta-\tilde{\theta} \mid w)]\right)^{+}}{\left(\int_{S} \bar{Q}_{R}(H(y-\tilde{y}, w-\tilde{w}, \theta-\tilde{\theta} \mid w))\right)^{1 / 2}} .
$$

We introduce the space of test functions $\mathcal{P}:=B N_{(0,0)}\left(S, \mathbb{R}^{2}\right) \times W_{0}^{2,2}(I) \times$ $W_{0}^{1,2}(I)$. Since the operator $H$ is linear, we can simplify this expression by substitution with sequences that converge to 0 . Moreover, as the enumerator and denominator are positively homogeneous of degree one, we derive the representation

$$
\left|\partial \phi_{0}\right|_{\mathcal{D}_{0}}(y, w, \theta)=\sup _{0 \neq(\hat{y}, \hat{w}, \hat{\theta}) \in \mathcal{P}} \frac{\left(\int_{S} \overline{\mathbb{C}}_{W}[G(y, w, \theta), H(\hat{y}, \hat{w}, \hat{\theta} \mid w)]\right)^{+}}{\left\|\sqrt{\overline{\mathbb{C}}_{R}} H(\hat{y}, \hat{w}, \hat{\theta} \mid w)\right\|_{L^{2}\left(S ; \mathbb{R}^{3}\right)}},
$$

where we have used the definition of $\sqrt{\overline{\mathbb{C}}_{R}}$. We now want to show that the supremum is attained by considering the minimization problem

$$
\min _{(\bar{y}, \bar{w}, \bar{\theta}) \in \mathcal{P}} \mathcal{F}(\bar{y}, \bar{w}, \bar{\theta})
$$

where

$$
\mathcal{F}(\bar{y}, \bar{w}, \bar{\theta}):=\frac{1}{2} \int_{S}\left|\sqrt{\overline{\mathbb{C}}_{R}} H(\bar{y}, \bar{w}, \bar{\theta} \mid w)\right|^{2}-\int_{S} \overline{\mathbb{C}}_{W}[G(y, w, \theta), H(\bar{y}, \bar{w}, \bar{\theta} \mid w)] .
$$

The existence of a solution can be guaranteed by the direct method of the calculus of variations. The functional $\mathcal{F}$ is weakly lower semicontinuous as $\sqrt{\overline{\mathbb{C}}_{R}}$ and $H$ are linear operators and $|\cdot|^{2}$ is convex. To show coercivity, we consider a constant $C>0$ such that $\mathcal{F}(\bar{y}, \bar{w}, \bar{\theta}) \leq C$. Since $\overline{\mathbb{C}}_{R}[H(\bar{y}, \bar{w}, \bar{\theta} \mid w), H(\bar{y}, \bar{w}, \bar{\theta} \mid w)] \geq$ $c|H(\bar{y}, \bar{w}, \bar{\theta} \mid w)|^{2}$ by the positivity of $\bar{Q}_{R}$, we obtain by Cauchy-Schwarz

$$
\|H(\bar{y}, \bar{w}, \bar{\theta} \mid w)\|_{L^{2}\left(S ; \mathbb{R}^{3}\right)} \leq C,
$$


where $C$ depends on $y, w$, and $\theta$. Arguing similarly to the proof of Lemma 4.2(ii), we find that $\|\bar{w}\|_{W^{2,2}(I)} \leq C,\|\bar{\theta}\|_{W^{1,2}(I)} \leq C$, and $\|\bar{y}\|_{W^{1,2}\left(S ; \mathbb{R}^{2}\right)} \leq C$. Thus, there exists a unique minimizer $\left(y_{*}, w_{*}, \theta_{*}\right) \in \mathcal{P}$. By computing the EulerLagrange equations, we observe that the minimum satisfies

$$
\begin{gathered}
\int_{S} \sqrt{\overline{\mathbb{C}}_{R}} H\left(y_{*}, w_{*}, \theta_{*} \mid w\right) \cdot \sqrt{\overline{\mathbb{C}}_{R}} H\left(\phi_{y}, \phi_{w}, \phi_{\theta} \mid w\right) \\
-\int_{S} \overline{\mathbb{C}}_{W}\left[G(y, w, \theta), H\left(\phi_{y}, \phi_{w}, \phi_{\theta} \mid w\right)\right]
\end{gathered}
$$

for all $\left(\phi_{y}, \phi_{w}, \phi_{\theta}\right) \in \mathcal{P}$. This equation can also be formulated as

$$
\int_{S} \mathcal{L}(y, w, \theta) \cdot H\left(\phi_{y}, \phi_{w}, \phi_{\theta} \mid w\right)=0
$$

for all $\left(\phi_{y}, \phi_{w}, \phi_{\theta}\right) \in \mathcal{P}$, where we define the operator $\mathcal{L}$ by

$$
\mathcal{L}(y, w, \theta):=\overline{\mathbb{C}}_{R} H\left(y_{*}, w_{*}, \theta_{*} \mid w\right)-\overline{\mathbb{C}}_{W} G(y, w, \theta) .
$$

By (6.1) and the regularity of the functions, we find $\mathcal{L}(y, w, \theta) \in L^{2}\left(S ; \mathbb{R}^{3}\right)$. By (A.7), (A.8), and the definition of $\mathcal{L}$ we then get

$$
\begin{aligned}
\left|\partial \phi_{0}\right|_{\mathcal{D}_{0}}(y, w, \theta) & \geq \frac{\int_{S}\left(\overline{\mathbb{C}}_{W} G(y, w, \theta)+\mathcal{L}(y, w, \theta)\right) \cdot H\left(y_{*}, w_{*}, \theta_{*} \mid w\right)}{\left\|\sqrt{\overline{\mathbb{C}}_{R}} H\left(y_{*}, w_{*}, \theta_{*} \mid w\right)\right\|_{L^{2}\left(S ; \mathbb{R}^{3}\right)}} \\
& =\frac{\int_{S} \sqrt{\overline{\mathbb{C}}_{R}}-1\left(\overline{\mathbb{C}}_{W} G(y, w, \theta)+\mathcal{L}(y, w, \theta)\right) \cdot \sqrt{\overline{\mathbb{C}}_{R}} H\left(y_{*}, w_{*}, \theta_{*} \mid w\right)}{\left\|\sqrt{\overline{\mathbb{C}}_{R}} H\left(y_{*}, w_{*}, \theta_{*} \mid w\right)\right\|_{L^{2}\left(S ; \mathbb{R}^{3}\right)}} \\
& =\left\|\sqrt{\overline{\mathbb{C}}_{R}} H\left(y_{*}, w_{*}, \theta_{*} \mid w\right)\right\|_{L^{2}\left(S ; \mathbb{R}^{3}\right)} \\
& =\left\|\sqrt{\overline{\mathbb{C}}_{R}-1}\left(\overline{\mathbb{C}}_{W} G(y, w, \theta)+\mathcal{L}(y, w, \theta)\right)\right\|_{L^{2}\left(S ; \mathbb{R}^{3}\right)} \cdot
\end{aligned}
$$

On the other hand, by a similar argument, in view of (A.7) and (A.8), we find

$$
\begin{aligned}
\left|\partial \phi_{0}\right|_{\mathcal{D}_{0}}(y, w, \theta) & =\sup _{0 \neq(\hat{y}, \hat{w}, \hat{\theta}) \in \mathcal{P}} \frac{\int_{S}\left(\overline{\mathbb{C}}_{W} G(y, w, \theta)+\mathcal{L}(y, w, \theta)\right) \cdot H(\hat{y}, \hat{w}, \hat{\theta} \mid w)}{\left\|\sqrt{\overline{\mathbb{C}}}{ }_{R} H(\hat{y}, \hat{w}, \hat{\theta} \mid w)\right\|_{L^{2}\left(S ; \mathbb{R}^{3}\right)}} \\
& \leq\left\|{\sqrt{\overline{\mathbb{C}}_{R}}}^{-1}\left(\overline{\mathbb{C}}_{W} G(y, w, \theta)+\mathcal{L}(y, w, \theta)\right)\right\|_{L^{2}\left(S ; \mathbb{R}^{3}\right)},
\end{aligned}
$$

where we again distributed $\sqrt{\overline{\mathbb{C}}_{R}}$ suitably to the two terms and used the Cauchy-Schwarz inequality. This concludes the proof.

\section{References}

[1] Abels, H., Mora, M.G., Müller, S.: The time-dependent von Kármán plate equation as a limit of 3d nonlinear elasticity. Calc. Var. PDE 41, 241-259 (2011)

[2] Acerbi, E., Buttazzo, G., Percivale, D.: A variational definition for the strain energy of an elastic string. J. Elasticity 25, 137-148 (1991) 
[3] Adams, R.A., Fournier, J.J.F.: Sobolev Spaces, 2nd edn. Elsevier, Amsterdam (2003)

[4] Ambrosio, L., Gigli, N., Savaré, G.: Gradient Flows in Metric Spaces and in the Space of Probability Measures. Lectures Math. ETH Zürich, Birkhäuser, Basel (2005)

[5] Antman, S.S.: Physically unacceptable viscous stresses. Z. Angew. Math. Phys. 49, 980-988 (1998)

[6] Antman, S.S.: Nonlinear Problems of Elasticity. Springer, New York (2004)

[7] Ball, J.M.: Convexity conditions and existence theorems in nonlinear elasticity. Arch. Ration. Mech. Anal. 63, 337-403 (1977)

[8] Bermudez, A., Viaño, J.M.: Une justification des équations de la thermoélasticité des poutres à section variable par des méthodes asymptotiques. RAIRO. Analyse Numérique 18, 347-376 (1984)

[9] Bock, I.: On von Kármán equations for viscoelastic plates. J. Comput. Appl. Math. 63, 277-282 (1995)

[10] Bock, I., Jarušek, J.: Solvability of dynamic contact problems for elastic von Kármán plates. SIAM J. Math. Anal. 41, 37-45 (2009)

[11] Bock, I., Jarušek, J., Šilhavý, M.: On the solutions of a dynamic contact problem for a thermoelastic von Kármán plate. Nonlinear Anal. Real World Appl. 32, 111-135 (2016)

[12] Ciarlet, P.G.: Mathematical elasticity. Vol II: Theory of plates volume 27 of Studies in Mathematics and its Applications. North-Holland Publishing Co., Amsterdam (1997)

[13] Dal Maso, G.: An Introduction to Г-Convergence. Birkhäuser, Boston, Basel, Berlin (1993)

[14] De Giorgi, E., Marino, A., Tosques, M.: Problems of evolution in metric spaces and maximal decreasing curve. Att. Accad. Naz. Lincei Rend. Cl. Sci. Fis. Mat. Natur. 68, 180-187 (1980)

[15] Demoulini, S.: Weak solutions for a class of nonlinear systems of viscoelasticity. Arch. Ration. Mech. Anal. 155, 299-334 (2000)

[16] Fosdick, R., Fried, E.: The Mechanics of Ribbons and Möbius Bands. Springer, Netherlands (2016)

[17] Freddi, L., Mora, M.G., Paroni, R.: Nonlinear thin-walled beams with a rectangular cross-section - Part I. Math. Models Methods Appl. Sci. 22 (2012), 1150016 (34 pp)

[18] Freddi, L., Mora, M.G., Paroni, R.: Nonlinear thin-walled beams with a rectangular cross-section - Part II. Math. Models Methods Appl. Sci. 23, 743-775 (2013) 
[19] Freddi, L., Hornung, P., Mora, M.G., Paroni, R.: A corrected Sadowsky functional for inextensible elastic ribbons. J. Elasticity 123, 125-136 (2016)

[20] Freddi, L., Hornung, P., Mora, M.G., Paroni, R.: One-dimensional von Kármán models for elastic ribbons. Meccanica 53, 659-670 (2018)

[21] Friedrich, M., Kružík, M.: On the passage from nonlinear to linearized viscoelasticity. SIAM J. Math. Anal. 50, 4426-4456 (2018)

[22] Friedrich, M., Kružík, M.: Derivation of von Kármán Plate Theory in the Framework of Three-Dimensional Viscoelasticity. Arch. Ration. Mech. Anal. 238, 489$540(2020)$

[23] Friedrich, M., Kružík, M., Valdman, J.: Numerical approximation of von Kármán viscoelastic plates. Disc. Cont. Dyn. Syst. 14, 299-319 (2021)

[24] Friesecke, G., James, R.D., Müller, S.: A theorem on geometric rigidity and the derivation of nonlinear plate theory from three-dimensional elasticity. Commun. Pure Appl. Math. 55, 1461-1506 (2002)

[25] Friesecke, G., James, R.D., Müller, S.: A hierarchy of plate models derived from nonlinear elasticity by Gamma-Convergence. Arch. Ration. Mech. Anal. 180, 183-236 (2006)

[26] Hinz, D.F., Fried, E.: Translation of Michael Sadowsky's paper "An elementary proof for the existence of a developable Möbius band and the attribution of the geometric problem to a variational problem". J. Elasticity 119, 3-6 (2015)

[27] Lecumberry, M., Müller, S.: Stability of slender bodies under compression and validity of the von Kármán theory. Arch. Ration. Mech. Anal. 193, 255-310 (2009)

[28] Lewicka, M., Mucha, P.B.: A local existence result for system of viscoelasticity with physical viscosity. Evolut. Equ. Control Theory 2, 337-353 (2013)

[29] Mielke, A.: On evolutionary $\Gamma$-convergence for gradient systems (ch. 3). In: Muntean, A. Rademacher, J., Zagaris, A. (eds). Macroscopic and Large Scale Phenomena: Coarse Graining, Mean Field Limits and Ergodicity. Lecture Notes in Applied Math. Mechanics Vol. 3, 187-249. Springer, 2016. Proc. of Summer School in Twente University, June (2012)

[30] Mielke, A., Ortner, C., Şengül, Y.: An approach to nonlinear viscoelasticity via metric gradient flows. SIAM J. Math. Anal. 46, 1317-1347 (2014)

[31] Mielke, A., Roubíček, T.: Thermoviscoelasticity in Kelvin-Voigt rheology at large strains. Arch. Ration. Mech. Anal. 238, 1-45 (2020)

[32] Mora, M.G., Müller, S.: Derivation of the nonlinear bending-torsion theory for inextensible rods by $\Gamma$-convergence. Calc. Var. PDE 18, 287-305 (2003)

[33] Mora, M.G., Müller, S.: A nonlinear model for inextensible rods as a low energy $\Gamma$-limit of three-dimensional nonlinear elasticity. Ann. Inst. H. Poincaré Anal. Non Linéaire 21, 271-293 (2004) 
[34] Ortner, C.: Two Variational Techniques for the Approximation of Curves of Maximal Slope. Technical report NA05/10, Oxford University Computing Laboratory, Oxford, UK, (2005)

[35] Park, J.Y., Kang, J.R.: Uniform decay of solutions for von Karman equations of dynamic viscoelasticity with memory. Acta Appl. Math. 110, 1461-1474 (2010)

[36] Sandier, E., Serfaty, S.: Gamma-convergence of gradient flows with applications to Ginzburg-Landau. Commun. Pure Appl. Math. 57, 1627-1672 (2004)

[37] Serfaty, S.: Gamma-convergence of gradient flows on Hilbert and metric spaces and applications. Discrete Contin. Dyn. Syst. Ser. A 31, 1427-1451 (2011)

[38] Todres, R.E.: Translation of W. Wunderlich's "On a developable Möbius band". J. Elasticity 119, 23-34 (2015)

[39] Toupin, R.A.: Elastic materials with couple stresses. Arch. Ration. Mech. Anal. 11, 385-414 (1962)

[40] Toupin, R.A.: Theory of elasticity with couple stress. Arch. Ration. Mech. Anal. 17, 85-112 (1964)

[41] von Kármán, T.: Festigkeitsprobleme im Maschinenbau in Encyclopädie der Mathematischen Wissenschaften. vol. IV/4, Leipzig, (1910), 311-385

Manuel Friedrich and Lennart Machill

Applied Mathematics

Universität Münster

Einsteinstr. 62

48149 Münster

Germany

e-mail: lennart.machill@uni-muenster.de

Manuel Friedrich

e-mail: manuel.friedrich@uni-muenster.de

Received: 6 September 2021.

Accepted: 7 December 2021. 